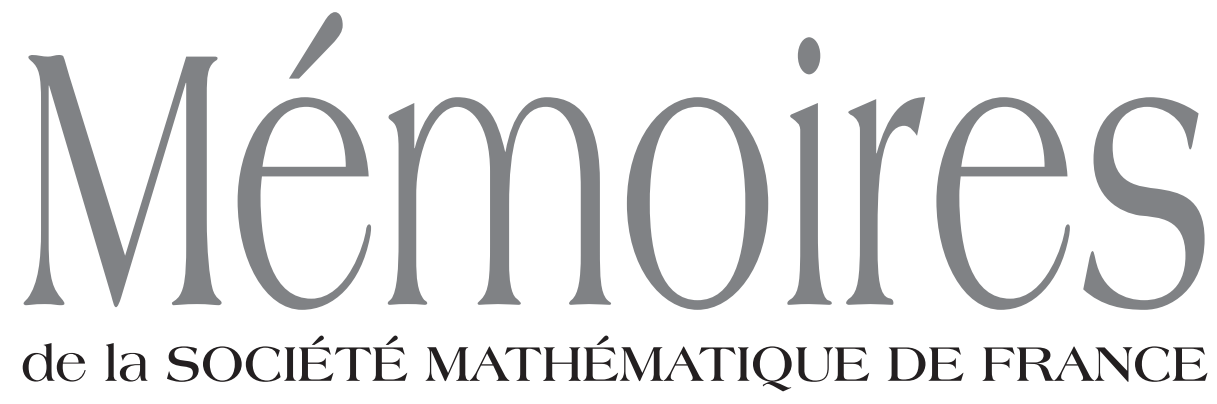

Numéro 122 ENTROPY OF MEROMORPHIC MAPS Nouvelle série AND DYNAMICS OF BIRATIONAL MAPS

Henry DE THÉLIN \& Gabriel VIGNY

$\begin{array}{llll}2 & 0 & 1 & 0\end{array}$

SOCIÉTÉ MATHÉMATIQUE DE FRANCE

Publié avec le concours du Centre National de la Recherche Scientifique 


\title{
Comité de rédaction
}

\author{
Jean BARGE \\ Emmanuel BREUILLARD \\ Gérard BESSON \\ Antoine CHAMBERT-LOIR \\ Jean-François DAT \\ Raphaël KRIKORIAN (dir.) \\ Charles FAVRE \\ Daniel HUYBRECHTS \\ Yves LE JAN \\ Laure SAINT-RAYMOND \\ Wilhem SCHLAG
}

\section{Diffusion}

Maison de la SMF

Case 916 - Luminy

13288 Marseille Cedex 9

France

smf@smf .univ-mrs.fr
Hindustan Book Agency

O-131, The Shopping Mall

Arjun Marg, DLF Phase 1

Gurgaon 122002, Haryana

Inde
AMS

P.O. Box 6248

Providence RI 02940

USA

WwW . ams . org

\section{Tarifs}

Vente au numéro : $28 €(\$ 42)$

Abonnement Europe : $255 €$, hors Europe : $290 €(\$ 435)$

Des conditions spéciales sont accordées aux membres de la SMF.

\section{Secrétariat : Nathalie Christiaën}

Mémoires de la SMF

Société Mathématique de France

Institut Henri Poincaré, 11, rue Pierre et Marie Curie

75231 Paris Cedex 05, France

Tél : (33) 0144276799 • Fax : (33) 0140469096

revues@smf.ens.fr • http://smf.emath.fr/

(C) Société Mathématique de France 2010

Tous droits réservés (article L 122-4 du Code de la propriété intellectuelle). Toute représentation ou reproduction intégrale ou partielle faite sans le consentement de l'éditeur est illicite. Cette représentation ou reproduction par quelque procédé que ce soit constituerait une contrefaçon sanctionnée par les articles L 335-2 et suivants du CPI.

ISSN 0249-633-X

ISBN 978-85629-302-7

Directrice de la publication : Aline BONAMI 


\section{ENTROPY OF MEROMORPHIC MAPS AND DYNAMICS OF BIRATIONAL MAPS}

Henry De Thélin

Gabriel Vigny

Société Mathématique de France 2010

Publié avec le concours du Centre National de la Recherche Scientifique 


\section{Henry De Thélin}

Laboratoire Analyse, Géométrie et Applications, UMR 7539, Institut Galilée, Université Paris 13, 99 av. J.-B. Clément, 93430 Villetaneuse, France.

E-mail : dethelin@math.univ-paris13.fr

Gabriel Vigny

LAMFA - UMR 6140, U. P. J. V. 33, rue Saint-Leu, 80039 Amiens, France.

E-mail : gabriel.vigny@u-picardie.fr

2000 Mathematics Subject Classification. - 37Fxx, 32H04, 32Uxx, 37A35, 37Dxx.

Key words and phrases. - Complex dynamics, meromorphic maps, super-potentials, currents, entropy, hyperbolic measure. 


\title{
ENTROPY OF MEROMORPHIC MAPS AND DYNAMICS OF BIRATIONAL MAPS
}

\author{
Henry De Thélin, Gabriel Vigny
}

\begin{abstract}
We study the dynamics of meromorphic maps for a compact Kähler manifold $X$. More precisely, we give a simple criterion that allows us to produce a measure of maximal entropy. We can apply this result to bound the Lyapunov exponents.

Then, we study the particular case of a family of generic birational maps of $\mathbb{P}^{k}$ for which we construct the Green currents and the equilibrium measure. We use for that the theory of super-potentials. We show that the measure is mixing and gives no mass to pluripolar sets. Using the criterion we get that the measure is of maximal entropy. It implies finally that the measure is hyperbolic.
\end{abstract}

\section{Résumé (Entropie des applications méromorphes et dynamique des applications bira- tionnelles)}

On étudie la dynamique des applications méromorphes sur les variétés kählériennes compactes. Plus précisément, on donne un critère simple qui permet de produire des mesures d'entropie maximale. On peut appliquer ce résultat pour borner les exposants de Lyapounov.

Ensuite, on étudie le cas particulier d'une famille générique d'applications birationnelles de $\mathbb{P}^{k}$ pour laquelle on construit les courants de Green et la mesure d'équilibre. On utilise pour cela la théorie des super-potentiels. On montre que la mesure est mélangeante et qu'elle n'a pas de masse sur les ensembles pluripolaires. En utilisant le critère on obtient que la mesure est d'entropie maximale. Cela implique finalement que la mesure est hyperbolique. 



\section{CONTENTS}

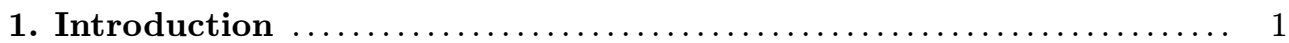

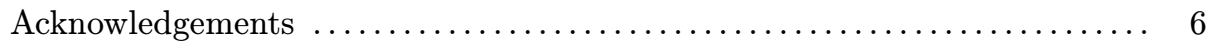

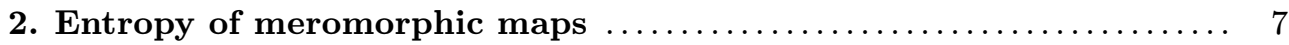

2.1. Push-forward of measures by meromorphic maps $\ldots \ldots \ldots \ldots \ldots \ldots \ldots, 7$

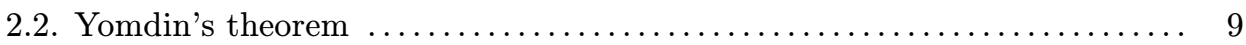

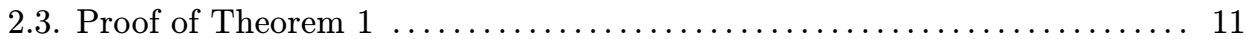

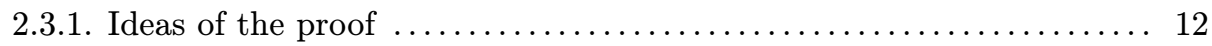

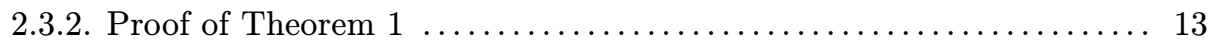

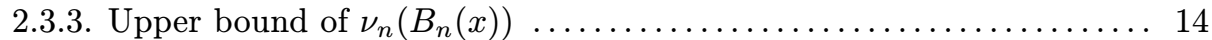

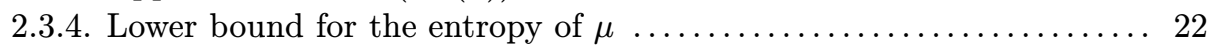

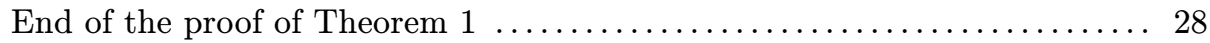

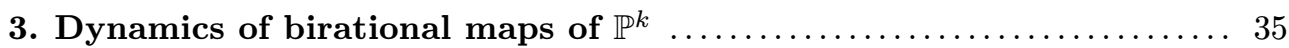

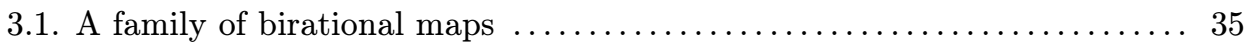

3.2. Construction of the Green currents $\ldots \ldots \ldots \ldots \ldots \ldots \ldots \ldots \ldots \ldots \ldots \ldots \ldots$

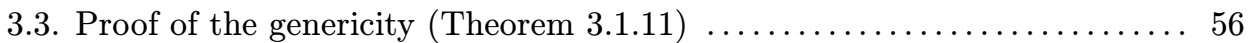

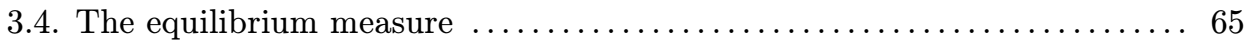

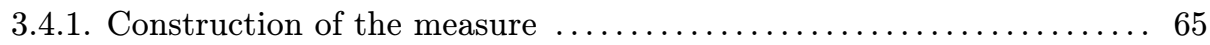

3.4.2. Green currents of order $1 \leq q \leq s \ldots \ldots \ldots \ldots \ldots \ldots \ldots \ldots \ldots \ldots$

3.4.3. Mixing, entropy and hyperbolicity of $\mu \ldots \ldots \ldots \ldots \ldots \ldots \ldots \ldots \ldots \ldots$

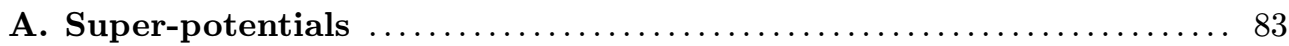

A.1. Definitions and properties of super-potentials $\ldots \ldots \ldots \ldots \ldots \ldots \ldots \ldots . \ldots 3$

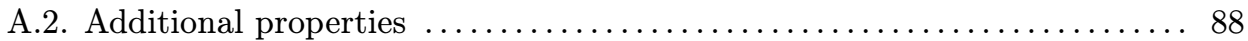

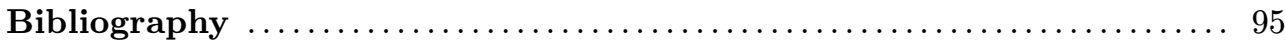





\section{CHAPTER 1}

\section{INTRODUCTION}

Let $X$ be a complex compact Kähler manifold of complex dimension $k$ endowed with a Kähler form $\omega$. We consider $f: X \rightarrow X$ a dominant meromorphic map and we denote by $I$ its indeterminacy set. We want to study the dynamics of $f$ in other words the behavior of the sequence of iterates $\left(f^{n}\right)$. We are particularly interested in the study of the ergodic properties of $f$.

Such study often starts with computing the topological entropy which gives an insight into how complicated the dynamics is. Classical objects in that case are ergodic measures of maximal entropy. Indeed, the support of such measure $\mu$ is an invariant set where the complexity of the dynamics is maximal. On this support, the dynamics is well understood in a statistical sense by Birkhoff's ergodic theorem as almost every orbit is equidistributed for the measure $\mu$. One can then study finer properties of the measure: mixing, speed of mixing, dimension of the measure ...

In order to understand more precisely the dynamics near a point $x$ in the support of the measure, one classicaly tries to compute the Lyapunov exponents. Roughly speaking, if no Lyapunov exponent is zero, these are numbers which give a rate of contraction and a rate of expansion in some stable and unstable manifolds. In other words, on the stable manifold the orbit of a point tends to the orbit of $x$ at a speed given by some negative Lyapunov exponent and on the unstable manifold the backward orbit of a point tends to the backward orbit of $x$ at a speed given by some positive Lyapunov exponent. When no Lyapunov exponent is zero, the measure is said to be hyperbolic. Finding examples of hyperbolic measures is a central question in dynamics and complex dynamics provides usually many of those (see [45] for definitions and results on hyperbolic measures).

In the particular case of complex dynamics, the topological entropy is related to the dynamical degrees. For $l=0 \ldots k$, we write:

$$
\lambda_{l}(f):=\int_{X} f^{*}\left(\omega^{l}\right) \wedge \omega^{k-l} .
$$


The l-th dynamical degree of $f$ is defined by (see [48] and [22]):

$$
d_{l}:=\lim _{n \rightarrow+\infty}\left(\lambda_{l}\left(f^{n}\right)\right)^{1 / n} .
$$

The degree $d_{l}$ measures the asymptotic spectral radius of the action of $f^{*}$ on the cohomology group $H^{l, l}(X)$. The last degree $d_{k}$ is the topological degree. It can be shown that the sequence of degrees is increasing up to a rank $s$ and then it is decreasing (see [40]). These quantities are of algebraic nature and there is a bound from above of the topological entropy by $\max _{0 \leq s \leq k} \log d_{s}$ (see [41] for the holomorphic case and $[\mathbf{2 5}],[\mathbf{2 2}]$ for the meromorphic case).

When one of the dynamical degree is strictly higher than the others, $f$ is called cohomologically hyperbolic. In that case, it is expected that there exists a measure of maximal entropy $\max _{0 \leq s \leq k} \log d_{s}$ (see [43]). Such measure is expected to be hyperbolic (and the saddle points are expected to be equidistributed along the measure). Recall the first author's result on Lyapunov exponents (Corollary 3 in [11]) when $f$ is cohomologically hyperbolic: if one can find a measure $\mu$ of entropy $\max _{0 \leq s \leq k} \log d_{s}$, then it is hyperbolic with estimates on the Lyapunov exponents provided that $\log \operatorname{dist}(x, I \cup C) \in L^{1}(\mu)$ ( $I$ is the indeterminacy set of $f$ and $C$ the critical set). In particular, in order to find hyperbolic measures, it is enough to find measures of maximal entropy.

This will be our aim in the first part of this article (Chapter 2). Following Yomdin's approach $([55])$, we give a criterion that allows us to produce invariant measures of maximal entropy for a meromorphic map on a compact Kähler manifold $X$. In a second part (Chapter 3), we study the more precise case of a family of birational maps of $\mathbb{P}^{k}$ for which we construct the equilibrium measure. We show that it is mixing and using the results of the first part we show that it is of maximal entropy. In particular the measure is hyperbolic. Let us detail our results.

Let $d$ denote the distance in $X$ and recall that $I$ is the indeterminacy set of $f$. In Chapter 2, we do not assume that $f$ is cohomologically hyperbolic. Chapter 2 is devoted to the proof of the following theorem:

THEOREM 1. - Consider the sequence of measures:

$$
\mu_{n}:=\frac{1}{n} \sum_{i=0}^{n-1} f_{*}^{i}\left(\frac{\left(f^{n}\right)^{*} \omega^{l} \wedge \omega^{k-l}}{\lambda_{l}\left(f^{n}\right)}\right) .
$$

Assume that there exists a converging subsequence $\mu_{\psi(n)} \rightarrow \mu$ with:

$$
(H): \lim _{n \rightarrow+\infty} \int \log d(x, I) d \mu_{\psi(n)}(x)=\int \log d(x, I) d \mu(x)>-\infty .
$$

Then $\mu$ is an invariant measure of metric entropy larger than or equal to $\log d_{l}$. 
In the above theorem, when $I=\varnothing$, we define $d(x, I):=1$ for all $x \in X$ so Hypothesis $(H)$ is automatically satisfied for holomorphic mappings.

Proving the convergence of the sequence $\mu_{n}$ with the hypothesis $(H)$ for $l=s$ such that $d_{s}$ is the highest dynamical degree gives measures of maximal entropy $\log d_{s}$. So, as we explained before, Theorem 1 is particularly interesting when $f$ is cohomologically hyperbolic and $l=s$ with $d_{s}$ the highest dynamical degree. In fact, in that case it is likely that $\left(\mu_{n}\right)$ will always converge to a measure of maximal entropy because this is the case in a lot of known cases (Henon maps [4] and [3], regular automorphisms of $\mathbb{C}^{k}[\mathbf{5 0}]$, endomorphisms of $\mathbb{P}^{k}[\mathbf{3 7}],[\mathbf{3 6}]$ and [38], some automorphisms on compact Kähler manifolds [7] and [24], ...).

We discuss Hypothesis $(H)$. First $\int \log d(x, I) d \mu(x)>-\infty$ is a natural hypothesis in order to produce hyperbolic measure because it is necessary to define the Lyapunov exponents $([\mathbf{1 1}])$. It would be a real improvement of Theorem 1 to prove the same result under that weaker hypothesis. Nevertheless, it is not always satisfied by meromorphic maps. Indeed, in [19] they are examples modifying Favre's examples (see [34]) for which $\log d(x, I)$ is not integrable with respect to the measure $\mu$. On the other hand, semi-continuity of the logarithm implies that

$$
\limsup _{n \rightarrow+\infty} \int \log d(x, I) d \mu_{\psi(n)}(x) \leq \int \log d(x, I) d \mu(x)
$$

so there really is only the other inequality to prove.

We explain the main ideas of the proof of Theorem 1. If $f$ is a Hénon map of $\mathbb{C}^{2}$, Bedford and Smillie have shown in [4] that the Green measure of $f$ is of maximal entropy. Their proof is based on Yomdin's theorem (see [55]) and also on the proof of the variational principle. This approach has been used several times since then in dynamics in order to bound from below the entropy of measures (e.g. [42], [10] and [32]). In all these cases, one can use Yomdin's theorem directly because the application $f$ is either holomorphic or when it is meromorphic everything takes place in a stable open set where $f$ is holomorphic.

The purpose of the first part is to quantify Bedford and Smillie's approach. We will need for that to modify the Bowen ball so that it takes into account the distance to the indeterminacy set $I$ and to quantify Yomdin's theorem. This is the main difficulty. We then use the proof of the variational principle to conclude.

Observe that the criterion can be extended to the case where $(X, \omega)$ is a compact Hermitian manifold. In that case, we do not know if the limit:

$$
d_{l}:=\lim _{n \rightarrow+\infty}\left(\lambda_{l}\left(f^{n}\right)\right)^{1 / n}
$$

exists, but it is sufficient to replace $d_{l}$ by $\lim \sup _{n}\left(\lambda_{l}\left(f^{\psi(n)}\right)\right)^{1 / \psi(n)}$ in the theorem.

In Chapter 3, we work in $\mathbb{P}^{k}$ endowed with the Fubini Study form $\omega$. We study the dynamics of some birational maps $f$ of $\mathbb{P}^{k}$, that is maps that are meromorphic and 
biholomorphic outside some analytic sets. Choose some $1 \leq s \leq k-1$. We assume that $\operatorname{dim}\left(I^{+}\right)=k-s-1$ and $\operatorname{dim}\left(I^{-}\right)=s-1$ where $I^{ \pm}$are the indeterminacy sets of $f^{ \pm}$(for $k=2$ the only possibility is $s=1$ and $I^{ \pm}$are points). Let $d$ and $\delta$ be the algebraic degrees of $f$ and $f^{-1}$.

Let $\mathcal{B}_{0}$ be the set of these birational maps of $\mathbb{P}^{k}$ such that:

$$
\bigcup_{n \geq 0} f^{-n} I^{+} \cap \bigcup_{n \geq 0} f^{n} I^{-}=\varnothing .
$$

For such a map, we have $\lambda_{s}\left(f^{n}\right)=d^{s n}$ and we can define $L^{n}\left(\omega^{s}\right):=d^{-s n}\left(f^{n}\right)^{*}\left(\omega^{s}\right)$ the sequence of normalized pull-backs and $\Lambda^{n}\left(\omega^{k-s}\right):=d^{-s n}\left(f^{-n}\right)^{*}\left(\omega^{k-s}\right)$ the sequence of normalized push-forwards. The key point of our method is the use of superpotentials introduced by Dinh and Sibony in [31]. We sum up the properties of the super-potentials that we need in an appendix. Super-potentials are a generalization of potentials to positive closed currents of bidegree higher than 1 . Let $\mathscr{C}_{l}$ be the set of positive closed current of bidegree $(l, l)$ and mass 1 . Then for $S \in \mathscr{C}_{l}$, the superpotential $\mathcal{U}_{S}$ of $S$ is a function on $\mathscr{C}_{k-l+1}$ that is uniquely determined by the condition $\mathcal{U}_{S}\left(\omega^{k-l+1}\right)=0$.

Let $\mathcal{U}_{L^{n}\left(\omega^{s}\right)}$ and $\mathcal{U}_{\Lambda^{n}\left(\omega^{k-s}\right)}$ be the super-potentials of $L^{n}\left(\omega^{s}\right)$ and $\Lambda^{n}\left(\omega^{k-s}\right)$. We study the set $\mathscr{B}$ of map $f \in \mathscr{B}_{0}$ such that:

$$
\lim _{n} U_{L^{n}\left(\omega^{s}\right)}\left(\left[I^{-}\right]_{\text {nor }}\right)>-\infty \quad \text { and } \quad \lim _{n} U_{\Lambda^{n}\left(\omega^{k-s}\right)}\left(\left[I^{+}\right]_{\text {nor }}\right)>-\infty,
$$

where $\left[I^{ \pm}\right]_{\text {nor }}:=\operatorname{vol}\left(I^{ \pm}\right)^{-1}\left[I^{ \pm}\right]$is the normalization of the current of integration on $I^{ \pm}$(we use in fact a slightly different definition that turns out to be equivalent to (1) by Corollary 3.2.5).

We prove in Theorem 3.2.8 below that (1) is implied by the more geometric condition:

$$
\begin{aligned}
& \sum_{n \geq 0}\left(\frac{1}{d}\right)^{n} \log \operatorname{dist}\left(I^{+}, f^{n}\left(I^{-}\right)\right)>-\infty \\
& \text { and } \\
& \sum_{n \geq 0}\left(\frac{1}{\delta}\right)^{n} \log \operatorname{dist}\left(I^{-}, f^{-n}\left(I^{+}\right)\right)>-\infty .
\end{aligned}
$$

This condition was in fact introduced by Bedford and Diller in [1] for the case of projective surface and is equivalent to (1) in this case. Using that hypothesis, the authors define the equilibrium measure and show that the potential of the Green current is integrable for the measure. They proved that the measure is mixing and hyperbolic. Using laminar currents, Dujardin computed the entropy and showed that the measure is of maximal entropy [33]. Diller and Guedj extended some parts of 
these results to a more general case in [19]. Note also the generalization of those ideas to the case of meromorphic maps of a surface in the recent articles [16], [17], [18].

When $k \geq 3$, the condition (1) is weaker than the geometric condition. This is due to the fact that distance between supports is a good distance for measure but not for current of higher bidimension. The interest of this condition is that it is generic in the following sense:

THEOREM 2. - Let $E_{s}$ be the set of birational maps $f: \mathbb{P}^{k} \rightarrow \mathbb{P}^{k}$ such that $I^{+}$and $I^{-}$satisfy $\operatorname{dim}\left(I^{+}\right)=k-s-1$ and $\operatorname{dim}\left(I^{-}\right)=s-1$. Consider the group action:

$$
\begin{aligned}
\Phi: \operatorname{PGL}(k+1, \mathbb{C}) \times E_{s} & \rightarrow E_{s} \\
(A, f) & \mapsto A \circ f .
\end{aligned}
$$

Then outside a pluripolar set of the orbit $\operatorname{Orb}(f)$ of $f \in E_{s}$, the maps of Orb $(f)$ are in $\mathcal{B}$.

Section 3.3 is devoted to the proof of that statement. It is interesting to note that the condition (1) is not generic in the set of birational maps (assuming one can give a sense to that statement). Indeed, the condition $\operatorname{dim}\left(I^{+}\right)=k-s-1$ and $\operatorname{dim}\left(I^{-}\right)=s-1$ is true for $k=2$ but does not need to be satisfied in higher dimension (see [44] and [42] for examples).

We sum up our results. We will construct the Green current of order $s$ and $k-s$ of $f$ and $f^{-1}$ for $f \in \mathcal{B}$. More precisely, we have (see Theorems 3.2.1, 3.2.9 and 3.2.19):

TheOREM 3. - Let $f \in \mathcal{B}$, then the sequence $L^{n}\left(\omega^{s}\right)$ is a well defined sequence of currents which converges in the sense of currents to a positive closed current $T_{s}^{+}$of bidegree $(s, s)$ and of mass 1 .

The current $T_{s}^{+}$satisfies $f^{*}\left(T_{s}^{+}\right)=d^{s} T_{s}^{+}$and is extremal in the set of positive closed currents.

Then we define the intersection $T_{s}^{+} \wedge T_{k-s}^{-}$and we prove (Theorem 3.4.1, Proposition 3.4.4 and Theorem 3.4.15):

THEOREM 4. - The wedge-product $\mu:=T_{s}^{+} \wedge T_{k-s}^{-}$is a well defined invariant probability measure for which the potential of the Green current of order 1 is integrable. The measure $\mu$ is mixing for $f$.

Using a space of test functions introduced by Dinh and Sibony in [26] and studied by the second author [53], we prove that the measure gives no mass to pluripolar sets. In particular, the measure gives no mass to analytic sets.

Then we use the results of Chapter 2 to prove that (Theorem 3.4.19, Theorem 3.4.21): 
Theorem 5. - The measure $\mu$ is of maximal entropy $\log d^{s}$ and is hyperbolic. More precisely, the Lyapunov exponents $\chi_{1} \geq \chi_{2} \geq \cdots \geq \chi_{k}$ of $\mu$ are well defined and we have the estimates:

$$
\begin{array}{r}
\chi_{1} \geq \cdots \geq \chi_{s} \geq \frac{1}{2} \log \frac{d_{s}}{d_{s-1}}=\frac{1}{2} \log d>0 \\
0>-\frac{1}{2} \log \delta=\frac{1}{2} \log \frac{d_{s+1}}{d_{s}} \geq \chi_{s+1} \geq \cdots \geq \chi_{k} .
\end{array}
$$

REMARK 1.0.1. - In these settings, it is natural to ask whether we have the equidistribution of the saddle points for $\mu$ ([43]). In the case of $\mathbb{P}^{2}$, the author of [1] prove the (weaker) result that the support of $\mu$ is contained in the closure of the saddle periodic points and Dujardin proved the equidistribution in [33]. We do not know how to prove such results in our case.

The main difficulty and novelty of that study is that in order to prove the convergences, we deal directly with positive closed currents of bidegree $(s, s)$. When $s>1$, the potentials $U$ of a positive closed current $S$ of bidegree $(s, s)$ are no longer quasiplurisubharmonic (qpsh for short) functions but currents satisfying $d d^{c} U+\omega^{s}=S$. Two such potentials $U$ and $U^{\prime}$ differ by a $d d^{c}$ closed current. Such object can be singular. So we use the new theory of super-potential ([31] and also [32] for the Kähler case). It provides a calculus on $(s, s)$ positive closed currents. We sum up the properties of super-potentials that we used in an appendix.

The two parts are fairly independent as we only use the results of Chapter 2 at the end of Chapter 3. So they can be read in any order.

Acknowledgements. - We thank the referee for his remarks and in particular for the simplification of the first point of the proof of proposition 2.3.8.

We also thank T.-C. Dinh for helping us constructing an example stable by perturbations in the proof of Theorem 3.1.11. 


\section{CHAPTER 2}

\section{ENTROPY OF MEROMORPHIC MAPS}

\subsection{Push-forward of measures by meromorphic maps}

Let $(X, \omega)$ be a compact Kähler manifold of dimension $k$. Modulo a normalization of the distance, we can assume that the diameter of $X$ is less than 1 . Let $f$ be a dominating meromorphic map and let $I$ be the indeterminacy set of $f$. Recall that for $l=0 \ldots k$, we write:

$$
\lambda_{l}(f):=\int_{X} f^{*}\left(\omega^{l}\right) \wedge \omega^{k-l} .
$$

We recall first how to define the push-forward by $f$ of a measure that gives no mass to $I$. In all that follows, a measure will mean a finite positive Radon measure.

Let $\nu$ be such a measure. On $X \backslash I, f$ is a measurable map. So we can define $f_{*} \nu$ by the formula:

$$
\left(f_{*} \nu\right)(A):=\nu(\{x \in X \backslash I \text { with } f(x) \in A\})=\nu\left(f^{-1}(A) \cap(X \backslash I)\right) .
$$

When a measure $\nu$ gives no mass to the indeterminacy set, we have:

$$
\int \varphi \circ f d \nu=\int \varphi d\left(f_{*} \nu\right)
$$

for all $\varphi \in L^{1}\left(f_{*} \nu\right)$. It is implicitly assumed that the integral is on $X \backslash I$. The equality follows from the approximation of function in $L^{1}$ by characteristic functions.

The operator $f_{*}$ has the good property of continuity. Indeed, we have:

LEMMA 2.1.1. - Let $\nu_{n}$ be a sequence of measures that give no mass to $I$. Then if $\left(\nu_{n}\right)$ converges to $\nu$ and $\nu(I)=0$ then $\left(f_{*}\left(\nu_{n}\right)\right)$ converges to $f_{*} \nu$.

Proof. - Let $\varphi$ be a continuous function and let $0 \leq \chi_{\varepsilon} \leq 1$ be a smooth function equal to 0 in an $\varepsilon$-neighborhood $I_{\varepsilon}$ of $I$ and equal to 1 outside a $2 \varepsilon$-neighborhood $I_{2 \varepsilon}$ of $I$. Then, we have:

$$
\int \varphi d\left(f_{*} \nu_{n}\right)=\int \varphi \circ f d \nu_{n}=\int\left(1-\chi_{\varepsilon}\right) \varphi \circ f d \nu_{n}+\int \chi_{\varepsilon} \varphi \circ f d \nu_{n} .
$$


The first term is bounded in absolute value by $\|\varphi\|_{\infty} \nu_{n}\left(I_{2 \varepsilon}\right)$ which can be taken arbitrarily small by taking $\varepsilon$ small then $n$ large (because $\nu$ gives no mass to $I$ ). The second term converges to $\int \chi_{\varepsilon} \varphi \circ f d \nu$ since $\chi_{\varepsilon} \varphi \circ f$ is a continuous function. Finally, if $\varepsilon$ is small enough, $\int \chi_{\varepsilon} \varphi \circ f d \nu$ is as close as we want from $\int \varphi \circ f d \nu$ since $\nu$ gives no mass to $I$.

In this section, we consider in particular the push-forward of the measures

$$
\nu_{n}:=\frac{\left(f^{n}\right)^{*} \omega^{l} \wedge \omega^{k-l}}{\lambda_{l}\left(f^{n}\right)} .
$$

The $\nu_{n}$ are well defined probability measures. Indeed, $\left(f^{n}\right)^{*} \omega^{l}$ is a form with coefficients in $L^{1}$ so it gives no mass to analytic sets of dimension $<k$. This implies that

$$
\frac{\left(f^{n}\right)^{*} \omega^{l} \wedge \omega^{k-l}}{\lambda_{l}\left(f^{n}\right)}
$$

is a probability that gives no mass to $\cup_{i \in \mathbb{N}} f^{-i}(I)$ (because $f$ is dominating). So we can push-forward this probability by $f^{i}$ and we get again a probability. We also make the observation:

$$
\left(f^{i}\right)_{*}\left(f^{j}\right)_{*} \frac{\left(f^{n}\right)^{*} \omega^{l} \wedge \omega^{k-l}}{\lambda_{l}\left(f^{n}\right)}=\left(f^{i+j}\right)_{*} \frac{\left(f^{n}\right)^{*} \omega^{l} \wedge \omega^{k-l}}{\lambda_{l}\left(f^{n}\right)},
$$

since $\left(f^{j}\right)^{-1}(I)$ is at most a hypersurface. In particular, we have $f_{*}^{i} \nu_{n}=\left(f^{i}\right)_{*} \nu_{n}$.

Now, we say that a measure $\mu$ that gives no mass to $I$ is invariant (or $f_{*}$-invariant) if $f_{*}(\mu)=\mu$. One has the following easy lemma:

LEMma 2.1.2. - Let $\mu$ be a measure that gives no mass to $I$. Then the following properties are equivalent:

- $\mu$ is invariant.

- For any continuous function $\varphi$, we have:

$$
\int \varphi \circ f d \mu=\int \varphi d \mu
$$

where the left-hand side integral is taken over $X \backslash I$.

When these properties are true, we even have:

$$
\int \varphi \circ f d \mu=\int \varphi d \mu
$$

for any $\varphi$ in $L^{1}(\mu)$ (with the same abuse of notation for the left-hand side integral that we will do in the whole section). 
Let us give some properties of meromorphic maps that will be useful in the proof of Theorem 1. First recall that we denote:

$$
\mu_{n}:=\frac{1}{n} \sum_{i=0}^{n-1} f_{*}^{i}\left(\frac{\left(f^{n}\right)^{*} \omega^{l} \wedge \omega^{k-l}}{\lambda_{l}\left(f^{n}\right)}\right) .
$$

We have seen that it is a well defined sequence of probabilities. Since $f$ is dominating, these measures give no mass to analytic sets of dimension $<k$ so we can define their push-forward.

We need an invariant measure to consider the metric entropy. So we will need the following lemma:

LEMMA 2.1.3. - If $\left(\mu_{\psi(n)}\right)$ converges to a measure $\mu$ that gives no mass to $I$, then $\mu$ is $f_{*}$-invariant.

Proof. - To simplify the notations, assume that $\left(\mu_{n}\right)$ converges to $\mu$.

We can write $f_{*}\left(\mu_{n}\right)=\mu_{n}+\alpha_{n}$ with $\alpha_{n}$ going to zero. Using Lemma 2.1.1, $f_{*}\left(\mu_{n}\right)$ converges to $f_{*} \mu$ and the lemma follows.

Now since we have an invariant probability measure that gives no mass to $I$, its mass is 1 on $\Omega=X \backslash \cup_{i \in \mathbb{N}} f^{-i}(I)$. Since $f(\Omega) \subset \Omega$, we can define the metric entropy of $\mu$ using partitions (see [43] and [45]).

We recall the following estimate that we use later:

LEMMA 2.1.4. - (see [20] Lemma 2.1)

There exist constants $K$ and $p$ such that:

$$
\|D f(x)\| \leq K d(x, I)^{-p} .
$$

\subsection{Yomdin's theorem}

In this paragraph, we recall some facts on Yomdin's theorem (see [55]) using Gromov's version (see [39] and [6]).

Let $l$ be an integer between 1 and $2 k$. If $Y$ is a subset of $\mathbb{C}^{k}$ (for example a submanifold of real dimension $l$ ), we call $C^{r}$-size (with $r \in \mathbb{N}^{*}$ ) of $Y$, the lower bound of the numbers $t \geq 0$ for which there exists a $C^{r}$-map of the unit l-cube into $\mathbb{C}^{k}$, $h:[0,1]^{l} \mapsto \mathbb{C}^{k}$, with $Y \subset h\left([0,1]^{l}\right)$ and $\left\|D_{r} h\right\| \leq t$. Here $D_{r} h$ is the vector assembled of (the components of) the partial derivatives of $h$ of order $1, \ldots, r$. The norm refers to supremum over $x \in[0,1]^{l}$ :

$$
\left\|D_{r} h\right\|=\sup _{x}\left\|D_{r} h(x)\right\| .
$$

We make some comments on $C^{r}$-size first. 
First, the $C^{1}$-size bounds the (real) $l$-dimensional volume of $Y$ and its diameter. More precisely

$$
C^{1}-\text { size of } Y \geq \max \left((\text { l-dimensional volume }(Y))^{1 / l}, l^{-1 / 2} \operatorname{Diameter}(Y)\right) .
$$

A process that we will use in what follows is the division of a set of $C^{r}$-size. If $Y$ is a set of $C^{r}$-size smaller than $t$, we can divide $Y$ in $j^{l}$ pieces of $C^{r}$-size smaller than $t / j$. For that it is sufficient to divide the $l$-cube $[0,1]^{l}$ in $j^{l}$ equal pieces and then to scale: for example $R:[0,1]^{l} \mapsto\left[0, j^{-1}\right]^{l}$ and similarly for the $j^{l}-1$ other cubes. The composition of $h:[0,1]^{l} \mapsto \mathbb{C}^{k}$ which covers $Y$ with the scaling $R$ satisfies $\left\|D_{r}(h \circ R)\right\| \leq t / j$ and the union of the images of these $j^{l}$ maps covers $Y$.

Here is now the principal result of Gromov-Yomdin that we will need (see Lemma 3.4 in [39]).

ThEOREM 2.2.1 ([39]). - Let $Y$ be an arbitrary subset in the graph $\Gamma_{g} \subset[0,1]^{l} \times \mathbb{C}^{k}$ of a $C^{r}$-map $g:[0,1]^{l} \mapsto \mathbb{C}^{k}$ and take some positive number $\epsilon \leq 1$. Then $Y$ can be divided into $N \leq C(k, l, r) \epsilon^{-l}\left(1+\left\|\partial_{r} g\right\|\right)^{l / r}$ sets of $C^{r}$-size $\leq C(k, l, r) \epsilon \operatorname{Diameter}(Y)$, where $\partial_{r} g$ denotes the vector assembled of the partial derivatives of $g$ of order exactly $r$ and $C(k, l, r)$ is a universal constant.

Here is the application of the above theorem that we will use: it is a small variation of Corollary 3.5 in [39].

Proposition 2.2.2. - Let $V$ be an open set of $\mathbb{C}^{k}$ and $f: V \rightarrow \mathbb{C}^{k}$ a map of class $C^{r}$. Let $Y_{0} \subset V$ be a set of $C^{r}$-size smaller than 1 such that $d\left(Y_{0}, \partial V\right) \geq \sqrt{l}$. Then the intersection of $f\left(Y_{0}\right)$ with a ball of $\mathbb{C}^{k}$ of radius $\beta$ can be divided into $N \leq$ $C(k, l, r)\left(1+\frac{\left\|D_{r} f\right\|}{\beta}\right)^{l / r}$ pieces of $C^{r}$-size less than $\beta$.

Proof. - We want to divide $f\left(Y_{0}\right) \cap B(a, \beta)$ into pieces of $C^{r}$-size $\leq \beta$. If $H(a, 1 / \beta)$ denotes the homothety of center $a$ and ratio $1 / \beta$ in $\mathbb{C}^{k}$, it is equivalent to divide

$$
H(a, 1 / \beta)\left(f\left(Y_{0}\right) \cap B(a, \beta)\right)=H(a, 1 / \beta)\left(f\left(Y_{0}\right)\right) \cap B(a, 1)
$$

into sets of $C^{r}$-size less than 1.

By hypothesis, there exists a map $h:[0,1]^{l} \rightarrow \mathbb{C}^{k}$ of class $C^{r}$ with $\left\|D_{r} h\right\| \leq 1$ and $Y_{0} \subset h\left([0,1]^{l}\right)$. Define $g:=H(a, 1 / \beta) \circ f \circ h$. By the chain rule, we have

$$
\left\|D_{r} g\right\| \leq \frac{C^{\prime}(k, l, r)}{\beta}\left\|D_{r} f\right\|
$$

We apply now the previous theorem to $Y$ the graph of $g$ intersected with $[0,1]^{l} \times$ $B(a, 1)$. So we have that we can cover $Y$ by a number:

$$
N \leq C(k, l, r)\left(1+\frac{C^{\prime}(k, l, r)}{\beta}\left\|D_{r} f\right\|\right)^{l / r} \leq C(k, l, r)\left(1+\frac{\left\|D_{r} f\right\|}{\beta}\right)^{l / r}
$$


sets of $C^{r}$-size $\leq 1$ (changing the constant $C(k, l, r)$ if necessary). Since the image of $Y$ by the projection $[0,1]^{l} \times \mathbb{C}^{k} \mapsto \mathbb{C}^{k}$ covers $H(a, 1 / \beta)\left(f\left(Y_{0}\right)\right) \cap B(a, 1)$, the proposition follows.

\subsection{Proof of Theorem 1}

Let $d$ denote the distance in $X$ normalized such that the diameter of $X$ is less than 1 and recall that $I$ is the indeterminacy set of $f$. In this paragraph, we prove the following theorem:

THEOREM. - Consider the sequence of measures:

$$
\mu_{n}:=\frac{1}{n} \sum_{i=0}^{n-1} f_{*}^{i}\left(\frac{\left(f^{n}\right)^{*} \omega^{l} \wedge \omega^{k-l}}{\lambda_{l}\left(f^{n}\right)}\right) .
$$

Assume that there exists a converging subsequence $\mu_{\psi(n)} \rightarrow \mu$ with:

$$
(H): \lim _{n \rightarrow+\infty} \int \log d(x, I) d \mu_{\psi(n)}(x)=\int \log d(x, I) d \mu(x)>-\infty .
$$

Then $\mu$ is an invariant measure of metric entropy larger than or equal to $\log d_{l}$.

In the above theorem, when $I=\varnothing$, we define $d(x, I):=1$ for all $x \in X$ so Hypothesis $(H)$ is automatically satisfied for holomorphic mappings.

Remark that the criterion can be extended to the case where $(X, \omega)$ is a compact Hermitian manifold. In that case, we do not know if the limit:

$$
d_{l}:=\lim _{n \rightarrow+\infty}\left(\lambda_{l}\left(f^{n}\right)\right)^{1 / n}
$$

exists, but it is sufficient to replace $d_{l}$ by $\lim \sup _{n}\left(\lambda_{l}\left(f^{\psi(n)}\right)\right)^{1 / \psi(n)}$ in the theorem.

We can also generalize the theorem in the following way: in the definition of $\mu_{n}$, we can put $\Omega_{1, l}$ instead of $\omega^{l}, \Omega_{2, k-l}$ instead of $\omega^{k-l}$ and $\Lambda_{l}\left(f^{n}\right)=\int\left(f^{n}\right)^{*} \Omega_{1, l} \wedge \Omega_{2, k-l}$ instead of $\lambda_{l}\left(f^{n}\right)$ with $\Omega_{1, l}$ a $(l, l)$ smooth form, $\Omega_{2, k-l}$ a $(k-l, k-l)$ smooth form such that one of them is weakly positive, the other is strongly positive and $\Lambda_{l}\left(f^{n}\right)>0$. Then, we have the same theorem by replacing $d_{l}$ by $\lim \sup _{n}\left(\Lambda_{l}\left(f^{\psi(n)}\right)\right)^{1 / \psi(n)}$.

In the previous theorem, it is possible to have that the metric entropy of $\mu$ is $>$ $\log d_{l}$. Indeed, if we take for $f$ a Lattès of $\mathbb{P}^{1}$ of degree $d \geq 2$ and $l=0$ in the theorem, we obtain on one hand that $\mu_{n}=\frac{1}{n} \sum_{i=0}^{n-1} f_{*}^{i} \omega$ converges to the equilibrium measure $\mu$ (by using the Birkhoff's theorem since $\mu$ is equivalent to $\omega$ ) which has metric entropy equal to $\log d$ and on the other hand we have that that $\log d_{0}=\log 1=0<\log d$.

We begin by giving the ideas of the proof of the theorem. 
2.3.1. Ideas of the proof. - Recall that we consider

$$
\nu_{n}=\frac{\left(f^{n}\right)^{*} \omega^{l} \wedge \omega^{k-l}}{\lambda_{l}\left(f^{n}\right)} \text { and so } \mu_{n}=\frac{1}{n} \sum_{i=0}^{n-1} f_{*}^{i}\left(\frac{\left(f^{n}\right)^{*} \omega^{l} \wedge \omega^{k-l}}{\lambda_{l}\left(f^{n}\right)}\right)=\frac{1}{n} \sum_{i=0}^{n-1} f_{*}^{i} \nu_{n} .
$$

For the statement of the ideas, assume to simplify that $\mu_{n}$ converges to a measure $\mu$.

First, consider the case where $f$ is holomorphic. As in the article of Bedford and Smillie (see [4]), the proof is based in one hand on Yomdin's theorem and in the other hand on the Misiurewicz's proof of the variational principle. We denote by $B_{n}(x, \epsilon)$ the dynamical ball (or Bowen ball):

$$
B_{n}(x, \epsilon)=\left\{y \in X, \max _{0 \leq i \leq n-1} d\left(f^{i}(x), f^{i}(y)\right) \leq \epsilon\right\} .
$$

For $\delta>0$, by using Yomdin's theorem (see [55]), we can prove that $\nu_{n}\left(B_{n}(x, \epsilon)\right) \leq$ $e^{n \delta} / \lambda_{l}\left(f^{n}\right)$ by taking $\epsilon$ small and then $n$ large enough. This gives an estimation from below for the metric entropy $H_{\nu_{n}}\left(\mathscr{P}_{-n}\right)$ where $\mathscr{P}_{-n}=\mathscr{D} \vee f^{-1} \mathscr{D} \vee \cdots \vee f^{-n+1} \mathscr{P}$ with $\mathscr{D}$ a finite partition of $X$ with atoms of diameter less than $\epsilon$. Now, by using Misiurewicz's proof of the variational principle, it implies that $\frac{1}{q} H_{\mu_{n}}\left(\mathscr{D}_{-q}\right)$ is bounded from below by something similar to $-2 \delta+\frac{1}{n} \log \lambda_{l}\left(f^{n}\right)$ for $n$ large enough. Now, since the partition $\mathscr{D}$ is finite and if $\mu$ puts no mass on its boundary, we obtain

$$
\frac{1}{q} H_{\mu}\left(\mathscr{P}_{-q}\right) \geq-2 \delta+\log d_{l}
$$

and by taking $q \rightarrow \infty$, we have $h_{\mu}(f) \geq \log d_{l}$, which is the inequality that we want.

Now, suppose that $f$ is meromorphic. We want to apply the same strategy but the problem is the indeterminacy set.

Namely, the previous estimate on $\nu_{n}\left(B_{n}(x, \epsilon)\right)$ uses Yomdin's theorem and only works for $C^{\infty}$ maps. The first idea to solve this problem is to use dynamical balls which avoid the indeterminacy set, i.e. of the type

$$
B_{n}(x)=\left\{y \in X, d\left(f^{i}(x), f^{i}(y)\right) \leq \rho\left(f^{i}(x)\right) \text { for } i \in[0, n-1]\right\}
$$

with $\rho(x)<<d(x, I)$. On these balls, the derivatives of $f$ may be large, but we have some estimates on them, and so, we will prove using Yomdin's approach, that $\nu_{n}\left(B_{n}(x)\right) \leq e^{n \delta} / \lambda_{l}\left(f^{n}\right)$ for $n$ large enough on a set of good points of mass almost 1 for $\nu_{n}$.

This inequality implies a lower bound on $H_{\nu_{n}}\left(\mathscr{D}_{-n}\right)$ but for partitions $\mathscr{P}$ such that the atom $\mathscr{P}(x)$ which contains $x$ has diameter smaller than $\rho(x)$. In particular these partitions are countable. Using Misiurewicz's method, we obtain more or less that $\frac{1}{q} H_{\mu_{n}}\left(\mathscr{P}_{-q}\right)$ is bounded by below by something similar to $-2 \delta+\frac{1}{n} \log \lambda_{l}\left(f^{n}\right)$ for $n$ 
large enough. To conclude, we have to let $n$ goes to $\infty$ : the problem is that $\mathscr{P}_{-q}$ is countable but by using the hypothesis

$$
(H): \lim _{n \rightarrow+\infty} \int \log d(x, I) d \mu_{n}(x)=\int \log d(x, I) d \mu(x)>-\infty,
$$

we can overcome this difficulty and we obtain the lower bound

$$
\frac{1}{q} H_{\mu}\left(\mathscr{P}_{-q}\right) \geq-2 \delta+\log d_{l}
$$

Hypothesis $(\mathrm{H})$ implies that the partition $\mathscr{P}$ has finite entropy, and so if we take the limit $q \rightarrow \infty$, we obtain $h_{\mu}(f) \geq \log d_{l}$, which is again the inequality that we want.

2.3.2. Proof of Theorem 1. - The hypothesis we made assure us that there exists a subsequence $\left(\mu_{\psi(n)}\right)$ which converges to a measure $\mu$ with:

$$
(H): \lim _{n \rightarrow+\infty} \int \log d(x, I) d \mu_{\psi(n)}(x)=\int \log d(x, I) d \mu(x)>-\infty .
$$

In order to clarify the exposition, we shall write $\psi(n)=n$. We start with the definition of the dynamical ball $B_{n}(x)$ that we will use.

If $s(x)$ is a function on $X$ with values in $\mathbb{R}^{+}$, we define (see [47]):

$$
B(x, s, n, f):=\left\{y, d\left(f^{i}(x), f^{i}(y)\right) \leq s\left(f^{i}(x)\right) \text { for } i \in[0, n-1]\right\} .
$$

We shall use these dynamical balls using for $s(x)$ the particular functions $\rho(x)$ and $\eta(x)$ where :

$$
\rho(x)=\left(\frac{d(x, I) \times \cdots \times d\left(f^{m-1}(x), I\right)}{K^{m}}\right)^{p}
$$

(here $K$ and $p$ are the numbers defined at the end of Section 2.1 and $m \in \mathbb{N}^{*}$ will be chosen later) and:

$$
\eta(x)=\left(\frac{d(x, I)}{K}\right)^{p} .
$$

When $f$ is holomorphic (i.e. $I=\varnothing$ ), take $d(x, I):=1$ and $p=2$ in these expressions.

If $n \in \mathbb{N}$ is fixed, by the Euclidean algorithm, we write $n=\phi(n) m+r(n)$ with $0 \leq r(n)<m$. In what follows, we will consider the following dynamical balls:

$$
B_{n}(x):=B\left(x, \rho, \phi(n), f^{m}\right) \cap f^{-\phi(n) m+m}\left(B\left(f^{\phi(n) m-m}(x), \eta, r(n)+m, f\right)\right) .
$$

Now, as explained in the paragraph 2.3.1, we will prove that $\nu_{n}\left(B_{n}(x)\right) \leq$ $e^{n \delta} / \lambda_{l}\left(f^{n}\right)$ for $n$ large enough on a set of good points of mass almost 1 for $\nu_{n}$. In particular, we will have a lot of dynamical balls. In a second paragraph, we will use this fact to bound from below the entropy of $\mu$. 
2.3.3. Upper bound of $\nu_{n}\left(B_{n}(x)\right)$. - We give some notations first. First of all we can put on $X$ a family of chart $\left(\tau_{x}\right)_{x \in X}$ such that $\tau_{x}(0)=x, \tau_{x}$ is defined on $B\left(0, \epsilon_{0}\right) \subset \mathbb{C}^{k}$ with $\epsilon_{0}>0$ independent of $x$ and such that the norm of the derivatives of order 1 of the $\tau_{x}$ is bounded from above by a constant independent of $x$. These charts are obtained from a finite family $\left(U_{i}, \psi_{i}\right)$ of charts of $X$ by composing them with translations. In $\mathbb{C}^{k}$, we also consider $\pi_{1}, \ldots, \pi_{i}$ the projections from $\mathbb{C}^{k}$ onto the vectorial subspaces of dimension $k-l$. In what follows, the choice of these coordinates is supposed to be generic and $\beta_{j}$ denotes the standard volume form on $\pi_{j}\left(\mathbb{C}^{k}\right)$.

Fix $x \in X$ and:

$$
\Omega:=\left(\tau_{x}\right)_{*}\left(\pi_{1}^{*} \beta_{1}+\cdots+\pi_{i}^{*} \beta_{i}\right)
$$

We want to compute :

$$
\nu_{n}\left(B_{n}(x)\right)=\frac{\left(f^{n}\right)^{*} \omega^{l} \wedge \omega^{k-l}}{\lambda_{l}\left(f^{n}\right)}\left(B_{n}(x)\right) .
$$

Taking $K$ large enough, we can assume that $B_{n}(x) \subset \tau_{x}\left(B\left(0, \epsilon_{0}\right)\right)$ so the previous quantity is less than:

$$
C(X) \frac{\left(f^{n}\right)^{*} \omega^{l} \wedge \Omega}{\lambda_{l}\left(f^{n}\right)}\left(B_{n}(x)\right)=C(X) \sum_{j=1}^{i} \iint_{B_{n}(x) \cap \tau_{x}\left(Y_{j}(t)\right)} \frac{\left(f^{n}\right)^{*} \omega^{l}}{\lambda_{l}\left(f^{n}\right)} d t
$$

where $Y_{j}(t)$ is equal to $\pi_{j}^{-1}(t)$ for $t$ in the $j$-th subspace of dimension $k-l$ and $d t$ stands for the Lebesgue measure on that space (we used Fubini theorem: see [8] p. 334). Remark that $t$ lives in a ball $B\left(0, \epsilon_{0}\right)$.

So we have a upper bound of $\nu_{n}\left(B_{n}(x)\right)$ by:

$$
\frac{C(X)}{\lambda_{l}\left(f^{n}\right)} \sum_{j=1}^{i} \iint_{f^{n}\left(B_{n}(x) \cap \tau_{x}\left(Y_{j}(t)\right)\right)} \omega^{l} d t .
$$

To control this integral, we have to bound from above the $2 l$-dimensional volume of $f^{n}\left(B_{n}(x) \cap \tau_{x}\left(Y_{j}(t)\right)\right)$ for some good points $x$ of $\nu_{n}$. In order to do that, we explain first what are the good points for $\nu_{n}$ then we will bound the volume using Yomdin's approach and finally we will finish the bound of $\nu_{n}\left(B_{n}(x)\right)$.

Good points for the measure $\nu_{n}$. - In what follows, we consider a constant $L>0$ and an integer $n_{0}$ such that:

$$
\int \log d(x, I) d \mu_{n}(x) \geq-L,
$$

for $n \geq n_{0}$. The existence of these constants follows easily from Hypothesis $(\mathrm{H})$.

Let $\delta>0$. Our goal is to show that the entropy of $\mu$ is greater than $\log d_{l}-\delta$. We choose a constant $C_{0}$ large enough $\left(1 / C_{0} \ll \delta\right)$. 
We are going to show that Hypothesis $(\mathrm{H})$ implies that the orbits of generic points of the measure $\nu_{n}=\frac{\left(f^{n}\right)^{*} \omega^{l} \wedge \omega^{k-l}}{\lambda_{l}\left(f^{n}\right)}$ are not close to the indeterminacy set $I$. They are going to be the good points.

Lemma 2.3.1. - For $n \geq n_{0}$, there exists a set $A_{n}$ of $\nu_{n}$-measure greater or equal to $1-C_{0}^{-1}$ whose points $x \in A_{n}$ satisfy:

$$
\prod_{i \in[0, n-1]} d\left(f^{i}(x), I\right) \geq e^{-C_{0} L n} .
$$

Proof. - We have

$$
\frac{1}{n} \int \log \left(\prod_{i \in[0, n-1]} d\left(f^{i}(x), I\right)\right) d \nu_{n}(x)=\frac{1}{n} \int \sum_{i=0}^{n-1} \log d\left(f^{i}(x), I\right) d \nu_{n}(x) .
$$

Since $\mu_{n}=\frac{1}{n} \sum_{i=0}^{n-1}\left(f^{i}\right)_{*} \nu_{n}$ :

$$
\frac{1}{n} \int \log \left(\prod_{i \in[0, n-1]} d\left(f^{i}(x), I\right)\right) d \nu_{n}(x)=\int \log d(x, I) d \mu_{n}(x) .
$$

Thanks to our hypothesis, this last integral is $\geq-L$.

Now, if we denote $h(x)=\frac{1}{n} \log \left(\prod_{i \in[0, n-1]} d\left(f^{i}(x), I\right)\right)$ and $A_{n}:=\{x, h(x) \geq$ $\left.-C_{0} L\right\}$, we have:

$$
\int_{A_{n}} h(x) d \nu_{n}(x)+\int_{X \backslash A_{n}} h(x) d \nu_{n}(x) \geq-L .
$$

But $\int_{A_{n}} h(x) d \nu_{n}(x) \leq 0$ and $\int_{X \backslash A_{n}} h(x) d \nu_{n}(x) \leq-C_{0} L \nu_{n}\left(X \backslash A_{n}\right)$.

This implies that $\nu_{n}\left(X \backslash A_{n}\right) \leq 1 / C_{0}$.

The set $A_{n}$ is indeed of measure $\geq 1-C_{0}^{-1}$ and if $x \in A_{n}$ then:

$$
\prod_{i \in[0, n-1]} d\left(f^{i}(x), I\right) \geq e^{-C_{0} L n}
$$

which is what we wanted.

The orbit of points in $A_{n}$ are not too close to $I$. These are the good points for the measure $\nu_{n}$.

We now prove the upper bound of the volume. 
Upper bound for the volume of $f^{n}\left(B_{n}(x) \cap \tau_{x}\left(Y_{j}(t)\right)\right)$ for $x \in A_{n}$. - Let $Y_{0}$ denote one of the $\tau_{x}\left(Y_{j}(t)\right)$ (where $Y_{j}(t)$ is the fiber of $\pi_{j}$ with $t$ in the $j$-th subspace of dimension $k-l)$. Our aim is to prove:

Proposition 2.3.2. - The $2 l$-dimensional volume of $f^{n}\left(Y_{0} \cap B_{n}(x)\right)$ is less or equal to:

$$
C(X, l, r)^{n / m+2 m} \times K^{\frac{2 n p l}{r}+\frac{4 m p l}{r}} \times \prod_{0 \leq i \leq n-1} d\left(f^{i}(x), I\right)^{\frac{-4 p l}{r}} .
$$

Here $C(X, l, r)$ is a constant that depends only on $X$, of the complex dimension $l$ of $Y_{0}$ and the regularity $r$ that we chose. The constants $K=K(f)$ and $p=p(f)$ are those of paragraph 2.1 .

Observe that the upper bound does not depend on the fiber $Y_{j}(t)$ that we consider.

Before proving the proposition, we give the upper bound of the $2 l$-dimensional volume of $f^{n}\left(B_{n}(x) \cap \tau_{x}\left(Y_{j}(t)\right)\right)$ that follows from the proposition.

Recall that we fixed $\delta$ and $C_{0}$. Now, let $r$ be such that $\frac{1}{r} \log K<\delta$ and $\frac{C_{0} L}{r}<\delta$. Then, we choose $m$ so that $\frac{1}{m} \log (C(X, l, r))<\delta$ where $C(X, l, r)$ is the constant from the previous proposition. Reformulating the previous proposition we have that the $2 l$-dimensional volume of $f^{n}\left(Y_{0} \cap B_{n}(x)\right)$ is bounded by:

$$
C(X, l, r, m, p, K) e^{\delta n} \times e^{2 \delta n p l} \times \prod_{0 \leq i \leq n-1} d\left(f^{i}(x), I\right)^{\frac{-4 p l}{r}} .
$$

Finally, if $x$ is in $A_{n}$ (i.e. if $x$ is a good point for the measure $\nu_{n}$ ), the $2 l$-dimensional volume of $f^{n}\left(Y_{0} \cap B_{n}(x)\right)$ is bounded from above by (see Lemma 2.3.1):

$$
e^{4 \delta n p l} e^{\frac{4 p l C_{0} L n}{r}} \leq e^{8 \delta n p l},
$$

if $n$ is large (independently of $x \in A_{n}$ ).

It is this upper bound that we use now to finish the upper bound of $\nu_{n}\left(B_{n}(x)\right)$ for $x \in A_{n}$.

End of the proof of the upper bound of $\nu_{n}\left(B_{n}(x)\right)$ for $x \in A_{n}$

Recall that we have bounded $\nu_{n}\left(B_{n}(x)\right)$ by:

$$
\frac{C(X)}{\lambda_{l}\left(f^{n}\right)} \sum_{j=1}^{i} \iint_{f^{n}\left(B_{n}(x) \cap \tau_{x}\left(Y_{j}(t)\right)\right)} \omega^{l} d t .
$$

Now, if $x \in A_{n}$, we get:

$$
\nu_{n}\left(B_{n}(x)\right) \leq \frac{e^{10 \delta n p l}}{\lambda_{l}\left(f^{n}\right)},
$$

for $n$ large enough which does not depend on $x \in A_{n}$. This quantity is approximately $d_{l}^{-n}$ and it stands for $x \in A_{n}$ which is a set of measure $\geq 1-\frac{1}{C_{0}}$ for $\nu_{n}$. This is the upper bound that we wanted and it will allow us to bound the entropy of $\mu$. 
It remains to prove Proposition 2.3.2, which is the purpose of rest of this section.

Proof of Proposition 2.3.2. - Consider $g=f^{a}$ an iterate of $f$ and let $x \in X$. We define $g_{x}=\tau_{g(x)}^{-1} \circ g \circ \tau_{x}$. We also define $g_{x, s(x)}=h\left(0, \frac{1}{s(x)}\right) \circ g_{x} \circ h(0, s(x))$ where $h(0, t)$ is the homothety of center 0 and ratio $t$ in $\mathbb{C}^{k}$. Here, $s(x)$ is defined by:

$$
s(x)=s_{a}(x)=\left(\frac{d(x, I) \times \cdots \times d\left(f^{a-1}(x), I\right)}{K^{a}}\right)^{p} .
$$

We will consider later the particular cases $a=1$ (i.e. $s(x)=\eta(x)$ ) and $a=m$ (i.e. $s(x)=\rho(x))$.

In what follows, we are going to consider $C^{r}$-sizes associated to $2 l$ (i.e. sets that will be cover by some $h\left([0,1]^{2 l}\right)$ with $\left.h \in C^{r}\right)$. First, we prove the following lemma by induction:

Lemma 2.3.3. - Let $Z_{0}$ be a set of complex dimension $l$ such that the $C^{r}$-size of $\tau_{x}^{-1}\left(Z_{0} \cap B(x, s(x))\right)$ is $\leq s(x)$. Let $B(x, s, j, g)$ be the dynamical ball:

$$
B(x, s, j, g)=\left\{y, d\left(g^{i}(x), g^{i}(y)\right) \leq s\left(g^{i}(x)\right) \text { for } i \in[0, j-1]\right\} .
$$

Then, for $j \geq 1$, we can cover $g^{j-1}\left(Z_{0} \cap B(x, s, j, g)\right)$ by a union of $N_{j}$ sets $Z$ for which the $C^{r}$-size of $\tau_{g^{j-1}(x)}^{-1}(Z)$ is $\leq s\left(g^{j-1}(x)\right)$ and $N_{j}$ is bounded from above by:

$$
C(X, l, r)^{j-1} \prod_{0 \leq i \leq j-1} s\left(g^{i}(x)\right)^{-2 l / r} .
$$

Proof. - For $j=1$, the lemma stands by hypothesis.

Assume now that the induction assumption stands for $j-1$.

Observe that:

$$
g^{j-1}\left(Z_{0} \cap B(x, s, j, g)\right)=g\left(g^{j-2}\left(Z_{0} \cap B(x, s, j-1, g)\right)\right) \cap B\left(g^{j-1}(x), s\left(g^{j-1}(x)\right)\right) .
$$

This is true since $B(x, s, j, g)=B(x, s, j-1, g) \cap g^{-j+1} B\left(g^{j-1}(x), s\left(g^{j-1}(x)\right)\right)$ and $\varphi\left(A \cap \varphi^{-1}(B)\right)=\varphi(A) \cap B$ for any map $\varphi$ and any sets $A$ and $B$.

Let $Z$ be one of the $N_{j-1}$ sets whose union covers $g^{j-2}\left(Z_{0} \cap B(x, s, j-1, g)\right)$. The $C^{r}-$ size of $\tau_{g^{j-2}(x)}^{-1}(Z)$ is $\leq s\left(g^{j-2}(x)\right)$ by the induction assumption. To prove the lemma, we bound from above the numbers of sets $Y$ which cover $g(Z) \cap B\left(g^{j-1}(x), s\left(g^{j-1}(x)\right)\right)$ for which the $C^{r}$-size of $\tau_{g^{j-1}(x)}^{-1}(Y)$ is $\leq s\left(g^{j-1}(x)\right)$.

We consider $\widetilde{Z}=h\left(0,1 / s\left(g^{j-2}(x)\right)\right) \circ \tau_{g^{j-2}(x)}^{-1}(Z)$. The $C^{r}$-size of $\widetilde{Z}$ is $\leq s\left(g^{j-2}(x)\right) \times$ $\frac{1}{s\left(g^{j-2}(x)\right)}=1$. Furthermore, since $Z$ is in the ball $B\left(g^{j-2}(x), s\left(g^{j-2}(x)\right)\right)$ (else we only consider the part of $Z$ that is in the ball and we still denote it $Z$ ), $\widetilde{Z}$ is in the ball $B(0, C(X)$ ) (where $C(X)$ is a constant that depends only on $X$ ). Using Proposition 
2.2.2 of Section 2.2 with $f=g_{g^{j-2}(x), s\left(g^{j-2}(x)\right)}$ and $Y_{0}=\widetilde{Z}$ we get that we can cover $g_{g^{j-2}(x), s\left(g^{j-2}(x)\right)}(\widetilde{Z}) \cap B(0, \beta)$ (we take $\left.\beta=C(X) \frac{s\left(g^{j-1}(x)\right)}{s\left(g^{j-2}(x)\right)}\right)$ by

$$
C(X, l, r)\left(1+\frac{\left\|D_{r} g_{g^{j-2}(x), s\left(g^{j-2}(x)\right)}\right\|}{\beta}\right)^{2 l / r}
$$

sets $\tilde{Y}$ of $C^{r}$-size $\leq C(X) \frac{s\left(g^{j-1}(x)\right)}{s\left(g^{j-2}(x)\right)}$. Here the norm $\|\cdot\|$ is taken over the ball $B(0, C(X)+\sqrt{2 l})$. The images $Y$ of the $\tilde{Y}$ by $\tau_{g^{j-1}(x)} \circ h\left(0, s\left(g^{j-2}(x)\right)\right)$ cover

$$
\begin{aligned}
& \tau_{g^{j-1}(x)} \circ h\left(0, s\left(g^{j-2}(x)\right)\right)\left(g_{g^{j-2}(x), s\left(g^{j-2}(x)\right)}(\widetilde{Z}) \cap B(0, \beta)\right) \\
& =g(Z) \cap \tau_{g^{j-1}(x)} \circ h\left(0, s\left(g^{j-2}(x)\right)\right)(B(0, \beta))
\end{aligned}
$$

which contains

$$
g(Z) \cap B\left(g^{j-1}(x), s\left(g^{j-1}(x)\right)\right) .
$$

This is the set we wanted to cover and $\tau_{g^{j-1}(x)}^{-1}(Y)=h\left(0, s\left(g^{j-2}(x)\right)\right)(\tilde{Y})$ is of $C^{r}$-size $\leq s\left(g^{j-1}(x)\right)$ up to dividing it into $C(X)^{2 l}$ pieces as in Section 2.2 (this multiplies $N_{j}$ by a universal constant).

To finish the proof, we have to count the number of pieces $Y$ that we constructed for which the $C^{r}$-size of $\tau_{g^{j-1}(x)}^{-1}(Y)$ is bounded from above by $s\left(g^{j-1}(x)\right)$. Indeed, the union of those sets covers $g^{j-1}\left(Z_{0} \cap B(x, s, j, g)\right)$.

To control $N_{j}$, we need a control of the norm $\left\|D_{r} g_{g^{j-2}(x), s\left(g^{j-2}(x)\right)}\right\|$ on the ball $B(0, C(X)+\sqrt{2 l})$.

We admit temporarily that this norm is $\leq C(X, l, r) s\left(g^{j-2}(x)\right)^{-1}$.

Then:

$$
N_{j} \leq N_{j-1} C(X, l, r)\left(1+\frac{\left\|D_{r} g_{g^{j-2}(x), s\left(g^{j-2}(x)\right)}\right\| s\left(g^{j-2}(x)\right)}{s\left(g^{j-1}(x)\right)}\right)^{2 l / r},
$$

which is smaller than:

$$
N_{j-1} C(X, l, r)\left(\frac{2 C(X, l, r)}{s\left(g^{j-1}(x)\right)}\right)^{2 l / r} \leq N_{j-1} C(X, l, r) s\left(g^{j-1}(x)\right)^{-2 l / r}
$$

up to changing $C(X, l, r)$. This concludes the proof of the lemma up to the upper bound of the norm of $\left\|D_{r} g_{g^{j-2}(x), s\left(g^{j-2}(x)\right)}\right\|$ on the ball $B(0, C(X)+\sqrt{2 l})$.

Upper bound of the norm $\left\|D_{r} g_{g^{j-2}(x), s\left(g^{j-2}(x)\right)}\right\|$ on $B(0, C(X)+\sqrt{2 l})$. - Since

$$
g_{g^{j-2}(x), s\left(g^{j-2}(x)\right)}=h\left(0, \frac{1}{s\left(g^{j-2}(x)\right)}\right) \circ g_{g^{j-2}(x)} \circ h\left(0, s\left(g^{j-2}(x)\right)\right),
$$

$\left\|\partial_{r} g_{g^{j-2}(x), s\left(g^{j-2}(x)\right)}\right\|$ is equal to $s\left(g^{j-2}(x)\right)^{r-1}\left\|\partial_{r} g_{g^{j-2}(x)}\right\|$ where that last norm is taken over the ball

$$
B\left(0, s\left(g^{j-2}(x)\right)(C(X)+\sqrt{2 l})\right)
$$

(see Section 2.2 for notations). 
To prove the upper bound of the norm, we are going to prove that:

$$
g_{g^{j-2}(x)}\left(B\left(0,2 s\left(g^{j-2}(x)\right)(C(X)+\sqrt{2 l})\right)\right)
$$

is contained in the ball $B(0,1)$. We will then deduce the upper bound of $\left\|\partial_{r} g_{g^{j-2}(x)}\right\|$ on $B\left(0, s\left(g^{j-2}(x)\right)(C(X)+\sqrt{2 l})\right)$ by $C(X, r)\left(s\left(g^{j-2}(x)\right)(C(X)+\sqrt{2 l})\right)^{-r}$ thanks to Cauchy inequalities. This gives exactly the upper bound that we want.

If we let $y=g^{j-2}(x)$, we have:

$$
g_{g^{j-2}(x)}\left(B\left(0,2 s\left(g^{j-2}(x)\right)(C(X)+\sqrt{2 l})\right)\right)=g_{y}(B(0,2 s(y)(C(X)+\sqrt{2 l})))
$$

which is equal to:

$$
\tau_{f^{a}(y)}^{-1} \circ f^{a} \circ \tau_{y}(B(0,2 s(y)(C(X)+\sqrt{2 l})))
$$

because $g=f^{a}$. Furthermore:

$$
\tau_{f^{a}(y)}^{-1} \circ f^{a} \circ \tau_{y}=f_{f^{a-1}(y)} \circ \cdots \circ f_{y},
$$

with $f_{x}:=\tau_{f(x)}^{-1} \circ f \circ \tau_{x}$.

Now we use Lemma 2.1.4 of Section 2.1 to control the differential of $f_{y}$ on $B(0,2 s(y)(C(X)+\sqrt{2 l}))$.

If $z$ is a point of the ball $B(0,2 s(y)(C(X)+\sqrt{2 l}))$ then the distance between $\tau_{y}(z)$ and $I$ is $\geq d(y, I)-2 s(y) C(X)(C(X)+\sqrt{2 l})$. But that last quantity is $\geq \frac{d(y, I)}{2}$ since by definition of $s(y)$, we have $s(y) \leq \frac{d(y, I)}{K}$ and we can assume that $K$ is large compared to the constants that depend only on $X$ and $l$ (recall that $l$ is the complex dimension of $Z_{0}$ : it is between 0 and $k$, so in particular they are only a finite number of such quantities). Using Lemma 2.1.4, we get an upper bound of $\left\|D f_{y}\right\|$ on the ball $B(0,2 s(y)(C(X)+\sqrt{2 l}))$ by $K C(X) 2^{p} d(y, I)^{-p}$. Using the control over the differential, this implies that the image of $B(0,2 s(y)(C(X)+\sqrt{2 l}))$ by $f_{y}$ is contained in $B\left(0, K C(X) 2^{p} d(y, I)^{-p} 2 s(y)(C(X)+\sqrt{2 l})\right)$. But since:

$$
s(y)=\left(\frac{d(y, I) \times \cdots \times d\left(f^{a-1}(y), I\right)}{K^{a}}\right)^{p},
$$

we have:

$$
K C(X) 2^{p} d(y, I)^{-p} 2 s(y)(C(X)+\sqrt{2 l}) \leq\left(\frac{d(f(y), I) \times \cdots \times d\left(f^{a-1}(y), I\right)}{K^{a-1}}\right)^{p},
$$

since we can assume that $K$ is large compared to the $C(X)$.

So we have proved that the image of $B(0,2 s(y)(C(X)+\sqrt{2 l}))$ by $f_{y}$ is contained in

$$
B\left(0,\left(\frac{d(f(y), I) \times \cdots \times d\left(f^{a-1}(y), I\right)}{K^{a-1}}\right)^{p}\right)
$$


Now, if we do again what we just did for $f(y)$ instead of $y$, we get that the image by $f_{f(y)} \circ f_{y}$ of the ball $B(0,2 s(y)(C(X)+\sqrt{2 l}))$ is contained in the ball:

$$
B\left(0,\left(\frac{d\left(f^{2}(y), I\right) \times \cdots \times d\left(f^{a-1}(y), I\right)}{K^{a-2}}\right)^{p}\right),
$$

and so on. At the end, we have that the image of the ball $B(0,2 s(y)(C(X)+\sqrt{2 l}))$ by $f_{f^{a-1}(y)} \circ \cdots \circ f_{y}=\tau_{f^{a}(y)}^{-1} \circ f^{a} \circ \tau_{y}$ is contained in the ball:

$$
B\left(0, K C(X) 2^{p} d\left(f^{a-1}(y), I\right)^{-p}\left(\frac{d\left(f^{a-1}(y), I\right)}{K}\right)^{p}\right),
$$

which is contained in $B(0,1)$ for $K$ large enough (observe that such $K$ does not depend on $a$ as the only requirement is $K^{-p+1} C(X) 2^{p} \leq 1$ where $p \geq 2$ ).

This concludes the proof of the upper bound of the norm $\left\|D_{r} g_{g^{j-2}(x), s\left(g^{j-2}(x)\right)}\right\|$ on the ball $B(0, C(X)+\sqrt{2 l})$ and that concludes the proof of the lemma.

Now we will use that lemma to prove Proposition 2.3.2. Recall some notations first. The set $Y_{0}$ is one the fiber $\tau_{x}\left(Y_{j}(t)\right), n=m \phi(n)+r(n)$ with $0 \leq r(n)<m$,

$$
\rho(x)=\left(\frac{d(x, I) \times \cdots \times d\left(f^{m-1}(x), I\right)}{K^{m}}\right)^{p}
$$

and

$$
\eta(x)=\left(\frac{d(x, I)}{K}\right)^{p}
$$

Recall that:

$$
B_{n}(x)=B\left(x, \rho, \phi(n), f^{m}\right) \cap f^{-\phi(n) m+m}\left(B\left(f^{\phi(n) m-m}(x), \eta, r(n)+m, f\right)\right) .
$$

Applying the previous lemma for $g=f^{m}$ (and thus $\left.s(x)=\rho(x)\right), j=\phi(n)$ and $Z_{0}=$ $Y_{0} \cap B(x, \rho(x))$ (whose image by $\tau_{x}^{-1}$ is of $C^{r}$-size $\leq \rho(x)$ up to dividing into $C(X)^{2 l}$ pieces because $Y_{j}(t)$ is a linear subspace), we get that we can cover $f^{m(\phi(n)-1)}\left(Y_{0} \cap\right.$ $B(x, \rho, \phi(n), g))$ by a number $N_{\phi(n)}$ of sets $Z$ for which the $C^{r}$-size of $\tau_{g^{\phi(n)-1}(x)}^{-1}(Z)=$ $\tau_{f^{m(\phi(n)-1)}(x)}^{-1}(Z)$ is $\leq \rho\left(g^{\phi(n)-1}(x)\right)$ and $N_{\phi(n)}$ bounded from above by:

$$
C(X, l, r)^{\phi(n)} \prod_{0 \leq i \leq \phi(n)-1} \rho\left(g^{i}(x)\right)^{-2 l / r} .
$$

So we went up to $f^{m(\phi(n)-1)}(x)$ and we still have to go to $f^{n}(x)$.

For that, we use the above lemma again with for $Z_{0}$ one of the $N_{\phi(n)}$ pieces $Z, g=f($ so now $s(x)=\eta(x)), j=n-m(\phi(n)-1)=r(n)+m$ and $x=f^{m(\phi(n)-1)}(x)$. We can do that because the $C^{r}$-size of $\tau_{f^{m(\phi(n)-1)}(x)}^{-1}\left(Z_{0}\right)$ is $\leq \rho\left(f^{m(\phi(n)-1)}(x)\right) \leq \eta\left(f^{m(\phi(n)-1)}(x)\right)$. So we get that we can cover $f^{r(n)+m-1}(Z \cap$ 
$\left.B\left(f^{m(\phi(n)-1)}(x), \eta, r(n)+m, f\right)\right)$ by a union of $M$ sets $Y$ for which the $C^{r}$-size of $\tau_{f^{n-1}(x)}^{-1}(Y)$ is $\leq \eta\left(f^{n-1}(x)\right)$ and $M$ is less than:

$$
C(X, l, r)^{m+r(n)-1} \prod_{1 \leq i \leq m+r(n)} \eta\left(f^{n-i}(x)\right)^{-2 l / r} .
$$

The sets $Y$ that we constructed belong to (up to keeping the part that lies in it):

$$
B\left(f^{r(n)+m-1+m(\phi(n)-1)}(x), \eta\left(f^{r(n)+m-1+m(\phi(n)-1)}(x)\right)\right)=B\left(f^{n-1}(x), \eta\left(f^{n-1}(x)\right)\right) .
$$

The $C^{1}$-size of these $Y$ is smaller than $C(X) \eta\left(f^{n-1}(x)\right)$ which implies that the diameter of $h\left([0,1]^{2 l}\right.$ ) (where $h$ is the map in $C^{r}$ associated to $Y$ ) is smaller than $C(X, l) \eta\left(f^{n-1}(x)\right)$. So, the set $h\left([0,1]^{2 l}\right)$ is contained in

$$
B\left(f^{n-1}(x), \frac{d\left(f^{n-1}(x), I\right)}{2}\right) .
$$

Since the differential of $f$ in this last ball is bounded by

$$
K 2^{p} d\left(f^{n-1}(x), I\right)^{-p}
$$

using Lemma 2.1.4, one gets that the images by $f$ of those $Y$ are of $C^{1}$-size bounded by $C(X) \eta\left(f^{n-1}(x)\right) K 2^{p} d\left(f^{n-1}(x), I\right)^{-p}$. So their $2 l$-dimensional volume is $\leq 1$.

Summing up, we have covered

$$
f^{r(n)+m}\left(f^{m(\phi(n)-1)}\left(Y_{0} \cap B(x, \rho, \phi(n), g)\right) \cap B\left(f^{m(\phi(n)-1)}(x), \eta, r(n)+m, f\right)\right)
$$

which contains $f^{n}\left(B_{n}(x) \cap Y_{0}\right)$ by a number $N$ of sets $Y$ of volume $\leq 1$ with:

$$
N \leq C(X, l, r)^{\phi(n)+2 m} \prod_{0 \leq i \leq \phi(n)-1} \rho\left(g^{i}(x)\right)^{-2 l / r} \prod_{1 \leq i \leq m+r(n)} \eta\left(f^{n-i}(x)\right)^{-2 l / r} .
$$

Using now the fact that:

$$
\rho(y)=\left(\frac{d(y, I) \times \cdots \times d\left(f^{m-1}(y), I\right)}{K^{m}}\right)^{p},
$$

and

$$
\eta(y)=\left(\frac{d(y, I)}{K}\right)^{p}
$$

we have:

$$
\prod_{0 \leq i \leq \phi(n)-1} \rho\left(g^{i}(x)\right)^{-2 l / r} \leq K^{\frac{2 m \phi(n) p l}{r}} \prod_{0 \leq i \leq \phi(n) m-1} d\left(f^{i}(x), I\right)^{\frac{-2 p l}{r}},
$$

and

$$
\prod_{1 \leq i \leq m+r(n)} \eta\left(f^{n-i}(x)\right)^{-2 l / r} \leq K^{\frac{4 m p l}{r}} \prod_{1 \leq i \leq m+r(n)} d\left(f^{n-i}(x), I\right)^{\frac{-2 p l}{r}} .
$$

Finally, we have covered $f^{n}\left(B_{n}(x) \cap Y_{0}\right)$ by a number $N$ of sets $Y$ of volume $\leq 1$ with:

$$
N \leq C(X, l, r)^{n / m+2 m} K^{\frac{2 n p l}{r}+\frac{4 m p l}{r}} \prod_{0 \leq i \leq n-1} d\left(f^{i}(x), I\right)^{\frac{-4 p l}{r}} .
$$


That concludes the proof of Proposition 2.3.2.

2.3.4. Lower bound for the entropy of $\mu$. - Recall that we consider a cluster value $\mu$ of the sequence

$$
\mu_{n}=\frac{1}{n} \sum_{i=0}^{n-1} f_{*}^{i}\left(\frac{\left(f^{n}\right)^{*} \omega^{l} \wedge \omega^{k-l}}{\lambda_{l}\left(f^{n}\right)}\right)
$$

and that in order to simplify the notations we assume that $\left(\mu_{n}\right)$ converges to $\mu$. By assumption, $\mu$ gives no mass to the indeterminacy set $I$ and it is invariant by Lemma 2.1.3. The aim of this section is to prove that the metric entropy $h_{\mu}(f)$ is $\geq \log d_{l}-\delta$. This implies Theorem 1 by letting $\delta \rightarrow 0$.

So we have to bound $h_{\mu}(f)$. Here is the plan of this section: first we will construct partitions of finite entropy for $\mu$ that will be used latter with the proof of the variational principle to get the lower bound of the entropy that we want.

2.3.4.1. Construction of the partitions. - The proof is the one of Mañe (see Lemma 2 in [47]). We give his proof since we will use it in what follows. We consider a function $s(x)$ comprised between 0 and 1 . Later, we will take the values $\rho(x)$ or $\eta(x)$ for $s(x)$.

\section{Proposition 2.3.4. - (Mañé)}

We can construct a countable partition $\mathscr{P}$ of $X \backslash\{s=0\}$ such that:

1. If $x \in X \backslash\{s=0\}$, then diam $\mathscr{P}(x)<s(y)$ for all $y \in \mathscr{P}(x)$ (here $\mathscr{P}(x)$ denotes the atom of the partition that contains $x)$.

2. For any probability measure $\nu$ such that $\int \log s(x) d \nu(x)>-\infty$, we have $H_{\nu}(\mathscr{P})<+\infty$. Here $H_{\nu}(\mathscr{P})$ denotes the entropy of the partition $\mathscr{P}$ for the measure $\nu$.

Before proving the proposition, we give a multi-index version of Mañé's lemma (see Lemma 1 in [47]). We thank the referee for explaining to us this lemma which simplifies the proof of Proposition 2.3.8.

Lemma 2.3.5. - For all $q \in \mathbb{N}^{*}$, there exists a constant $C(q)$ such that:

For all family $\left(x_{s}\right)$ of real numbers $0 \leq x_{s} \leq 1$ indexed by $s=\left(s_{0}, \ldots, s_{q-1}\right) \in \mathbb{N}^{q}$ and for all $A \in \mathbb{N}$ we have:

$$
\sum_{|s| \geq A} x_{s} \log \left(1 / x_{s}\right) \leq \sum_{|s| \geq A}|s| x_{s}+C(q) e^{-\frac{A}{2 q}}
$$

with the convention that $x_{s} \log \left(1 / x_{s}\right)=0$ when $x_{s}=0$ and $|s|=s_{0}+\cdots+s_{q-1}$. 
Proof of Lemma 2.3.5. - The proof is the same that Mañe's one.

Let $\&$ be the set of multi-indexes $s \in \mathbb{N}^{q}$ for which $x_{s} \neq 0$ and $\log \left(1 / x_{s}\right) \leq|s|$. If $s \notin \varnothing$ then $x_{s} \leq e^{-|s|}$. Furthermore:

$$
\sum_{|s| \geq A} x_{s} \log \left(1 / x_{s}\right) \leq \sum_{|s| \geq A, s \in \varnothing}|s| x_{s}+\sum_{|s| \geq A, s \notin \phi}\left(\sqrt{x_{s}}\right)\left(\sqrt{x_{s}}\right) \log \left(1 / x_{s}\right) .
$$

But since $(\sqrt{t}) \log (1 / t) \leq 2 e^{-1}$ for all $t \geq 0$, we have:

$$
\sum_{|s| \geq A} x_{s} \log \left(1 / x_{s}\right) \leq \sum_{|s| \geq A}|s| x_{s}+2 e^{-1} \sum_{|s| \geq A, s \notin \phi} \sqrt{x_{s}}
$$

which is less than:

$$
\sum_{|s| \geq A}|s| x_{s}+2 e^{-1} \sum_{|s| \geq A} e^{-|s| / 2} .
$$

Now, $\{s,|s| \geq A\} \subset \cup_{i=0}^{q-1}\left\{s, s_{i} \geq A / q\right\}$, so $\sum_{|s| \geq A} e^{-|s| / 2}$ is less than

$$
\sum_{i=0}^{q-1} \sum_{\left\{s, s_{i} \geq A / q\right\}} e^{-s_{0} / 2-\cdots-s_{q-1} / 2} \leq q\left(\frac{1}{1-e^{-1 / 2}}\right)^{q} e^{-\frac{A}{2 q}} .
$$

That gives the lemma.

Proof of Proposition 2.3.4. - Here is Mañé's proof.

First of all, there are constants $C>0$ and $r_{0}>0$ such that for $0<r \leq r_{0}$, there exists a partition $\mathscr{P}_{r}$ of $X$ whose elements have a diameter $\leq r$ and such that the number of elements of the partition $\left|\mathscr{P}_{r}\right|$ is $\leq C(1 / r)^{2 k}$.

Now, we define $V_{n}:=\left\{x, e^{-(n+1)}<s(x) \leq e^{-n}\right\}$ for $n \geq 0$. Since the function $s$ is less than 1 , we have that $X \backslash\{s=0\}=\cup_{n \geq 0} V_{n}$.

Let $\mathscr{P}$ be the partition defined as follows: for $n$ fixed, we consider the sets $Q \cap V_{n}$ for $Q \in \mathscr{P}_{r_{n}}$ with $r_{n}=e^{-(n+1)}$. This defines a partition of $V_{n}$. Now, we get the partition $\mathscr{P}$ of $X \backslash\{s=0\}$ by taking all the $n$ between 0 and $+\infty$.

If $x \notin\{s=0\}$, then $x \in V_{n}$ for some $n \geq 0$ and then the atom $\mathscr{P}(x)$ of $\mathscr{P}$ containing $x$, is contained in an atom of $\mathscr{P}_{r_{n}}$, so we have:

$$
\operatorname{diam} \mathscr{P}(x) \leq e^{-(n+1)}<s(y)
$$

for all $y \in \mathscr{P}(x) \subset V_{n}$. This proves the first point of Proposition 2.3.4.

We now consider a measure $\nu$ such that $\int \log s(x) d \nu(x)>-\infty$. We want to show that $H_{\nu}(\mathscr{P})<+\infty$. We have:

$$
H_{\nu}(\mathscr{P})=\sum_{n=0}^{+\infty}\left(-\sum_{P \in \mathscr{D}, P \subset V_{n}} \nu(P) \log \nu(P)\right)
$$


Using the inequality:

$$
-\sum_{i=1}^{m_{0}} x_{i} \log x_{i} \leq\left(\sum_{i=1}^{m_{0}} x_{i}\right)\left(\log m_{0}-\log \sum_{i=1}^{m_{0}} x_{i}\right)
$$

which comes from the convexity of the function $\phi(x)=x \log (x)$ for $x \geq 0$, we get:

$$
H_{\nu}(\mathscr{P}) \leq \sum_{n=0}^{+\infty} \nu\left(V_{n}\right)\left(\log \left|\mathscr{P}_{r_{n}}\right|-\log \nu\left(V_{n}\right)\right)
$$

Since the number $\left|\mathscr{P}_{r_{n}}\right|$ of elements of $\mathscr{P}_{r_{n}}$ is less than $C e^{2 k(n+1)}$, we have:

$$
H_{\nu}(\mathscr{P}) \leq \log C+2 k \sum_{n=0}^{+\infty}(n+1) \nu\left(V_{n}\right)+\sum_{n=0}^{+\infty} \nu\left(V_{n}\right) \log \left(\frac{1}{\nu\left(V_{n}\right)}\right) .
$$

By assumption:

$$
\int \log s(x) d \nu(x)=\int_{\cup_{n \geq 0} V_{n}} \log s(x) d \nu(x)>-\infty .
$$

This implies that:

$$
\sum_{n=0}^{+\infty} n \nu\left(V_{n}\right)<+\infty
$$

and the proposition is then deduced from Lemma 2.3.5 with $q=1$ and $A=0$.

2.3.4.2. Lower bound for the entropy of $\mu$. - In what follows, we denote $\mathscr{P}$ (resp. Q) the partition previously constructed for $s(x)=\rho(x)(\operatorname{resp} . s(x)=\eta(x))$. Notice that $H_{\mu}(\mathscr{P})$ and $H_{\mu}(Q)$ are finite by using the previous Proposition since $\log d(x, I) \in$ $L^{1}(\mu)$ and $\mu$ is invariant. We consider the restriction of $\mathscr{P}$ and $Q$ to $\Omega=X \backslash \cup_{i \geq 0} f^{-i}(I)$ (that we still denote $\mathscr{P}$ and $Q$ ). They are partitions of $\Omega$. The advantage of those partitions over $\Omega$ is that the $f^{i}$ are well-defined on them. In particular, we can define for example the partition $f^{-i}(\mathscr{P})$ : its atoms are the $f^{-i}(P):=\left\{x \in \Omega\right.$ with $\left.f^{i}(x) \in P\right\}$ where the $P$ are the atoms of $\mathscr{P}$. Since $f(\Omega) \subset \Omega$, we get a partition of $\Omega$. The measures that we consider $\left(\nu_{n}, \mu_{n}\right.$ or $\left.\mu\right)$ have mass 1 on $\Omega$. The parts of $X$ that we drop are of mass 0 for them. We remark that with our convention, we have: $f^{-a}\left(f^{-b}(P)\right)=f^{-a-b}(P)=\left\{x \in \Omega\right.$ with $\left.f^{a+b}(x) \in P\right\}$. Recall that we denote:

$$
\nu_{n}=\frac{\left(f^{n}\right)^{*} \omega^{l} \wedge \omega^{k-l}}{\lambda_{l}\left(f^{n}\right)}
$$

and that $\nu_{n}\left(A_{n}\right) \geq 1-\frac{1}{C_{0}}($ see Lemma 2.3.1).

In what follows, we denote $\nu_{n}^{\prime}:=\frac{\nu_{n} \mid A_{n}}{\nu_{n}\left(A_{n}\right)}$ (i.e. $\left.\nu_{n}^{\prime}(B)=\frac{\nu_{n}\left(B \cap A_{n}\right)}{\nu_{n}\left(A_{n}\right)}\right)$.

Define the joint partition $\mathscr{P}_{-n}$ of the partitions $\mathscr{P}$ and $Q$ by (recall that $n=$ $\phi(n) m+r(n)$ with $0 \leq r(n)<m)$ :

$$
\mathscr{P}_{-n}:=\mathscr{P} \vee f^{-1}(\mathscr{P}) \vee \cdots \vee f^{-\phi(n) m+m}(\mathscr{P}) \vee f^{-\phi(n) m+m-1}(Q) \vee \cdots \vee f^{-n+1}(Q) .
$$


First, we have the lemma:

LEMMA 2.3.6. - If $n$ is large enough, then

$$
\nu_{n}^{\prime}\left(\mathscr{P}_{-n}(x)\right) \leq \frac{e^{10 \delta n l p}}{\lambda_{l}\left(f^{n}\right)} \frac{1}{1-\frac{1}{C_{0}}} .
$$

for every atom $\mathscr{P}_{-n}(x)$ of $\mathscr{P}_{-n}$.

Proof. - Recall that:

$$
B_{n}(x)=B\left(x, \rho, \phi(n), f^{m}\right) \cap f^{-\phi(n) m+m}\left(B\left(f^{\phi(n) m-m}(x), \eta, r(n)+m, f\right)\right) .
$$

We have shown in the previous paragraph that if $n$ is large enough then for every $x \in A_{n}(\operatorname{see}(2))$ :

$$
\nu_{n}\left(B_{n}(x)\right) \leq \frac{e^{10 \delta n p l}}{\lambda_{l}\left(f^{n}\right)} .
$$

Let $n$ be large enough so that the previous property is satisfied. If $\mathscr{P}_{-n}(x)$ does not contain any points of $A_{n}$ then $\nu_{n}^{\prime}\left(\mathscr{P}_{-n}(x)\right)=0$ and the lemma is true. So we can assume that there exists $y \in \mathscr{P}_{-n}(x) \cap A_{n}$.

By definition of the joint partition, we have $\mathscr{P}_{-n}(x)$ which is equal to:

$$
\begin{aligned}
& \mathscr{P}(x) \cap \cdots \cap f^{-\phi(n) m+m}\left(\mathscr{P}\left(f^{\phi(n) m-m}(x)\right)\right) \\
& \cap f^{-\phi(n) m+m-1}\left(Q\left(f^{\phi(n) m-m+1}(x)\right)\right) \cap \cdots \cap f^{-n+1}\left(Q\left(f^{n-1}(x)\right)\right) .
\end{aligned}
$$

In particular, $f^{i}(y) \in \mathscr{P}\left(f^{i}(x)\right)$ for $i=0 \ldots \phi(n) m-m$ and then $f^{i}(y) \in Q\left(f^{i}(x)\right)$ for $i=\phi(n) m-m+1 \ldots n-1$. By Proposition 2.3.4, the diameter of $\mathscr{P}\left(f^{i}(x)\right)$ is $\leq \rho\left(f^{i}(y)\right)$ for $i=0 \ldots \phi(n) m-m$ and the diameter of $Q\left(f^{i}(x)\right)$ is $\leq \eta\left(f^{i}(y)\right)$ for $i=\phi(n) m-m+1 \ldots n-1$ which means:

$$
\mathscr{P}_{-n}(x) \subset B_{n}(y) .
$$

The lemma follows then first from the estimation of the previous paragraph since $y \in A_{n}$ and secondly from the fact that $\nu_{n}\left(A_{n}\right)$ is $\geq 1-\frac{1}{C_{0}}$.

Thanks to this estimation on $\nu_{n}^{\prime}\left(\mathscr{P}_{-n}(x)\right)$, we can bound the entropy of $\mu$ using a variation of the proof of the variational principle. We refer the reader to [54] p.188-190 for the proof of the principle and to [4], [10] or [42] for its use to bound from below the entropies of measures in holomorphic or meromorphic dynamics.

Let $q$ be an integer $2 m<q<n$ (with $m$ from the above paragraph). For $0 \leq j \leq$ $q-1$, we let $a(j)=\left[\frac{n-j}{q}\right]$ and then

$$
\{0,1, \ldots, n-1\}=\{j+r q+i, 0 \leq r \leq a(j)-2,0 \leq i \leq q-1\} \cup S(j)
$$

where $S(j)=\{0,1, \ldots, j-1, j+(a(j)-1) q, j+(a(j)-1) q+1, \ldots, n-1\}$ is of cardinality less than $3 q$ since $j+(a(j)-1) q \geq j+\left(\frac{n-j}{q}-2\right) q=n-2 q$. We took the indexes $r$ up to $a(j)-2$ so that $S(j)$ contains $n-q \ldots n-1$ and so in particular $\phi(n) m-m+1 \ldots n-1$ 
(we take $q$ large with respect to $m$ ). We denote $S_{1}(j)$ the elements of $S(j)$ other than $\phi(n) m-m+1 \ldots n-1$ and $S_{2}(j)$ the elements $\phi(n) m-m+1 \ldots n-1$.

Now, we have (see for example Proposition 4.3.3 of [45]):

$$
H_{\nu_{n}^{\prime}}\left(\mathscr{P}_{-n}\right) \geq-\log \left(\sup _{P \in \mathscr{P}_{-n}} \nu_{n}^{\prime}(P)\right) \geq-10 \delta n l p+\log \lambda_{l}\left(f^{n}\right)+\log \left(1-\frac{1}{C_{0}}\right),
$$

by the previous lemma.

On the other hand, by the proof of the variational principle for $0 \leq j \leq q-1$, we have:

$$
\mathscr{P}_{-n}=\bigvee_{r=0}^{a(j)-2}\left(f^{-(r q+j)} \bigvee_{i=0}^{q-1} f^{-i} \mathscr{P}\right) \vee \bigvee_{t \in S_{1}(j)} f^{-t} \not \mathcal{P} \vee \bigvee_{t \in S_{2}(j)} f^{-t} Q
$$

So, (again by Proposition 4.3.3 in [45]):

$$
H_{\nu_{n}^{\prime}}\left(\mathscr{P}_{-n}\right) \leq \sum_{r=0}^{a(j)-2} H_{\nu_{n}^{\prime}}\left(f^{-(r q+j)} \bigvee_{i=0}^{q-1} f^{-i} \mathscr{P}\right)+\sum_{t \in S_{1}(j)} H_{\nu_{n}^{\prime}}\left(f^{-t} \mathscr{P}\right)+\sum_{t \in S_{2}(j)} H_{\nu_{n}^{\prime}}\left(f^{-t} Q\right)
$$

which is equal to:

$$
\sum_{r=0}^{a(j)-2} H_{f_{*}^{r q+j} \nu_{n}^{\prime}}\left(\bigvee_{i=0}^{q-1} f^{-i} \mathscr{P}\right)+\sum_{t \in S_{1}(j)} H_{\nu_{n}^{\prime}}\left(f^{-t} \mathscr{P}\right)+\sum_{t \in S_{2}(j)} H_{\nu_{n}^{\prime}}\left(f^{-t} \mathcal{Q}\right)
$$

Summing this relation for $j=0 \ldots q-1$, we get:

$$
\begin{aligned}
& q\left(-10 \delta n l p+\log \lambda_{l}\left(f^{n}\right)+\log \left(1-\frac{1}{C_{0}}\right)\right) \\
& \leq \sum_{j=0}^{q-1} \sum_{r=0}^{a(j)-2} H_{f_{*}^{r+j} \nu_{n}^{\prime}}\left(\bigvee_{i=0}^{q-1} f^{-i} \mathscr{P}\right)+\sum_{j=0}^{q-1}\left(\sum_{t \in S_{1}(j)} H_{\nu_{n}^{\prime}}\left(f^{-t} \mathscr{P}\right)+\sum_{t \in S_{2}(j)} H_{\nu_{n}^{\prime}}\left(f^{-t} Q\right)\right) .
\end{aligned}
$$

The integers $j+r q$ for $0 \leq j \leq q-1$ and $0 \leq r \leq a(j)-2$ are all distinct and $\leq n-2 q$.

So we have that (using the convexity of the function $\Phi(x)=x \log (x)$ for $x>0$ ):

$$
\text { (I): } \frac{q}{n-2 q+1}\left(-10 \delta n l p+\log \lambda_{l}\left(f^{n}\right)+\log \left(1-\frac{1}{C_{0}}\right)\right)
$$

which is less than:

$$
H_{\frac{1}{n-2 q+1} \sum_{p=0}^{n-2 q} f_{*}^{p} \nu_{n}^{\prime}}\left(\bigvee_{i=0}^{q-1} f^{-i} \mathscr{P}\right)+\sum_{j=0}^{q-1}\left(\sum_{t \in S_{1}(j)} \frac{H_{\nu_{n}^{\prime}}\left(f^{-t} \mathscr{P}\right)}{n-2 q+1}+\sum_{t \in S_{2}(j)} \frac{H_{\nu_{n}^{\prime}}\left(f^{-t} Q\right)}{n-2 q+1}\right) .
$$

Here is the plan of the rest of the proof. In a first time, we deduce from that inequality a lower bound of $\frac{1}{q} H_{\frac{1}{n-2 q+1}} \sum_{p=0}^{n-2 q} f_{*}^{p} \nu_{n}\left(\bigvee_{i=0}^{q-1} f^{-i \not \mathcal{P}}\right)$. Then we will pass to the limit in that inequality. 
1) Lower bound of $\frac{1}{q} H_{\frac{1}{n-2 q+1}} \sum_{p=0}^{n-2 q} f_{*}^{p} \nu_{n}\left(\bigvee_{i=0}^{q-1} f^{-i \not \mathcal{P}}\right)$. - By definition, $\nu_{n}^{\prime}:=\frac{\nu_{n} \mid A_{n}}{\nu_{n}\left(A_{n}\right)}$. In particular, $\nu_{n}^{\prime} \leq \frac{\nu_{n}}{1-\frac{1}{C_{0}}}$ and

$$
\frac{1}{n-2 q+1} \sum_{p=0}^{n-2 q} f_{*}^{p} \nu_{n}^{\prime} \leq \frac{1}{\left(1-\frac{1}{C_{0}}\right)(n-2 q+1)} \sum_{p=0}^{n-2 q} f_{*}^{p} \nu_{n} .
$$

In order to control $\frac{1}{q} H_{\frac{1}{n-2 q+1}} \sum_{p=0}^{n-2 q} f_{*}^{p} \nu_{n}\left(\bigvee_{i=0}^{q-1} f^{-i} \mathscr{P}\right)$ with the inequality (I), we are going to use the following lemma:

LEMMA 2.3.7. - Let $\nu$ and $\nu^{\prime}$ be two probabilities such that $\nu^{\prime} \leq \beta \nu$ for some $\beta>1$. Then for any partition $Q$, we have:

$$
H_{\nu^{\prime}}(Q) \leq \beta\left(H_{\nu}(Q)+1\right) .
$$

We assume temporarily this lemma and we continue the proof.

Now, since:

$$
\nu_{n}^{\prime} \leq \beta \nu_{n}
$$

with $\beta=\frac{1}{1-\frac{1}{C_{0}}}$, the previous lemma gives that (I) is less than

$$
\begin{aligned}
& \frac{1}{1-\frac{1}{C_{0}}}\left[H_{\frac{1}{n-2 q+1}} \sum_{p=0}^{n-2 q} f_{*}^{p} \nu_{n}\left(\bigvee_{i=0}^{q-1} f^{-i} \mathscr{P}\right)+1\right. \\
& \left.+\sum_{j=0}^{q-1}\left(\sum_{t \in S_{1}(j)} \frac{H_{\nu_{n}}\left(f^{-t} \mathscr{P}\right)}{n-2 q+1}+\sum_{t \in S_{2}(j)} \frac{H_{\nu_{n}}\left(f^{-t} Q\right)}{n-2 q+1}\right)+\frac{3 q^{2}}{n-2 q+1}\right]
\end{aligned}
$$

(since the cardinality of $S(j)$ is $\leq 3 q$ ).

This implies a lower bound of $\frac{1}{q} H_{\frac{1}{n-2 q+1}} \sum_{p=0}^{n-2 q} f_{*}^{p} \nu_{n}\left(\bigvee_{i=0}^{q-1} f^{-i} \mathscr{P}\right)$ by

$$
\begin{aligned}
& \left(1-\frac{1}{C_{0}}\right)\left(\frac{1}{n-2 q+1}\left(-10 \delta n l p+\log \lambda_{l}\left(f^{n}\right)+\log \left(1-\frac{1}{C_{0}}\right)\right)\right) \\
& -\frac{1}{q}-\frac{1}{q}\left(\sum_{j=0}^{q-1} \sum_{t \in S_{1}(j)} \frac{H_{\nu_{n}}\left(f^{-t} \not \mathcal{P}\right)}{n-2 q+1}+\sum_{j=0}^{q-1} \sum_{t \in S_{2}(j)} \frac{H_{\nu_{n}}\left(f^{-t} Q\right)}{n-2 q+1}\right)-\frac{3 q}{n-2 q+1} .
\end{aligned}
$$

It remains now to take the limit of that inequality when $n$ goes to $\infty$.

2) Pass to the limit $n \rightarrow+\infty$. - First:

$$
\frac{1}{n-2 q+1}\left(-10 \delta n l p+\log \lambda_{l}\left(f^{n}\right)+\log \left(1-\frac{1}{C_{0}}\right)\right)
$$

goes to $-10 \delta l p+\log d_{l}$ when $n \rightarrow \infty$. Now, we need the following proposition.

Proposition 2.3.8. - We have: 
1. For all $q>2 m$,

$$
H_{\frac{1}{n-2 q+1}} \sum_{p=0}^{n-2 q} f_{*}^{p} \nu_{n}\left(\bigvee_{i=0}^{q-1} f^{-i} \not \mathcal{P}\right)
$$

converges to $H_{\mu}\left(\bigvee_{i=0}^{q-1} f^{-i} \not \mathcal{P}\right)$ when $n \rightarrow \infty$.

2. For $q>2 m$ :

$$
\frac{1}{q} \sum_{j=0}^{q-1} \sum_{t \in S_{1}(j)} \frac{H_{\nu_{n}}\left(f^{-t} \not \mathscr{P}\right)}{n-2 q+1}
$$

converges to 0 when $n \rightarrow \infty$.

3. For $q>2 m$ :

$$
\frac{1}{q} \sum_{j=0}^{q-1} \sum_{t \in S_{2}(j)} \frac{H_{\nu_{n}}\left(f^{-t} Q\right)}{n-2 q+1}
$$

converges to 0 when $n \rightarrow \infty$.

End of the proof of Theorem 1. - We assume temporarily that the proposition is true. We finish the lower bound of the entropy of $\mu$.

If we pass to the limit in the inequality of the previous paragraph, we get:

$$
\frac{1}{q} H_{\mu}\left(\bigvee_{i=0}^{q-1} f^{-i} \not \mathscr{P}\right) \geq\left(1-\frac{1}{C_{0}}\right)\left(-10 \delta l p+\log d_{l}\right)-\frac{1}{q}
$$

If we let $q$ go to $\infty$, we have:

$$
h_{\mu}(f) \geq\left(1-\frac{1}{C_{0}}\right)\left(-10 \delta l p+\log d_{l}\right)
$$

because the entropy of $\mathscr{P}$ is finite for $\mu$.

This proves the theorem by letting $C_{0}$ go to $\infty$ then by letting $\delta$ go to 0 .

Up to the proof of Lemma 2.3.7 and Proposition 2.3.8, we have proved Theorem 1.

Proof of Lemma 2.3.7. - The function $\Phi(x)=-x \log (x)$ is increasing on $\left[0, e^{-1}\right]$ and decreasing on $\left[e^{-1}, 1\right]$. So we have:

$$
\begin{aligned}
H_{\nu^{\prime}}(Q) & =\sum_{Q \in Q}-\nu^{\prime}(Q) \log \nu^{\prime}(Q) \\
& =\sum_{Q \in \mathcal{Q}, \nu(Q) \leq \frac{e^{-1}}{\beta}}-\nu^{\prime}(Q) \log \nu^{\prime}(Q)+\sum_{Q \in Q, \nu(Q)>\frac{e^{-1}}{\beta}}-\nu^{\prime}(Q) \log \nu^{\prime}(Q)
\end{aligned}
$$

which is less than:

$$
\sum_{Q \in Q, \nu(Q) \leq \frac{e^{-1}}{\beta}}-\beta \nu(Q) \log (\beta \nu(Q))+\sum_{Q \in \mathcal{Q}, \nu(Q)>\frac{e^{-1}}{\beta}}-\nu^{\prime}(Q) \log \nu^{\prime}(Q) .
$$


Since they are at most $\frac{\beta}{e^{-1}}$ of $Q \in Q$ with $\nu(Q)>\frac{e^{-1}}{\beta}$ and because on the interval $[0,1]$, the function $\Phi(x)$ is non negative and bounded by $e^{-1}$, we have:

$$
H_{\nu^{\prime}}(Q) \leq \beta H_{\nu}(Q)+\beta
$$

Proof of Proposition 2.3.8. - In order to simplify the notations, we denote:

$$
\mu_{n}^{\prime}=\frac{1}{n-2 q+1} \sum_{p=0}^{n-2 q} f_{*}^{p} \nu_{n}
$$

For the proof of the three points of the proposition, we will use the following lemma:

LemMA 2.3.9. - For $i, j=0 \ldots q-1$, we have

$$
0 \geq \int_{\left\{\rho \circ f^{i} \leq \epsilon\right\}} \log \rho \circ f^{j} d \mu_{n}^{\prime} \geq-\delta(\epsilon)
$$

if $n$ is large enough. Here $\delta(\epsilon)$ goes to 0 when $\epsilon$ goes to 0 .

Moreover, we have the same lemma with $\mu$ instead of $\mu_{n}^{\prime}$.

Proof. - We give the proof for $\mu_{n}^{\prime}$. It is the same for $\mu$.

First step. - We show first that for $j=0 \ldots 2 q-1$, we have

$$
0 \geq \int_{\left\{d\left(f^{j}(x), I\right) \leq \epsilon\right\}} \log d\left(f^{j}(x), I\right) d \mu_{n}^{\prime}(x) \geq-\delta^{\prime}(\epsilon)
$$

for $n$ large enough. Here $\delta^{\prime}(\epsilon)$ goes to 0 when $\epsilon$ goes to 0 .

The last integral is equal to $A=\int_{\{d(x, I) \leq \epsilon\}} \log d(x, I) d\left(\left(f^{j}\right)_{*} \mu_{n}^{\prime}\right)(x)$. But $\left(f^{j}\right)_{*} \mu_{n}^{\prime}$ is smaller that $\frac{n}{n-2 q+1} \mu_{n}$ for $j=0 \ldots 2 q-1$. So, we have

$$
A \geq \frac{n}{n-2 q+1} \int_{\{d(x, I) \leq \epsilon\}} \log d(x, I) d \mu_{n}(x) .
$$

Now, $\int_{\{d(x, I) \leq \epsilon\}} \log d(x, I) d \mu_{n}(x)$ converges to $\int_{\{d(x, I) \leq \epsilon\}} \log d(x, I) d \mu(x)$. Indeed, on one hand we have that:

$$
\int \log d(x, I) d \mu_{n}(x)
$$

converges to $\int \log d(x, I) d \mu(x)$ by Hypothesis $(\mathrm{H})$. On the other hand:

$$
\int_{\{d(x, I)>\epsilon\}} \log d(x, I) d \mu_{n}(x)
$$

converges to $\int_{\{d(x, I)>\epsilon\}} \log d(x, I) d \mu(x)$ up to choosing $\epsilon$ generic so that $\mu$ gives no mass to $\{x, d(x, I)=\epsilon\}$.

Finally, since $\int_{\{d(x, I) \leq \epsilon\}} \log d(x, I) d \mu(x)$ goes to 0 when $\epsilon$ converges to 0 by dominated convergence, the first step follows. 
Second step. - Here, we prove that for $i, j=0 \ldots 2 q-1$, we have

$$
0 \geq \int_{\left\{d\left(f^{i}(x), I\right) \leq \epsilon\right\}} \log d\left(f^{j}(x), I\right) d \mu_{n}^{\prime}(x) \geq-\delta^{\prime \prime}(\epsilon)
$$

for $n$ large enough. Here $\delta^{\prime \prime}(\epsilon)$ goes to 0 when $\epsilon$ goes to 0 .

For that, we split this integral into two parts:

$$
\begin{aligned}
& \int_{\left\{d\left(f^{i}(x), I\right) \leq \epsilon\right\} \cap\left\{d\left(f^{j}(x), I\right) \leq \delta^{\prime}(\epsilon)\right\}} \log d\left(f^{j}(x), I\right) d \mu_{n}^{\prime}(x) \\
& +\int_{\left\{d\left(f^{i}(x), I\right) \leq \epsilon\right\} \cap\left\{d\left(f^{j}(x), I\right)>\delta^{\prime}(\epsilon)\right\}} \log d\left(f^{j}(x), I\right) d \mu_{n}^{\prime}(x) .
\end{aligned}
$$

The first term is larger than

$$
\int_{\left\{d\left(f^{j}(x), I\right) \leq \delta^{\prime}(\epsilon)\right\}} \log d\left(f^{j}(x), I\right) d \mu_{n}^{\prime}(x)
$$

and this term is bounded by below by $-\delta^{\prime}\left(\delta^{\prime}(\epsilon)\right)$ if $n$ is large enough by using the first step. That quantity goes to 0 when $\epsilon$ goes to 0 .

The second term is larger than (if $\epsilon \leq e^{-1}$ and $\delta^{\prime}(\epsilon) \leq 1$ )

$$
\begin{aligned}
& \int_{\left\{d\left(f^{i}(x), I\right) \leq \epsilon\right\}} \log \delta^{\prime}(\epsilon) d \mu_{n}^{\prime}(x) \\
& \geq-\log \delta^{\prime}(\epsilon) \int_{\left\{d\left(f^{i}(x), I\right) \leq \epsilon\right\}} \log d\left(f^{i}(x), I\right) d \mu_{n}^{\prime}(x) \geq \delta^{\prime}(\epsilon) \log \delta^{\prime}(\epsilon)
\end{aligned}
$$

if $n$ is large enough by using the first step. That quantity goes to 0 when $\epsilon$ goes to 0 .

Third step. - Now, we prove the lemma. Recall that:

$$
\rho(x)=\left(\frac{d(x, I) \times \cdots \times d\left(f^{m-1}(x), I\right)}{K^{m}}\right)^{p} .
$$

In particular, we have:

$$
\left\{x, \rho \circ f^{i}(x) \leq \epsilon\right\} \subset\left\{x, d\left(f^{i}(x), I\right) \leq \epsilon^{\frac{1}{m p}} K\right\} \cup \cdots \cup\left\{x, d\left(f^{i+m-1}(x), I\right) \leq \epsilon^{\frac{1}{m p}} K\right\} .
$$

So, for $i, j=0 \ldots q-1$, we have

$$
0 \geq \int_{\left\{\rho \circ f^{i} \leq \epsilon\right\}} \log \rho \circ f^{j} d \mu_{n}^{\prime}
$$

which is larger than

$$
\sum_{l=0}^{m-1} \int_{\left\{d\left(f^{l+i}(x), I\right) \leq \epsilon^{\frac{1}{m p}} K\right\}} \log \rho \circ f^{j} d \mu_{n}^{\prime}
$$


Now, for $l$ between 0 and $m-1$,

$$
\begin{aligned}
& \int_{\left\{d\left(f^{l+i}(x), I\right) \leq \epsilon^{\frac{1}{m p}} K\right\}} \log \rho \circ f^{j} d \mu_{n}^{\prime}= \\
& \sum_{s=0}^{m-1}\left(p \int_{\left\{d\left(f^{l+i}(x), I\right) \leq \epsilon^{\frac{1}{m p}} K\right\}} \log d\left(f^{s+j}(x), I\right) d \mu_{n}^{\prime}(x)\right. \\
& \left.-p \log K \int_{\left\{d\left(f^{l+i}(x), I\right) \leq \epsilon^{\frac{1}{m p}} K\right\}} d \mu_{n}^{\prime}(x)\right) \\
& \geq-m p \delta^{\prime \prime}\left(\epsilon^{\frac{1}{m p}} K\right)+m p \log K \int_{\left\{d\left(f^{l+i}(x), I\right) \leq \epsilon^{\frac{1}{m p}} K\right\}} \log d\left(f^{l+i}(x), I\right) d \mu_{n}^{\prime}(x)
\end{aligned}
$$

for $\epsilon$ small enough so that $\epsilon^{\frac{1}{m p}} K \leq e^{-1}$ and $n$ large enough by using the second step (recall that $m<q$ ).

Finally this term is larger than $-2 m p \log K \delta^{\prime \prime}\left(\epsilon^{\frac{1}{m p}} K\right)$ for $n$ large enough and it was the inequality that we were looking for.

First point of the proposition 2.3.8. - We thank the referee for the simplification of the proof of this point.

We recall that in this paragraph, we consider the partition $\mathscr{P}$ which is constructed as in Proposition 2.3.4 with $s(x)=\rho(x)$. In particular, we have $V_{j}:=\left\{x, e^{-(j+1)}<\right.$ $\left.\rho(x) \leq e^{-j}\right\}$ for $j \in \mathbb{N}$.

For a multi-index $s=\left(s_{0}, \ldots, s_{q-1}\right) \in \mathbb{N}^{q}$, we denote by $W_{s}=V_{s_{0}} \cap \cdots \cap f^{-q+1} V_{s_{q-1}}$. When $P \in \bigvee_{i=0}^{q-1} f^{-i} \mathscr{P}$, then $P$ is in some $W_{s}$ for some multi-index $s$. Moreover, the number of $P \in \bigvee_{i=0}^{q-1} f^{-i} \mathscr{P}$ which are in $W_{s}$ is bounded from above by $C^{q} e^{2 k q} e^{2 k|s|}=$ $C_{0}^{q} e^{2 k|s|}$ with $|s|=s_{0}+\cdots+s_{q-1}$.

Now, for $A \in \mathbb{N}^{*}$, we have

$$
H_{\mu_{n}^{\prime}}\left(\bigvee_{i=0}^{q-1} f^{-i} \mathscr{P}\right)=\sum_{s \in \mathbb{N}^{q}} \sum_{P \in \bigvee_{i=0}^{q-1}}-\mu_{n}^{\prime}(P) \log \mu_{n}^{\prime}(P)
$$

that we divide as:

$$
\sum_{|s| \geq A} \sum_{\substack{P \in \bigvee_{i=0}^{q-1} \\ f^{-i} \mathscr{\mathcal { D }}, P \subset W_{s}}}-\mu_{n}^{\prime}(P) \log \mu_{n}^{\prime}(P)+\sum_{|s|<A} \sum_{P \in \bigvee_{i=0}^{q-1} f^{-i} \mathscr{P}, P \subset W_{s}}-\mu_{n}^{\prime}(P) \log \mu_{n}^{\prime}(P) .
$$


We begin with the first term. We use the notations and the ideas of the proof of Proposition 2.3.4.

$$
\begin{aligned}
& R_{1}(A)=\sum_{|s| \geq A} \sum_{P \in \bigvee_{i=0}^{q-1}} \sum_{f^{-i} \mathscr{P}, P \subset W_{s}}-\mu_{n}^{\prime}(P) \log \mu_{n}^{\prime}(P) \\
& \leq \sum_{|s| \geq A} \mu_{n}^{\prime}\left(W_{s}\right)\left(\log \left(\#\left\{P \in \bigvee_{i=0}^{q-1} f^{-i} \mathscr{P}, P \subset W_{s}\right\}\right)-\log \mu_{n}^{\prime}\left(W_{s}\right)\right) \\
& \leq \log C_{0}^{q} \sum_{|s| \geq A} \mu_{n}^{\prime}\left(W_{s}\right)+2 k \sum_{|s| \geq A}|s| \mu_{n}^{\prime}\left(W_{s}\right)+\sum_{|s| \geq A} \mu_{n}^{\prime}\left(W_{s}\right) \log \frac{1}{\mu_{n}^{\prime}\left(W_{s}\right)} .
\end{aligned}
$$

Now, by using Lemma 2.3.5, this is less than

$$
C(q) e^{-\frac{A}{2 q}}+\left(\log C_{0}^{q}+2 k+1\right) \sum_{|s| \geq A}|s| \mu_{n}^{\prime}\left(W_{s}\right) .
$$

On $W_{s}$, we have $-\sum_{j=0}^{q-1} \log \rho \circ f^{j} \geq|s|$. Moreover, the $W_{s}$ are pairwise disjoints and

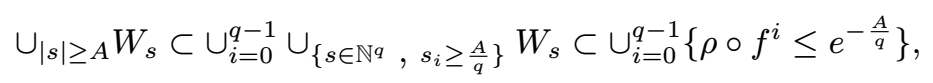

so,

$$
\begin{aligned}
& R_{1}(A) \leq C(q) e^{-\frac{A}{2 q}}+\left(\log C_{0}^{q}+2 k+1\right) \sum_{j=0}^{q-1} \sum_{|s| \geq A} \int_{W_{s}}-\log \rho \circ f^{j}(x) d \mu_{n}^{\prime}(x) \\
& \leq C(q) e^{-\frac{A}{2 q}}+\left(\log C_{0}^{q}+2 k+1\right) \sum_{j=0}^{q-1} \sum_{i=0}^{q-1} \int_{\left\{\rho \circ f^{i}(x) \leq e^{-\frac{A}{q}}\right\}}-\log \rho \circ f^{j}(x) d \mu_{n}^{\prime}(x)
\end{aligned}
$$

which is as small that we want if we take $A$ large and then $n$ large enough by using the previous lemma. The same thing is true if we replace $\mu_{n}^{\prime}$ by $\mu$ in $R_{1}(A)$ : it is as small as we want if we take $A$ large enough by using the previous lemma.

We now consider the second term

$$
\sum_{|s|<A} \sum_{P \in \bigvee_{i=0}^{q-1}}-\mu_{n}^{\prime-i \not \mathcal{P}}, P \subset W_{s}(P) \log \mu_{n}^{\prime}(P) .
$$

Up to moving slightly the boundaries of the partition $\mathscr{P}$, we can assume that $\mu$ gives no mass to the boundary of its elements. In particular, the above term converges to:

$$
\sum_{|s|<A} \sum_{P \in \bigvee_{i=0}^{q-1}}-\mu(P) \log \mu(P),
$$

when $n$ goes to infinity since we only consider a finite number of elements and $\mu_{n}^{\prime}$ converges to $\mu$.

In particular, this implies that $H_{\mu_{n}^{\prime}}\left(\bigvee_{i=0}^{q-1} f^{-i} \mathscr{P}\right)$ converges to $H_{\mu}\left(\bigvee_{i=0}^{q-1} f^{-i} \mathscr{P}\right)$. 
Second point of Proposition 2.3.8. - We show that:

$$
\frac{1}{q} \sum_{j=0}^{q-1} \sum_{t \in S_{1}(j)} \frac{H_{\nu_{n}}\left(f^{-t} \mathscr{P}\right)}{n-2 q+1}
$$

converges to 0 when $n$ goes to $\infty$.

First, we consider $H_{\nu_{n}}\left(f^{-t} \mathscr{P}\right)$. We have

$$
H_{\nu_{n}}\left(f^{-t} \mathscr{P}\right)=\sum_{s=0}^{+\infty}-\sum_{P \in \mathscr{P}, P \subset V_{s}}\left(\left(f^{t}\right)_{*} \nu_{n}\right)(P) \log \left(\left(f^{t}\right)_{*} \nu_{n}\right)(P)
$$

which is less than (see the proof of Proposition 2.3.4)

$$
\begin{aligned}
& \sum_{s=0}^{+\infty}\left(\left(f^{t}\right)_{*} \nu_{n}\right)\left(V_{s}\right)\left(\log \left|\mathscr{P}_{r_{s}}\right|-\log \left(\left(f^{t}\right)_{*} \nu_{n}\right)\left(V_{s}\right)\right) \\
& \leq \log C+2 k \sum_{s=0}^{+\infty}(s+1)\left(\left(f^{t}\right)_{*} \nu_{n}\right)\left(V_{s}\right)+\sum_{s=0}^{+\infty}\left(\left(f^{t}\right)_{*} \nu_{n}\right)\left(V_{s}\right) \log \frac{1}{\left(\left(f^{t}\right)_{*} \nu_{n}\right)\left(V_{s}\right)} \\
& \leq \log C+2 k+(2 k+1) \sum_{s=0}^{+\infty} s\left(\left(f^{t}\right)_{*} \nu_{n}\right)\left(V_{s}\right)+C(1) \\
& =\log C+2 k+(2 k+1) \sum_{s=0}^{s_{0}-1} s\left(\left(f^{t}\right)_{*} \nu_{n}\right)\left(V_{s}\right)+(2 k+1) \sum_{s=s_{0}}^{+\infty} s\left(\left(f^{t}\right)_{*} \nu_{n}\right)\left(V_{s}\right)+C(1)
\end{aligned}
$$

The last inequality comes from Lemma 2.3 .5 with $q=1$ and $A=0$.

So, to prove the second point, it is sufficient to show that

$$
\frac{1}{q} \sum_{j=0}^{q-1} \sum_{t \in S_{1}(j)} \frac{\sum_{s=s_{0}}^{+\infty} s\left(\left(f^{t}\right)_{*} \nu_{n}\right)\left(V_{s}\right)}{n-2 q+1}
$$

is as small as we want for $s_{0}$ large and then $n$ large enough because the cardinality of $S_{1}(j)$ is smaller than $3 q$.

This last term is less than

$$
-\frac{1}{q(n-2 q+1)} \sum_{j=0}^{q-1} \sum_{t \in S_{1}(j)} \int_{\left\{\rho \leq e^{-s_{0}}\right\}} \log \rho d\left(f^{t}\right)_{*} \nu_{n} .
$$

But:

$$
\sum_{t \in S_{1}(j)}\left(f^{t}\right)_{*} \nu_{n} \leq(\phi(n) m-m+1) \mu_{n}^{\prime \prime}
$$

with $\mu_{n}^{\prime \prime}=\frac{1}{\phi(n) m-m+1} \sum_{p=0}^{\phi(n) m-m} f_{*}^{p} \nu_{n}$. 
Following Lemma 2.3.9 with $\mu_{n}^{\prime \prime}$ instead of $\mu_{n}^{\prime}$ and $i=j=0$ (this is indeed possible because the indexes $p$ in $\mu_{n}^{\prime \prime}$ goes to $\phi(n) m-m$ which is $\left.\leq n-1-(m-1)\right)$, we deduce that:

$$
\frac{1}{q(n-2 q+1)} \sum_{j=0}^{q-1} \sum_{t \in S_{1}(j)} \sum_{s=s_{0}}^{+\infty} s\left(\left(f^{t}\right)_{*} \nu_{n}\right)\left(V_{s}\right)
$$

is as small as we want by taking $s_{0}$ large enough and then $n$ large enough.

This gives the second point of Proposition 2.3.8.

Third point of Proposition 2.3.8. - The proof is the same than for the second point by replacing $S_{1}(j)$ by $S_{2}(j), \mathscr{P}$ by $Q$ and $\rho$ by $\eta$.

At the end, we have to bound from above:

$$
\frac{1}{q(n-2 q+1)} \sum_{j=0}^{q-1} \sum_{t \in S_{2}(j)} \sum_{s=s_{0}}^{+\infty} s\left(\left(f^{t}\right)_{*} \nu_{n}\right)\left(V_{s}\right),
$$

(here the $V_{s}$ correspond to the partition $Q$ and to the function $\eta$ ).

That term is less than

$$
-\frac{1}{q(n-2 q+1)} \sum_{j=0}^{q-1} \sum_{t \in S_{2}(j)} \int_{\left\{\eta \leq e^{-s_{0}}\right\}} \log \eta d\left(f^{t}\right)_{*} \nu_{n} .
$$

Finally:

$$
\sum_{t \in S_{2}(j)}\left(f^{t}\right)_{*} \nu_{n} \leq n \mu_{n}
$$

and since $\int_{\left\{\eta \leq e^{-s_{0}}\right\}} \log \eta d \mu_{n}$ converges to $\int_{\left\{\eta \leq e^{\left.-s_{0}\right\}}\right.} \log \eta d \mu$, it is also as small as we want if $s_{0}$ is large enough then $n$ is large enough. This gives the third point of Proposition 2.3.8, and the proposition follows. 


\section{CHAPTER 3}

\section{DYNAMICS OF BIRATIONAL MAPS OF $\mathbb{P}^{k}$}

\subsection{A family of birational maps}

The purpose of this section is to introduce the family of birational maps $\mathscr{B}$.

Recall that a meromorphic map $f: \mathbb{P}^{k} \rightarrow \mathbb{P}^{k}$ is holomorphic outside an analytic subset $I(f)$ of codimension $\geq 2$ in $\mathbb{P}^{k}$. It can be written in homogeneous coordinates as $\left[P_{0}: \ldots: P_{k}\right]$ where the $P_{i}$ are homogeneous polynomials of algebraic degree $d$ in the $\left(z_{0}, \ldots, z_{k}\right)$ variable, with $\operatorname{gcd}_{i}\left(P_{i}\right)=1$.

Let $\Gamma$ denote the closure of the graph of the restriction of $f$ to $\mathbb{P}^{k} \backslash I(f)$. This is an irreducible analytic set of dimension $k$ in $\mathbb{P}^{k} \times \mathbb{P}^{k}$.

Let $\pi_{1}$ and $\pi_{2}$ denote the canonical projections of $\mathbb{P}^{k} \times \mathbb{P}^{k}$ on its factors. The indeterminacy locus $I(f)$ (or indeterminacy set) of $f$ is the set of points $z \in \mathbb{P}^{k}$ such that $\operatorname{dim} \pi_{1}^{-1}(z) \cap \Gamma \geq 1$. It is also the common zeros of the $P_{i}: I(f)=\left\{P_{i}=0\right\}$.

We assume that $f$ is dominant, that is, $\pi_{2}(\Gamma)=\mathbb{P}^{k}$. If $A$ is a subset of $\mathbb{P}^{k}$, define

$$
f(A):=\pi_{2}\left(\pi_{1}^{-1}(A) \cap \Gamma\right) \quad \text { and } \quad f^{-1}(A):=\pi_{1}\left(\pi_{2}^{-1}(A) \cap \Gamma\right) .
$$

Define formally for a current $S$ on $\mathbb{P}^{k}$, not necessarily positive or closed, the pull-back $f^{*}(S)$ by

$$
f^{*}(S):=\left(\pi_{1}\right)_{*}\left(\pi_{2}^{*}(S) \wedge[\Gamma]\right)
$$

where $[\Gamma]$ is the current of integration on $\Gamma$.

Similarly, the operator $f_{*}$ is formally defined by

$$
f_{*}(R):=\left(\pi_{2}\right)_{*}\left(\pi_{1}^{*}(R) \wedge[\Gamma]\right) .
$$

Here, we consider a birational map $f$ of algebraic degree $d \geq 2$. That is a map such that $\# f^{-1}(z)=1$ for $z$ generic. Let $\delta$ be the algebraic degree of $f^{-1}$. We denote $I^{+}:=I(f)$ and $I^{-}=I\left(f^{-1}\right)$ the indeterminacy sets of $f$ and $f^{-1}$. 
We also consider the critical sets $\mathscr{C}^{+}$(or $\left.\mathscr{C}(f)\right)$ and $\mathscr{C}^{-}\left(\right.$or $\left.\mathscr{C}\left(f^{-1}\right)\right)$ defined by:

$$
\begin{aligned}
& \mathscr{C}^{+}:=f^{-1}\left(I^{-}\right) \\
& \mathscr{C}^{-}:=\left(f^{-1}\right)^{-1}\left(I^{+}\right) .
\end{aligned}
$$

Write $f=\left[P_{0}: \ldots: P_{k}\right]$ where the $P_{i}$ are homogeneous polynomials of degree $d$. Let $F=\left(P_{0}, \ldots, P_{k}\right)$ be the induced map on $\mathbb{C}^{k+1}$. Similarly, write $f^{-1}=\left[Q_{0}: \ldots: Q_{k}\right]$ where the $Q_{i}$ are homogeneous polynomials of degree $\delta$ and let $F^{-1}=\left(Q_{0}, \ldots, Q_{k}\right)$. There is of course an abuse of notation since $F \circ F^{-1} \neq I d$. Instead, we have that:

$$
F \circ F^{-1}=P\left(z_{0}, \ldots, z_{k}\right) \times\left(z_{0}, \ldots, z_{k}\right),
$$

where $P$ is a homogeneous polynomial of degree $d \delta-1$ equal to 0 in $\pi^{-1}\left(\mathscr{C}^{-} \cup I^{-}\right)$ where $\pi: \mathbb{C}^{k+1} \rightarrow \mathbb{P}^{k}$ is the canonical projection. That implies that the critical set $\mathscr{C}^{-}$is an analytic set of codimension 1 and that $I^{-} \subset \mathscr{C}^{-}$. Similarly, we have that $\mathscr{C}^{+}$is of codimension 1 and $I^{+} \subset \mathscr{C}^{+}$(see also Proposition 3.3 in [15] and [50]). So, $f: \mathbb{P}^{k} \backslash \mathscr{C}^{+} \rightarrow \mathbb{P}^{k} \backslash \mathscr{C}^{-}$is a biholomorphism.

For $0 \leq q \leq k$ and $n>0$, define $\lambda_{q}\left(f^{n}\right)$ as the degree of $\left(f^{n}\right)^{-1}(L)$ for $L$ a generic linear subspace of codimension $q$. The number $\lambda_{q}\left(f^{n}\right)$ gives a size for the action of $f^{n}$ on the cohomology group $H^{q, q}\left(\mathbb{P}^{k}\right)$. Since the quantity $\lambda_{q}\left(f^{n}\right)$ is cohomological, we have:

$$
\begin{aligned}
\lambda_{q}\left(f^{n}\right) & =\left\|\left(f^{n}\right)^{*}\left(\omega^{q}\right)\right\|=\int_{\mathbb{P}^{k}}\left(f^{n}\right)^{*}\left(\omega^{q}\right) \wedge \omega^{k-q} \\
& =\left\|\left(f^{n}\right)_{*}\left(\omega^{k-q}\right)\right\|=\int_{\mathbb{P}^{k}}\left(f^{n}\right)_{*}\left(\omega^{k-q}\right) \wedge \omega^{q} .
\end{aligned}
$$

We have in particular that $\lambda_{1}(f)=d$ is the algebraic degree and that $\lambda_{k}\left(f^{n}\right)$ the topological degree of $f^{n}$ is equal to $\#\left(f^{n}\right)^{-1}(z)$ for $z$ generic. In particular, $\lambda_{k}\left(f^{n}\right)=1$ since $f$ is birational.

We define the dynamical degree of order $q$ of $f$ by:

$$
d_{q}:=\lim _{n \rightarrow \infty}\left(\lambda_{q}\left(f^{n}\right)\right)^{\frac{1}{n}}
$$

These limits always exist and $d_{q} \leq d_{1}^{q}$ [25]. The last degree $\lambda_{k}(f)=d_{k}$ is equal to 1 .

A result by Gromov [40, Theorem 1.6] implies that $q \mapsto \log d_{q}$ is concave in $q$. In particular, there exists a $q_{0}$ such that:

$$
1=d_{0} \leq d_{1} \leq \cdots \leq d_{q_{0}} \geq \cdots \geq d_{k}=1 .
$$

Denote for $f^{-1}, \lambda_{q}^{-}\left(f^{n}\right)$ and $d_{q}^{-}$the quantities previously defined for $f$.

In order to work with the currents in cohomology, we need a hypothesis on the indeterminacy sets so that $\left(f^{n}\right)^{*}=\left(f^{*}\right)^{n}$ on the cohomology group $H^{q, q}\left(\mathbb{P}^{k}\right)$. If so, we say that the map is algebraically q-stable (see [50] and [31]). For that purpose, we will consider the following class of mappings. 
Definition 3.1.1. - Let $\mathscr{B}_{0}$ be the set of birational maps of $\mathbb{P}^{k}$ such that there exist an integer $0<s<k$ and two integers $d, \delta>1$ such that $d^{s}=\delta^{k-s}$ and for all $n \geq 1$ :

- for all linear subspaces of codimension $q \leq s, \operatorname{deg}\left(f^{-n} L\right)=d^{q n}$;

- for all linear subspaces of codimension $q \leq k-s, \operatorname{deg}\left(f^{n} L\right)=\delta^{q n}$.

Of course, the fact that $d^{s}=\delta^{k-s}$ can be deduced from the rest of the definition but we keep it there for simplicity. If a map $f$ is in $\mathcal{B}_{0}$, then $f$ is algebraically $q$-stable for $q \leq s$ and $f^{-1}$ is algebraically $q$-stable for $q \leq k-s$. The following classical lemmas will help us in producing a more geometric characterisation of $\mathscr{B}_{0}$.

LEMMA 3.1.2. - Let $f$ be a birational map of algebraic degree $d$ such that $\operatorname{dim}\left(I^{+}\right)=$ $k-s-1$ then $\lambda_{q}(f)=d^{q}$ for all $q \leq s$ and $\lambda_{s+1}(f)<d^{s+1}$.

Proof. - Let $f$ be as above and let $q \leq s$. Then $\lambda_{q}(f)$ is the mass of the current $f^{*}\left(\omega^{q}\right)$ which is a form with $L^{1}$ coefficients. In particular, $f^{*}\left(\omega^{q}\right)$ gives no mass to $I^{+}$. On the other hand $f^{*}\left(\omega^{q}\right)=f^{*}(\omega)^{q}$ outside $I^{+}$. But the current $f^{*}(\omega)^{q}$ is a well defined positive closed current of bidimension $(k-q, k-q)$ of mass $d^{q}$ (see Corollary 4.11 in [14, Chapter III]), and it cannot charge $I^{+}$since $k-s-1<k-q$. So we have the equality $f^{*}\left(\omega^{q}\right)=f^{*}(\omega)^{q}$ on $\mathbb{P}^{k}$ and both currents have the same mass $d^{q}$.

Now the same argument will give that $\lambda_{s+1}(f)<d^{s+1}$ if $f^{*}(\omega)^{s+1}$ gives some mass to $I^{+}$(Corollary 4.11 in [14, Chapter III] still implies that $f^{*}(\omega)^{s+1}$ is well defined). Let $u$ be a potential of $f^{*}(\omega)$ that is $d d^{c} u+d \omega=f^{*}(\omega)$. We will see in Lemma 3.2.4 below that:

$$
u(x) \leq A^{\prime} \log \operatorname{dist}\left(x, I^{+}\right)+B^{\prime} .
$$

In particular, since $\log \operatorname{dist}\left(x, I^{+}\right) \leq \log \operatorname{dist}(x, y)$ for $y \in I^{+}$, Theorem 7.8 in [14, Chapter III] shows the current $f^{*}(\omega)^{s+1}$ has its Lelong numbers $>c>0$ on $I^{+}$for some $c$. Siu's theorem [51] implies that this current is greater than $c\left[I^{+}\right]$. In particular, it gives some mass to $\left[I^{+}\right]$.

Applying the previous lemma to a map $f \in \mathcal{B}_{0}$ shows that necessarily $\operatorname{dim}\left(I^{+}\right)=$ $k-s-1$ and $\operatorname{dim}\left(I^{-}\right)=s-1$. We now prove:

LEMMA 3.1.3. - If a map $f$ is in $\mathscr{B}_{0}$ with $s$ as above, then for any $q \leq s$, no algebraic set of codimension $q$ is sent to $I^{+}$by some iterate $f^{n}$ of $f$.

Proof. - Assume that there exist $f \in \mathscr{B}_{0}$ and an algebraic set $A$ of codimension $q \leq s$ sent to $I^{+}$by $f^{n}$. To simplify the proof, assume that $n=1$ and take the smallest $q$ such that the above property is satisfied. Let $f=\left[P_{0}: P_{1} \ldots: P_{k}\right]$ where the $P_{i}$ are homogeneous polynomials of algebraic degree $d$ with no common factors. That way, $I^{+}=\left\{P_{0}=\cdots=P_{k}=0\right\}$. Let $\pi: \mathbb{C}^{k+1} \rightarrow \mathbb{P}^{k}$ be the canonical projection and let $F=\left(P_{0}, \ldots, P_{k}\right)$. 
For an element $Z=\left(z_{0}, \ldots, z_{k}\right) \in \mathbb{C}^{k+1}$, we write $|Z|^{2}=\left|z_{0}\right|^{2}+\cdots+\left|z_{k}\right|^{2}$. Then, we have that:

$$
f^{*}(\omega)-d \omega=d d^{c}\left(\log |F|^{2}-d \log |Z|^{2}\right) .
$$

Applying $f^{*}$ again gives:

$$
f^{*}\left(f^{*}(\omega)\right)-d f^{*}(\omega)=d d^{c}\left(\log \left|F^{2}\right|^{2}-d \log |F|^{2}\right) .
$$

So, taking the sum gives:

$$
f^{*}\left(f^{*}(\omega)\right)-d^{2} \omega=d d^{c}\left(\log \left|F^{2}\right|^{2}-d^{2} \log |Z|^{2}\right) .
$$

The singularities of the qpsh function $\varphi:=\log \left|F^{2}\right|^{2}-d^{2} \log |Z|^{2}$ are exactly on the set $A \cup I^{+}$(assuming $A$ is the largest algebraic set sent to $I^{+}$) so we have the inequality $\varphi \leq C \log \operatorname{dist}\left(x, A \cup I^{+}\right)+C$ for some $C>0$.

Again, Corollary 4.11 in [14, Chapter III] implies that $f^{*}\left(f^{*}(\omega)\right)^{q}$ is well defined and Theorem 7.8 in [14, Chapter III] shows that the current $\left(f^{*}\left(f^{*}(\omega)\right)\right)^{q}$ has its Lelong numbers $>c>0$ on $A$ for some $c$. Siu's theorem [51] implies that it gives positive mass to $A$. But $f^{*}\left(f^{*}(\omega)\right)^{q}$ is of mass $d^{2 q}$ and $f^{*}\left(f^{*}(\omega)\right)^{q}=\left(f^{2}\right)^{*}(\omega)^{q}$ outside $A \cup I^{+}$. Since the current $\left(f^{2}\right)^{*}\left(\omega^{q}\right)$ gives no mass to analytic set we have $\lambda_{q}\left(f^{2}\right)<$ $\lambda_{q}(f)^{2}$, a contradiction.

This leads to the lemma:

LEMMA 3.1.4. - Let $f$ be a birational map with $\operatorname{dim}\left(I^{+}\right)=k-s-1$ and $\operatorname{dim}\left(I^{-}\right)=$ $s-1$. Then the fact that no algebraic set of codimension $q \leq s$ is sent to $I^{+}$by some iterate $f^{n}$ of $f$ is equivalent to the condition:

$$
\bigcup_{n \geq 0} f^{-n} I(f) \cap \bigcup_{n \geq 0} f^{n} I\left(f^{-1}\right)=\varnothing .
$$

Proof. - Assume that there exists some $A$ sent to $I^{+}$by $f^{n}$. Then we have that $A \subset$ $\mathscr{C}\left(f^{n}\right)$ since $A$ is contracted and so $f^{n}(A) \subset I\left(f^{-n}\right)$. Since $I\left(f^{-n}\right) \subset \bigcup_{0}^{n-1} f^{j} I\left(f^{-1}\right)$ we have that the intersection (7) is not empty.

Now assume that the intersection (7) is not empty. Formal manipulations show that (7) is equivalent to:

$$
I^{-} \cap \bigcup_{n \geq 0} f^{-n} I(f)=\varnothing .
$$

Hence we can assume that there exists some point $y \in I^{-} \cap f^{-n} I(f)$. The map $f: \mathscr{C}^{+} \rightarrow I^{-}$is meromorphic and surjective, in particular, the $\operatorname{dimension} \operatorname{dim}\left(f^{-1}(x)\right)$ is upper semi continuous hence $\geq k-s$ at every point. In particular, the set $f^{-1}(y)$ is of codimension $\leq s$ and it is sent by $f^{n+1}$ to $I(f)$. This concludes the proof.

This leads to the characterisation of $\mathcal{B}_{0}$ : 
Proposition 3.1.5. - Let $f$ be a birational map with $\operatorname{dim}\left(I^{+}\right)=k-s-1$ and $\operatorname{dim}\left(I^{-}\right)=s-1$, then $f \in \mathscr{B}_{0}$ if and only if $f$ satisfies (7).

Proof. - The previous lemmas already give the first implication.

Take $f$ a birational map with $\operatorname{dim}\left(I^{+}\right)=k-s-1$ and $\operatorname{dim}\left(I^{-}\right)=s-1$ which satisfies (7). Lemma 3.1.2 gives that $\lambda_{q}(f)=d^{q}$ for $q \leq s$ and $\lambda_{q}\left(f^{-1}\right)=\delta^{q}$ for $q \leq k-s$ where $d$ is the algebraic degree of $f$ and $\delta$ is the algebraic degree of $f^{-1}$. The proof follows from the following lemma.

We introduce some notations first. Let $\mathscr{C}_{s}$ denote the convex compact set of (strongly) positive closed currents $S$ of bidegree $(s, s)$ on $\mathbb{P}^{k}$ and of mass 1, i.e. $\|S\|:=\left\langle S, \omega^{k-s}\right\rangle=1$. For a positive closed current $T$ of mass $m(T)>0$, we denote by $T_{\text {nor }}$ the renormalization of $T$ (that is $T_{\text {nor }}=m(T)^{-1} T$ ). Denote for simplicity $L:=\lambda_{q}(f)^{-1} f^{*}$ and $\Lambda:=\left(\lambda_{k-q}\right)^{-1} f_{*}=\left(\lambda_{q}^{-}(f)\right)^{-1} f_{*}$ which are well defined operators on the elements of $\mathscr{C}_{q}$ which are smooth near $I^{-}$(resp. $I^{+}$). We make an abuse of notations and write $L$ instead of $L_{q}$, this is not a problem since in what follows $L(S)$ will always be the current $f^{*}(S)_{\text {nor }}$. The theory of super-potentials (see the appendix) allows us to extend the operator $L$ (resp. $\Lambda$ ) to the currents in $\mathscr{C}_{q}$ such that their super-potentials are finite at one point of the form $\Lambda(S)$ for $S \in \mathscr{C}_{k-q+1}$ smooth near $I^{+}$(resp. at one point of the form $L(S)$ for $S \in \mathscr{C}_{k-q+1}$ smooth near $I^{-}$).

Now we show that a map which satisfies (7) is in fact algebraically $q$-stable for all $q$. That is to say no mass is lost on the indeterminacy set by pull-back. More precisely, we have the proposition that uses the theory of super-potential:

LEMмa 3.1.6. - Let $f$ be a birational map satisfying (7). Then for all $0 \leq q \leq k$, $\lambda_{q}\left(f^{n}\right)=\left(\lambda_{q}(f)\right)^{n}$ for all $n$ and so $d_{q}=\lambda_{q}(f)$ for all $q$.

Proof. - We have to compute the integral:

$$
\lambda_{q}\left(f^{n}\right)=\left\|\left(f^{n}\right)^{*}\left(\omega^{q}\right)\right\|=\int_{\mathbb{P}^{k}}\left(f^{n}\right)^{*}\left(\omega^{q}\right) \wedge \omega^{k-q} .
$$

The proof is by induction on $n$ : $\left(f^{n-1}\right)^{*}\left(\omega^{q}\right)$ is a form in $L^{1} \operatorname{smooth}$ near $I^{-}$by (7). So we can define its pull-back by $f$ which is of mass $\lambda_{q}(f) \lambda_{q}\left(f^{n-1}\right)$. On the other hand, $\omega^{q}$ is smooth near $I\left(f^{-n}\right)$ so it is $\left(f^{n}\right)^{*}$-admissible and the mass of $\left(f^{n}\right)^{*}\left(\omega^{q}\right)$ is $\lambda_{q}\left(f^{n}\right)$.

We will now prove that $f^{*}\left(\left(f^{n-1}\right)^{*}\left(\omega^{q}\right)\right)=\left(f^{n}\right)^{*}\left(\omega^{q}\right)$.

Let $\pi_{1 \mid \Gamma}$ and $\pi_{2 \mid \Gamma}$ be the restriction of $\pi_{1}$ and $\pi_{2}$ to the graph $\Gamma$ of $f$. That way, $f^{*}(S)=\left(\pi_{1}\right)_{*}\left(\pi_{2 \mid \Gamma}\right)^{*}(S)$ where $S \in \mathscr{C}_{q}$ is smooth near $I^{-}$. We will take $S=\left(f^{n-1}\right)^{*}\left(\omega^{q}\right)_{\text {nor }}$.

Let $V$ be a small neighborhood of $I^{-}$such that $S$ is smooth here. Outside $\pi_{2}{ }^{-1}(V) \cap$ $\Gamma, \pi_{2 \mid \Gamma}$ is a finite map, hence $\pi_{2}{ }^{*}(S) \wedge[\Gamma]$ is well defined and depends continuously of $S$ here by Theorem 1.1 in [29]. Furthermore, if $S_{\mid \mathbb{P}^{k} \backslash V}$ does not give mass to a Borel 
set $A$ then $\left(\pi_{2 \mid \Gamma}\right)^{*}(S)$ does not give mass to $\left(\pi_{2 \mid \Gamma}\right)^{-1}(A)$ outside $\pi_{2}^{-1}(V) \cap \Gamma$. Since $\pi_{1}$ is holomorphic, the same is true for $f^{*}\left(S_{\mid \mathbb{P} k} \backslash V\right)$. On $V, S$ is smooth, hence $f^{*}\left(S_{\mid V}\right)$ is a form in $L^{1}$ (see e.g. [25]).

We consider $S=\left(f^{n-1}\right)^{*}\left(\omega^{q}\right):\left(f^{n-1}\right)^{*}\left(\omega^{q}\right)$ is a form in $L^{1}$ hence it does not give mass to algebraic sets of dimension $\leq k-1$; so $f^{*}\left(\left(f^{n-1}\right)^{*}\left(\omega^{q}\right)\right)$ is a current that does not give mass to algebraic sets of dimension $\leq k-1$. We get that $f^{*}\left(\left(f^{n-1}\right)^{*}\left(\omega^{q}\right)\right)$ and $\left(f^{n}\right)^{*}\left(\omega^{q}\right)$ are equal wherever they are smooth that is outside analytic sets of dimension $\leq k-1$. We deduce that they are equal hence they have the same mass.

The following corollary of the previous proof will be used later:

Corollary 3.1.7. - Let $R \in \mathscr{C}_{q}$ be a smooth form and $f$ in $\mathscr{B}_{0}$. Then for all $i, j \geq 0$, we have that $\left(f^{i}\right)^{*}(R)$ is $\left(f^{j}\right)^{*}$-admissible and $\left(f^{j}\right)^{*}\left(\left(f^{i}\right)^{*}(R)\right)=\left(f^{i+j}\right)^{*}(R)$.

Let $j \geq 0$ and $q \leq k$. Let $f$ in $\mathscr{B}_{0}$. For a current $S \in \mathscr{C}_{q}$ which is $\left(f^{j}\right)^{*}$-admissible, we can define $L_{j}(S)$ as $\left(\lambda_{q}\left(f^{j}\right)\right)^{-1}\left(f^{j}\right)^{*}(S)$ (similarly we define $\left.\Lambda_{j}\right)$. By Proposition 3.1.6, we have that $\lambda_{q}\left(f^{j}\right)=\lambda_{q}(f)^{j}$ so we can also write $L_{j}(S)=\lambda_{q}(f)^{-j}\left(f^{j}\right)^{*}(S)$. From Corollary 3.1.7, we have that $L^{j}(S)=L_{j}(S)$ on smooth forms, the question is: does it also stand for $\left(f^{j}\right)^{*}$-admissible currents ? The following lemma answers positively:

Lemma 3.1.8. - Let $S \in \mathscr{C}_{q}$ for $q \leq k$. Let $n>0$ such that $S$ is $\left(f^{n}\right)^{*}$-admissible then for all $j$ with $0 \leq j \leq n-1, L^{j}(S)$ is well defined, $f^{*}$-admissible and $L^{j+1}(S)=$ $L_{j+1}(S)$. In particular, $L^{n}(S)=L_{n}(S)$.

Proof. - Let $S$ be as above, then a super-potential of $S$ is finite at $\Lambda_{n}\left(\omega^{k-q+1}\right)$ by hypothesis. Since $f$ satisfies $(7)$, we have that $\Lambda\left(\omega^{k-q+1}\right)$ is smooth near $I\left(f^{n-1}\right)$, the previous corollary implies that

$$
\Lambda_{n}\left(\omega^{k-q+1}\right)=\Lambda_{n-1}\left(\Lambda\left(\omega^{k-q+1}\right)\right) .
$$

So the super-potentials of $S$ are finite at the image by $\Lambda_{n-1}$ of a current smooth near $I\left(f^{n-1}\right)$ : it is $\left(f^{n-1}\right)^{*}$-admissible (see the appendix). An immediate induction gives that $S$ is $\left(f^{j}\right)^{*}$-admissible for $j \leq n$.

Now we prove by induction on $j$ that $L^{j}(S)$ is $f^{*}$-admissible and that $L^{j+1}(S)=$ $L_{j+1}(S)$. In order to do that, we show that both currents have the same superpotential and conclude by Proposition A.1.3.

For $j=0$, it is just the fact that $S$ is $f^{*}$-admissible. Now, assume the property holds up to the rank $j$. A super-potential of $L^{j}(S)=L_{j}(S)$ is by Proposition A.1.18:

$$
u_{L_{j}(S)}=u_{L_{j}\left(\omega^{q}\right)}+\frac{\lambda_{q-1}\left(f^{j}\right)}{\lambda_{q}\left(f^{j}\right)} u_{S} \circ \Lambda_{j}
$$


on forms smooth near $I\left(f^{j}\right)$. Taking the value at $\Lambda\left(\omega^{k-q+1}\right)$ (which is smooth near $\left.I\left(f^{j}\right)\right)$ gives:

$$
U_{L_{j}(S)}\left(\Lambda\left(\omega^{k-q+1}\right)\right)=\mathcal{U}_{L_{j}\left(\omega^{q}\right)}\left(\Lambda\left(\omega^{k-q+1}\right)\right)+\frac{\lambda_{q-1}\left(f^{j}\right)}{\lambda_{q}\left(f^{j}\right)} u_{S}\left(\Lambda_{j}\left(\Lambda\left(\omega^{k-q+1}\right)\right)\right) .
$$

The current $L_{j}\left(\omega^{q}\right)=L^{j}\left(\omega^{q}\right)$ is $f^{*}$-admissible since it is smooth near $I^{-}$, that implies that $U_{L_{j}\left(\omega^{q}\right)}\left(\Lambda\left(\omega^{k-q+1}\right)\right)$ is finite. Similarly, applying the previous corollary to $f^{-1}$ gives that $\Lambda_{j}\left(\Lambda\left(\omega^{k-q+1}\right)\right)=\Lambda^{j+1}\left(\omega^{k-q+1}\right)$ and since $S$ is $\left(f^{j+1}\right)^{*}$-admissible then $U_{S}\left(\Lambda_{j}\left(\Lambda\left(\omega^{k-q+1}\right)\right)\right)$ is also finite.

That gives that $U_{L_{j}(S)}\left(\Lambda\left(\omega^{k-q+1}\right)\right)$ is finite so $L_{j}(S)$ is $f^{*}$-admissible. We can now apply Proposition A.1.18 to $L^{j}(S)$ :

$$
\begin{aligned}
\mathcal{U}_{L^{j+1}(S)} & =\mathcal{U}_{L\left(\omega^{q}\right)}+\frac{\lambda_{q-1}(f)}{\lambda_{q}(f)} \mathcal{U}_{L^{j}(S)} \circ \Lambda \\
& =\mathcal{U}_{L\left(\omega^{q}\right)}+\frac{\lambda_{q-1}(f)}{\lambda_{q}(f)}\left(\mathcal{U}_{L_{j}\left(\omega^{q}\right)}+\frac{\lambda_{q-1}\left(f^{j}\right)}{\lambda_{q}\left(f^{j}\right)} \mathcal{U}_{S} \circ \Lambda_{j}\right) \circ \Lambda
\end{aligned}
$$

on smooth forms. Since $U_{L\left(\omega^{q}\right)}+\frac{\lambda_{q-1}(f)}{\lambda_{q}(f)} U_{L_{j}\left(\omega^{q}\right)} \circ \Lambda=\mathcal{U}_{L^{j+1}\left(\omega^{q}\right)}$ on smooth forms, and since $\Lambda_{j} \circ \Lambda=\Lambda_{j+1}$ on smooth forms, we deduce from Proposition 3.1.6 that:

$$
U_{L^{j+1}(S)}=U_{L_{j+1}(S)}
$$

on smooth forms, hence $L^{j+1}(S)=L_{j+1}(S)$ by Proposition A.1.3. That gives the lemma.

Summing up what we proved, we have that for an element $f \in \mathcal{B}_{0}$ the dynamical degrees are ordered as follows:

$$
\begin{aligned}
& d_{0}<d_{1}<d_{2}<\ldots<d_{s} \quad>\ldots>d_{k-2}>d_{k-1}>d_{k} \\
& \begin{array}{llllll}
\| 11 & \| & \| & \|
\end{array} \\
& 1<d<d^{2}<\ldots<d^{s}=\delta^{k-s}>\ldots>\quad \delta^{2}>\quad \delta>\quad 1 .
\end{aligned}
$$

Let $f \in \mathscr{B}_{0}$. Recall that a quasi-potential of a current $T \in \mathscr{C}_{q}$ is a current $U$ of bidegree $(q-1, q-1)$ such that $T=\omega^{q}+d d^{c} U$. We know from the appendix that it is always possible to take $U$ negative. In what follows, for an irreducible analytic set $A$, we define $[f(A)]$ as the current of integration over $f(A)$ counting the multiplicity of $f$ at $A$ and if $A$ is not irreducible, we decompose it into irreducible components $\left(A_{i}\right)$ and we define $[f(A)]$ as $\sum_{i}\left[f\left(A_{i}\right)\right]$.

Since $f$ satisfies (7), we have that $I^{+} \cap f^{j}\left(I^{-}\right)=\varnothing$ for $j \leq n$. So, $f^{n}\left(I^{-}\right)$is well defined and the form $U_{L(\omega)} L\left(\omega^{s-1}\right)$ is smooth in $f^{n}\left(I^{-}\right)$so the following integral is well defined:

$$
\int_{\left[f^{n}\left(I^{-}\right)\right]} U_{L(\omega)} L\left(\omega^{s-1}\right)
$$


The terms $\left(\operatorname{vol}\left(I^{-}\right)\right)^{-1}$ and $\left(\operatorname{vol}\left(I^{+}\right)\right)^{-1}$ in the following hypothesis are just here to normalize the integrals. We are going to restrict the study to the subset $\mathscr{B}$ of $\mathscr{B}_{0}$ given by the following definition:

DEFINITION 3.1.9. - Let $U_{L(\omega)}$ be a negative quasi-potential of $L(\omega)$ and let $U_{\Lambda(\omega)}$ be a negative quasi-potential of $\Lambda(\omega)$. The set $\mathscr{B}$ is the set of elements $f \in \mathscr{B}_{0}$ such that:

$$
\begin{aligned}
& \sum_{n=0}^{\infty}\left(\frac{1}{d^{s}}\right)^{n}\left(\operatorname{vol}\left(I^{-}\right)\right)^{-1} \int_{f^{n}\left(I^{-}\right)} U_{L(\omega)} L\left(\omega^{s-1}\right)>-\infty \\
& \text { and } \\
& \sum_{n=0}^{\infty}\left(\frac{1}{\delta^{k-s}}\right)^{n}\left(\operatorname{vol}\left(I^{+}\right)\right)^{-1} \int_{f^{-n}\left(I^{+}\right)} U_{\Lambda(\omega)} \Lambda\left(\omega^{k-s-1}\right)>-\infty .
\end{aligned}
$$

As usual, for two sets $E$ and $F$, we denote $\inf _{x \in E, y \in F} \operatorname{dist}(x, y)$ by $\operatorname{dist}(E, F)$. In [1], the authors asked for a quantitative and stronger version of (7) similar to:

Hypothesis 3.1.10. - The birational mapping $f$ satisfies $\operatorname{dim}\left(I^{+}\right)=k-s-1$, $\operatorname{dim}\left(I^{-}\right)=s-1$ and:

$$
\begin{aligned}
& \sum_{n=0}^{\infty}\left(\frac{1}{d}\right)^{n} \log \operatorname{dist}\left(I^{+}, f^{n}\left(I^{-}\right)\right)>-\infty \\
& \quad \text { and } \\
& \sum_{n=0}^{\infty}\left(\frac{1}{\delta}\right)^{n} \log \operatorname{dist}\left(I^{-}, f^{-n}\left(I^{+}\right)\right)>-\infty .
\end{aligned}
$$

In $\mathbb{P}^{2}$, the convergence of the series $\sum_{n=0}^{\infty} d^{-n} \log \operatorname{dist}\left(I^{+}, f^{n}\left(I^{-}\right)\right)>-\infty$ and $\sum_{n=0}^{\infty} \delta^{-n} \log \operatorname{dist}\left(I^{-}, f^{-n}\left(I^{+}\right)\right)>-\infty$ are equivalent (see [15]), it has no reason to be true in higher dimension.

In the case of $\mathbb{P}^{2}$, a map is in $\mathcal{B}$ if and only if it satisfies Hypothesis 3.1 .10 (see [1, Theorem 4.3] and Theorem 3.2.1 below). That is because the distance between the supports of the currents is a good distance for Dirac masses but not for currents of higher bidimension. We will see in Theorem 3.2.8 that Hypothesis 3.1.10 implies that a map is in $\mathcal{B}$.

We will see in Theorem 3.2.1 that the convergence of the series in Definition 3.1.9 has a clear interpretation in term of super-potentials : it means exactly that the super-potentials of the Green current of order $s$ are finite at $\left[I^{-}\right]_{\text {nor }}$.

Generalizing [1] to the case of higher dimension is the main goal of this chapter. The class $\mathscr{B}$ contains the regular automorphisms of $\mathbb{C}^{k}$ since those satisfy $f^{n}\left(I^{-}\right)=I^{-}$, $f^{-n}\left(I^{+}\right)=I^{+}$and $I^{+} \cap I^{-}=\varnothing$ (see [50]). It also contains the regular birational maps of $\mathbb{P}^{k}([\mathbf{2 3}])$, in this case the indeterminacy sets of $I^{+}$and $I^{-}$are trapped in 
some stable disjoint open sets. The interest of $\mathcal{B}$ is that it is generic in the following sense:

TheOREm 3.1.11. - Let $E_{s}$ be the set of birational maps $f: \mathbb{P}^{k} \rightarrow \mathbb{P}^{k}$ such that $I^{+}$ and $I^{-}$satisfy $\operatorname{dim}\left(I^{+}\right)=k-s-1$ and $\operatorname{dim}\left(I^{-}\right)=s-1$. Consider the group action:

$$
\begin{aligned}
\Phi: \operatorname{PGL}(k+1, \mathbb{C}) \times E_{s} & \rightarrow E_{s} \\
(A, f) & \mapsto A \circ f .
\end{aligned}
$$

Then outside a pluripolar set of the orbit $\operatorname{Orb}(f)$ of $f \in E_{s}$, the maps of Orb $(f)$ are in $\mathscr{B}$.

This provides us with many new examples: start with a regular polynomial automorphism of $\mathbb{C}^{k}$ or a regular birational map in $\mathbb{P}^{k}$ then consider its orbit under the action of $\mathrm{PGL}(k+1, \mathbb{C})$. Section 3.3 is devoted to the proof of the previous theorem.

Other families of polynomial automorphisms were studied in [44] and [42]. In these papers, the authors replace the set $I^{-}$by the set $f\left(L_{\infty} \backslash I^{+}\right)$in the condition $I^{+} \cap I^{-}=$ $\varnothing$. Those mappings are not always in $\mathcal{B}$ since the conditions $\operatorname{dim}\left(I^{+}\right)=k-s-1$ and $\operatorname{dim}\left(I^{-}\right)=s-1$ no longer need to be satisfied.

\subsection{Construction of the Green currents}

Take $f \in \mathscr{B}$. Let $s$ be such that $\operatorname{dim}\left(I^{+}\right)=k-s-1$ and $\operatorname{dim}\left(I^{-}\right)=s-1$. The following theorem is the main result of this section.

TheOREM 3.2.1. - Let $f \in \mathcal{B}$, then the sequence $\left(L^{m}\left(\omega^{s}\right)\right)$ converges in the Hartogs' sense to the Green current of order $s$ of $f$ that we denote by $T_{s}^{+}$.

To prove the theorem, we need the following computation of the super-potential of $L^{m}\left(\omega^{q}\right)$ for $q \leq s$,

LEMma 3.2.2. - A super-potential of $L^{m}\left(\omega^{q}\right)$ is given on $R \in \mathscr{C}_{k-q+1}$ which is $\left(f^{m}\right)_{*}$-admissible by:

$$
\sum_{n=0}^{m-1}\left(\frac{1}{d}\right)^{n} \mathcal{u}_{L\left(\omega^{q}\right)} \circ \Lambda^{n}(R) .
$$

Proof. - Recall that for $q \leq s$, we have $\lambda_{q}\left(f^{n}\right)=\left(d^{q}\right)^{n}$ for all $n$ and so $d_{q}=d^{q}$. Recall that for $S \in \mathscr{C}_{q}$ which is $f^{*}$-admissible, $L(S)$ is the element of $\mathscr{C}_{q}$ defined as $d^{-q} f^{*}(S)$. Furthermore, any current smooth in a neighborhood of $I^{-}$is $f^{*}$-admissible. By Corollary 3.1.7, $L^{n-1}\left(\omega^{q}\right)$ is $f^{*}$-admissible since $f$ satisfies (7) so we can define $L^{n}\left(\omega^{q}\right)$.

Now, let $U_{L\left(\omega^{q}\right)}$ denote a negative super-potential of $L\left(\omega^{q}\right)$ (it is always possible by Proposition A.1.1). Assume first that $R \in \mathscr{C}_{k-q+1}$ is smooth. So, we have that 
by Proposition A.1.18 that a super-potential of $L^{m}\left(\omega^{q}\right)=L\left(L^{m-1}\left(\omega^{q}\right)\right)$ is given on currents in $\mathscr{C}_{k-q+1}$ smooth in a neighborhood of $I^{+}$by:

$$
u_{L\left(\omega^{q}\right)}+\frac{1}{d} u_{L^{m-1}\left(\omega^{q}\right)} \circ \Lambda .
$$

So, by induction, for a smooth element $R \in \mathscr{C}_{k-q+1}$, we have that a super-potential of $L^{m}\left(\omega^{q}\right)$ is given on $R$ by:

$$
\sum_{n=0}^{m-1}\left(\frac{1}{d}\right)^{n} u_{L\left(\omega^{q}\right)} \circ \Lambda^{n}(R) .
$$

So we have proved the lemma for $R \in \mathscr{C}_{k-q+1}$ smooth. In particular, for such an $R$, Corollary 3.1.7 gives that a super-potential of $L^{m}\left(\omega^{q}\right)$ is given on $R$ by:

$$
\sum_{n=0}^{m-1}\left(\frac{1}{d}\right)^{n} u_{L\left(\omega^{q}\right)} \circ \Lambda_{n}(R) .
$$

Now, assume $R$ is only $\left(f^{m}\right)_{*}$-admissible. Lemma 3.1 .8 implies that $R$ is $\left(f^{n}\right)^{*}$ admissible then for all $n$ with $0 \leq n \leq m$. We consider a sequence of smooth current $\left(R_{j}\right)$ of $\mathscr{C}_{k-q+1}$ converging to $R$ in the Hartogs' sense. Then $\Lambda^{n}\left(R_{j}\right)$ is smooth near $I^{+}$for $0 \leq n \leq m-1$ by (7) and every term in the super-potential of $L^{m}\left(\omega^{q}\right)$ is continuous for the Hartogs' convergence, that gives that:

$$
u_{L^{m}\left(\omega^{q}\right)}(R)=\sum_{n=0}^{m-1}\left(\frac{1}{d}\right)^{n} u_{L\left(\omega^{q}\right)} \circ \Lambda_{n}(R) .
$$

Again, Lemma 3.1 .8 gives that $\Lambda_{n}(R)=\Lambda^{n}(R)$ for all $n \leq m-1$ if $R$ is $\left(f^{m}\right)_{*^{-}}$ admissible so the lemma is proved.

REMARK 3.2.3. - The equality (8) also stands for any current $R \in \mathscr{C}_{k-q+1}$ such that $\Lambda^{n}(R)$ is smooth near $I^{+}$for $0 \leq n \leq m-1$. Indeed such currents are $\left(f^{m}\right)_{*}$ admissible: every term of the form $\mathcal{U}_{L\left(\omega^{q}\right)} \circ \Lambda^{n}(R)$ is finite for $n \leq m-1$ and depends continuously on $R$ for the Hartogs' convergence, so we have by (8) that a superpotential of $L^{m}\left(\omega^{q}\right)$ is finite at $R$ this means by symmetry of the super-potential that a super-potential of $R$ is finite at $L^{m}\left(\omega^{q}\right)$ hence $R$ is $\left(f^{m}\right)_{*}$-admissible.

Since the sequence of super-potential of $\left(L^{m}\left(\omega^{s}\right)\right)$ given by $(8)$ is decreasing, it is enough to show that it does not converge uniformly to $-\infty$ to show that it converges in the Hartogs' sense (see Proposition A.1.8). In [31], the authors prove that fact in the algebraically $q$-stable case with an additional assumption on the size of $\mathscr{C}^{+}$ (that fails in our case) using the fact that the sequence is bounded from below by the super-potential of any weak limit of the Cesarò mean of $\left(L^{m}\left(\omega^{q}\right)\right)$. Here the idea is to show that the convergence holds at the point $\left[I^{-}\right]_{\text {nor }}$.

We need the following estimate of $\mathcal{U}_{L(\omega)}$ for that. It is similar to Proposition 1.3 in [1] though our proof is simpler taking advantage of the fact that we are in $\mathbb{P}^{k}$. 
LEMMA 3.2.4. - Let $U_{L(\omega)}$ be a quasi-potential of $L(\omega)$. Then there exist constants $A>0, B, A^{\prime}>0, B^{\prime}$ such that:

$$
A \log \operatorname{dist}\left(x, I^{+}\right)-B \leq U_{L(\omega)}(x) \leq A^{\prime} \log \operatorname{dist}\left(x, I^{+}\right)+B^{\prime},
$$

for all $x$.

Proof of the lemma. - Let $P_{1}, \ldots, P_{k+1}$ be homogeneous polynomials of degree $d$ with no common factors such that $f=\left[P_{1}: \ldots: P_{k+1}\right]$. That way, $I^{+}=\left\{P_{1}=\cdots=\right.$ $\left.P_{k+1}=0\right\}$. For an element $Z=\left(z_{1}, \ldots, z_{k+1}\right) \in \mathbb{C}^{k+1}$, we write $|Z|^{2}=\left|z_{1}\right|^{2}+$ $\cdots+\left|z_{k+1}\right|^{2}$. Let $\pi: \mathbb{C}^{k+1} \rightarrow \mathbb{P}^{k}$ denote the canonical projection and we write $F=\left(P_{1}, \ldots, P_{k+1}\right)$. Then, we have that:

$$
\pi^{*}(L(\omega)-\omega)=d d^{c}\left(\frac{1}{d} \log |F|^{2}-\log |Z|^{2}\right) .
$$

Observe that the qpsh function $d^{-1} \log |F|^{2}-\log |Z|^{2}$ is well defined on $\mathbb{P}^{k}$ since it does not depend on the choice of coordinates. So we can write that $U_{L(\omega)}=d^{-1} \log |F|^{2}-$ $\log |Z|^{2}$. So in $\mathbb{P}^{k}$, the singularities of $U_{L(\omega)}$ come from the terms in $\log |F|^{2}$. In the open set of $\mathbb{C}^{k+1}$ defined by $1-\varepsilon<|Z|<1+\varepsilon$, we have that the map $F(Z)$ is equal to $(0, \ldots, 0) \in \mathbb{C}^{k+1}$ exactly in $\pi^{-1}\left(I^{+}\right)$. Using Łojasiewicz Theorem (Chapter IV Theorem 7 in [46]), that provide us with two constants $\alpha>0$ and $C>0$ such that on $|Z|=1$ we have:

$$
|F(Z)| \geq C\left(\operatorname{dist}\left(Z, \pi^{-1}\left(I^{+}\right)\right)\right)^{\alpha} .
$$

Now from the fact that the projection $\pi$ is Lipschitz in $|Z|=1$ and the above bound, we have constants $A>0, B$ such that:

$$
U_{L(\omega)} \geq A \log \operatorname{dist}\left(., I^{+}\right)-B .
$$

For the other inequality, we work in a chart of $\mathbb{P}^{k}$ where we let $z$ be the coordinate. Let $V$ be a relatively compact open set in the chart. Observe that it is sufficient to prove the upper bound in $V$. For $y \in I^{+}$in the chart, we have that $|F(z)|^{2}=$ $\left.|| F(z)\right|^{2}-|F(y)|^{2} \mid$ is less than $C^{\prime} \operatorname{dist}(z, y)$. Taking the infimum over all such $y$, we get that $|F(z)|^{2}$ is less than $C^{\prime} \operatorname{dist}\left(z, I^{+}\right)$. Taking the logarithm gives the estimate in $V$ and the lemma follows.

Proof of the theorem. - Since $f$ satisfies $(7), \Lambda^{n}\left(\left[I^{-}\right]_{\text {nor }}\right) \in \mathscr{C}_{k-s+1}$ is smooth in a neighborhood of $I^{+}$for all $n$ (hence $\left[I^{-}\right]_{\text {nor }}$ is $\left(f^{n}\right)_{*}$-admissible for all $n$ ). So $\Lambda^{n}\left(\left[I^{-}\right]_{\text {nor }}\right)=\left[f^{n}\left(I^{-}\right)\right]_{\text {nor }}$ (counting the multiplicity). Hence a super-potential of $L^{m}\left(\omega^{s}\right)$ is given on $\left[I^{-}\right]_{\text {nor }}$ by $(8)$ :

$$
\mathcal{U}_{L^{m}\left(\omega^{s}\right)}\left(\left[I^{-}\right]_{\text {nor }}\right)=\sum_{n=0}^{m-1}\left(\frac{1}{d}\right)^{n} \mathcal{U}_{L\left(\omega^{s}\right)}\left(\Lambda^{n}\left(\left[I^{-}\right]_{\text {nor }}\right)\right) .
$$


In other words:

$$
\mathcal{U}_{L^{m}\left(\omega^{s}\right)}\left(\left[I^{-}\right]_{\text {nor }}\right)=\sum_{n=0}^{m-1}\left(\frac{1}{d}\right)^{n} \mathcal{U}_{L\left(\omega^{s}\right)}\left(\left[f^{n}\left(I^{-}\right)\right]_{\text {nor }}\right) .
$$

Recall again that $L\left(\omega^{s}\right)=L\left(\omega^{s-1}\right) \wedge L(\omega)$ in the sense of current by Corollary 4.11 in [14, Chapter III]. So, in particular by Lemma A.2.1, a super-potential of $U_{L\left(\omega^{s}\right)}$ is given by:

$$
\mathcal{U}_{L\left(\omega^{s}\right)}(R)=\mathcal{U}_{L(\omega)}\left(L\left(\omega^{s-1}\right) \wedge R\right)+\mathcal{U}_{L\left(\omega^{s-1}\right)}(\omega \wedge R)
$$

on currents $R \in \mathscr{C}_{k-s+1}$ such that $L\left(\omega^{s-1}\right)$ and $R$ are wedgeable. A straightforward induction gives that a super-potential of $L\left(\omega^{s}\right)$ is given by:

$$
\sum_{0 \leq j \leq s-1} u_{L(\omega)}\left(\omega^{j} \wedge L(\omega)^{s-1-j} \wedge R\right)
$$

on currents $R \in \mathscr{C}_{k-s+1}$ such that $L\left(\omega^{s-1}\right)$ and $R$ are wedgeable (since $\omega^{j} \wedge L\left(\omega^{s-1-j}\right)$ is more H-regular than $L\left(\omega^{s-1}\right)$, we have that $\omega^{j} \wedge L(\omega)^{s-1-j}$ and $R$ are wedgeable by Lemma A.1.14). In particular, $L\left(\omega^{s-1}\right)$ and $\Lambda^{n}\left(\left[I^{-}\right]_{\text {nor }}\right)$ are wedgeable by Definition $3.1 .9\left((7)\right.$ is enough for that since $L\left(\omega^{s-1}\right)$ is smooth near $\left.f^{n}\left(I^{-}\right)\right)$so we can take $R=\Lambda^{n}\left(\left[I^{-}\right]_{\text {nor }}\right)$ in the previous formula.

We want to show that for $0 \leq j \leq s-1$, the following series is convergent:

$$
\sum_{n \geq 0}\left(\frac{1}{d}\right)^{n} \mathcal{U}_{L(\omega)}\left(\omega^{j} \wedge L(\omega)^{s-1-j} \wedge \Lambda^{n}\left(\left[I^{-}\right]_{\mathrm{nor}}\right)\right) .
$$

By Lemma A.1.16, the term of the series can be rewritten as:

$$
a_{j, n}=\operatorname{vol}\left(I^{-}\right)^{-1} \frac{1}{d^{s n}} \int_{f^{n}\left(I^{-}\right)} U_{L(\omega)} \omega^{j} \wedge L(\omega)^{s-1-j},
$$

since $\Lambda^{n}\left(\left[I^{-}\right]_{\text {nor }}\right)=\left[f^{n}\left(I^{-}\right)\right]_{\text {nor }}=\operatorname{vol}\left(I^{-}\right)^{-1} d^{-(s-1) n}\left[f^{n}\left(I^{-}\right)\right]$(observe that the form $U_{L(\omega)} \omega^{j} \wedge L(\omega)^{s-1-j}$ is smooth on $f^{n}\left(I^{-}\right)$so this integral makes sense). So in particular, Definition 3.1.9 is equivalent to the fact that the series converges for $j=0$. We prove that the series converges for $j>0$ by induction.

So let $j>0$ be given such that the above series converges for $j-1$. Using $L(\omega)=$ $d d^{c} U_{L(\omega)}+\omega$, we write:

$$
\omega^{j-1} \wedge L(\omega)^{s-j}=\omega^{j} \wedge L(\omega)^{s-1-j}+d d^{c} U_{L(\omega)} \wedge \omega^{j-1} \wedge L(\omega)^{s-1-j} .
$$

So replacing in (12), we see that:

$$
a_{j-1, n}=a_{j, n}+\operatorname{vol}\left(I^{-}\right)^{-1} \frac{1}{d^{s n}} \int_{f^{n}\left(I^{-}\right)} U_{L(\omega)} d d^{c} U_{L(\omega)} \wedge \omega^{j-1} \wedge L(\omega)^{s-1-j} .
$$

By Stokes, we have that the last integral is equal to:

$$
-\int_{f^{n}\left(I^{-}\right)} d U_{L(\omega)} \wedge d^{c} U_{L(\omega)} \wedge \omega^{j-1} \wedge L(\omega)^{s-1-j},
$$


which is non positive because $\omega^{j-1} \wedge L(\omega)^{s-1-j}$ is positive. That means that:

$$
a_{j-1, n} \leq a_{j, n}
$$

Since $a_{j, n}<0$ (because $U_{L(\omega)}<0$ ), we have the convergence of the series for $j$. That concludes the induction.

Proposition A.1.8 gives the convergence in the Hartogs' sense to $T_{s}^{+}$.

The previous proof gives in fact the corollary:

COROLlaRY 3.2.5. - The convergence of the series giving $\mathcal{U}_{T_{s}^{+}}\left(\left[I^{-}\right]_{\mathrm{nor}}\right)$ is equivalent to the convergence of the first series in 3.1.9:

$$
\sum_{n}\left(\frac{1}{d^{s}}\right)^{n}\left(\operatorname{vol}\left(I^{-}\right)\right)^{-1} \int_{f^{n}\left(I^{-}\right)} U_{L(\omega)} L\left(\omega^{s-1}\right)>-\infty .
$$

REMARK 3.2.6. - Using the same argument for $f^{n}\left(I^{-}\right)$instead of $I^{-}$shows that the super-potentials of the current $T_{s}^{+}$are in fact finite at every $\left[f^{n}\left(I^{-}\right)\right]_{\text {nor }}$.

Observe also that the Green current $T_{s}^{+}\left(f^{n}\right)$ of $f^{n}$ is well defined and equal to $T_{s}^{+}$.

REMARK 3.2.7. - We will see in the next section how to construct the Green current of order $q$ for $q \leq s$ as $T_{q}^{+}=\lim L^{n}\left(\omega^{q}\right)$ (see Proposition 3.4.6). We will need to construct the equilibrium measure for that first.

We now prove that if a map satisfies Hypothesis 3.1.10 then it is in $\mathscr{B}$. In fact, for such a map, we can construct directly the Green currents of order $\leq s$, in the general case, such result will only be proved in Section 3.4.

Theorem 3.2.8. - Any map satisfying Hypothesis 3.1.10 is in $\mathcal{B}$. For those maps, we have for $q \leq s$ that $\left(L^{m}\left(\omega^{q}\right)\right)$ converges in the Hartogs' sense to the Green current of order $q$ of $f$ that we denote by $T_{q}^{+}$.

Proof. - Take $f$ satisfying Hypothesis 3.1 .10 and let $q \leq s$. We consider $R_{I^{-}} \in$ $\mathscr{C}_{k-q+1}$ any positive closed current with support in $I^{-}$(for example $\omega^{s-q} \wedge\left[I^{-}\right]_{\text {nor }}$ ). Then $\Lambda^{j}(R)$ is smooth near $I^{+}$for all $j \leq m-1$ (it is zero there), so we can apply (8):

$$
u_{L^{m}\left(\omega^{q}\right)}\left(R_{I^{-}}\right)=\sum_{n=0}^{m-1}\left(\frac{1}{d}\right)^{n} u_{L\left(\omega^{q}\right)}\left(\Lambda^{n}\left(R_{I^{-}}\right)\right) .
$$

Using (11), we see that

$$
U_{L\left(\omega^{q}\right)}\left(\Lambda^{n}\left(R_{I^{-}}\right)\right)=\left\langle U_{L(\omega)}, S_{q} \wedge \Lambda^{n}\left(R_{I^{-}}\right)\right\rangle,
$$

where $S_{q}=\sum_{0 \leq j \leq q-1} \omega^{j} \wedge L(\omega)^{q-1-j}$ is smooth near $f^{n}\left(I^{-}\right)$and is of mass $q$. The measure $S_{q} \wedge \Lambda^{n}\left(R_{I^{-}}\right)$is of mass $q$ with support in $f^{n}\left(I^{-}\right)$. By Lemma 3.2.4, the function $U_{L(\omega)}$ is greater than $A \log \operatorname{dist}\left(I^{+}, f^{n}\left(I^{-}\right)\right)-B$ on $f^{n}\left(I^{-}\right)$. Hypothesis 3.1 .10 
implies exactly the convergence of the series giving $\mathcal{U}_{L^{m}\left(\omega^{q}\right)}\left(R_{I^{-}}\right)$. That concludes the proof by Proposition A.1.8.

ThEOREM 3.2.9. - The current $T_{s}^{+}$is $f^{*}$-invariant, that is $L\left(T_{s}^{+}\right)$is well defined and equal to $T_{s}^{+}$.

Furthermore, $T_{s}^{+}$is the most $H$-regular current which is $f^{*}$-invariant in $\mathscr{C}_{s}$. In particular, $T_{s}^{+}$is extremal in the set of $f^{*}$-invariant currents of $\mathscr{C}_{s}$.

Proof. - Recall from the appendix that a current $T$ is $f^{*}$-admissible if there exists a current $R_{0}$ which is smooth on a neighborhood of $I^{+}$such that the super-potentials of $T$ are finite at $\Lambda\left(R_{0}\right)$. For such $T, f^{*}(T)$ is well defined and if $\left(T_{n}\right)$ is a sequence of current converging in the Hartogs' sense to $T$ then $T_{n}$ is $f^{*}$-admissible and $f^{*}\left(T_{n}\right)$ converges to $f^{*}(T)$ in the Hartogs' sense.

In our case, we take for $R_{0}$ the current $\left[I^{-}\right]_{\text {nor }}$ which is smooth near $I^{+}$. Then by Remark 3.2.6, the super-potentials of $T_{s}^{+}$are finite at $\Lambda\left(\left[I^{-}\right]_{\text {nor }}\right)=\left[f\left(I^{-}\right)\right]_{\text {nor }}$. So the current $L\left(T_{s}^{+}\right)$is well defined. Now, we have that $\left(L^{n+1}\left(\omega^{s}\right)\right)_{n}=\left(L\left(L^{n}\left(\omega^{s}\right)\right)\right)_{n}$ converges in the Hartogs' sense to $T_{s}^{+}$and to $L\left(T_{s}^{+}\right)$so that $T_{s}^{+}$is indeed invariant.

Let $\mathcal{U}_{T_{s}^{+}}$be the super-potential of $T_{s}^{+}$defined as:

$$
u_{T_{s}^{+}}=\sum_{n=0}^{\infty}\left(\frac{1}{d}\right)^{n} u_{L\left(\omega^{s}\right)} \circ \Lambda^{n}
$$

on smooth forms in $\mathscr{C}_{k-s+1}$. Then composing (13) with $\Lambda$ gives that on smooth forms in $\mathscr{C}_{k-s+1}$ :

$$
\mathcal{U}_{T_{s}^{+}}=d^{-1} \mathcal{U}_{T_{s}^{+}} \circ \Lambda+\mathcal{U}_{L\left(\omega^{s}\right)} .
$$

By iteration, we have that on smooth forms in $\mathscr{C}_{k-s+1}$ :

$$
u_{T_{s}^{+}}=\sum_{n=0}^{m-1}\left(\frac{1}{d}\right)^{n} u_{L\left(\omega^{s}\right)} \circ \Lambda^{n}+\left(\frac{1}{d}\right)^{m} u_{T_{s}^{+}} \circ \Lambda^{m} .
$$

By Theorem 3.2.1, that implies by difference that:

$$
\left(\frac{1}{d}\right)^{m} u_{T_{s}^{+}} \circ \Lambda^{m}
$$

goes to zero on smooth forms in $\mathscr{C}_{k-s+1}$.

Now, let $S$ be a $f^{*}$-invariant current in $\mathscr{C}_{s}$ such that there are constants $A>0$ and $B$ satisfying $A \mathcal{U}_{T_{s}^{+}}+B \leq \mathcal{U}_{S}<0$ for some super-potential $\mathcal{U}_{S}$. Then on smooth forms in $\mathscr{C}_{k-s+1}$, a super-potential $\mathcal{U}_{L^{m}(S)}$ of $L^{m}(S)=S$ is given by:

$$
\sum_{n=0}^{m-1}\left(\frac{1}{d}\right)^{n} u_{L\left(\omega^{s}\right)} \circ \Lambda^{n}+\left(\frac{1}{d}\right)^{m} \mathcal{U}_{S} \circ \Lambda^{m} .
$$


Since $\left(\frac{1}{d}\right)^{m} u_{T_{s}^{+}} \circ \Lambda^{m}$ goes to zero on smooth forms in $\mathscr{C}_{k-s+1}$, our hypothesis implies that $\left(\frac{1}{d}\right)^{m} U_{S} \circ \Lambda^{m}$ also goes to zero on smooth forms in $\mathscr{C}_{k-s+1}$. In particular, a super-potential of $S$ is given on smooth forms in $\mathscr{C}_{k-s+1}$ by:

$$
\sum_{n=0}^{\infty}\left(\frac{1}{d}\right)^{n} u_{L\left(\omega^{s}\right)} \circ \Lambda^{n}
$$

Now, using the fact that two currents having the same super-potential on smooth forms are in fact equal we deduce that $T_{s}^{+}=S$.

In particular, for $A=1$, we obtain that $T_{s}^{+}$is the most $H$-regular current which is $f^{*}$-invariant. It is extremal in the set of $f^{*}$-invariant currents of $\mathscr{C}_{s}$ since if not we could write $T_{s}^{+}=t S_{1}+(1-t) S_{2}$ with $S_{1}$ and $S_{2}$ two $f^{*}$-invariant currents in $\mathscr{C}_{s}$. Take $M$ small enough so that the super-potentials $\mathcal{U}_{1}, \mathcal{U}_{2}$ and $\mathcal{U}_{T_{s}^{+}}$of $S_{1}, S_{2}$ and $T_{s}^{+}$of same mean $M$ are negative. Observe that $\mathcal{U}_{T_{s}^{+}}=t \mathcal{U}_{1}+(1-t) \mathcal{U}_{2}$ so that $t^{-1} \mathcal{U}_{T_{s}^{+}} \leq \mathcal{U}_{1}$. Then we can apply the previous result for $A=t^{-1}$ and it follows that $S_{1}=T$ (similarly $S_{2}=T$ ).

In the previous proof, we have obtained:

COROLLARY 3.2.10. - Let $\mathcal{U}_{T_{s}^{+}}$be the super-potential of $T_{s}^{+}$defined on smooth forms by:

$$
u_{T_{s}^{+}}=\sum_{n=0}^{\infty}\left(\frac{1}{d}\right)^{n} u_{L\left(\omega^{s}\right)} \circ \Lambda^{n}
$$

Then we have that:

$$
\left(\frac{1}{d}\right)^{m} U_{T_{s}^{+}} \circ \Lambda^{m}
$$

goes to zero on smooth forms.

The current $L(\omega)^{s+1}$ is a well defined element of $\mathscr{C}_{s+1}$ and we have that

$$
d d^{c} U_{L(\omega)} \wedge L(\omega)^{s}+\omega \wedge L(\omega)^{s}=L(\omega)^{s+1}
$$

by Corollary 4.11 Chapter III in [14]. Observe that it is not true though that $L\left(\omega^{s+1}\right)=L(\omega)^{s+1}$. Indeed, $f^{*}\left(\omega^{s+1}\right)$ is a well defined form (of mass $\delta^{k-s-1}$ ) which is in $L^{1}$ hence that does not give mass to algebraic sets of dimension $\leq k-1$. But $f^{*}(\omega)^{s+1}$ is a smooth form outside $I^{+}$which coincides with $f^{*}\left(\omega^{s+1}\right)$ there. So we have by Siu's Theorem that:

$$
f^{*}(\omega)^{s+1}=\sum_{i} a_{i}\left[I_{i}^{+}\right]+f^{*}\left(\omega^{s+1}\right)
$$

where the sum goes over all the irreducible components $I_{i}^{+}$of $I^{+}$and where the $a_{i}$ are positive numbers. Observe that this formula is related to King's formula (see [14] Chapter III proposition 8.18). In particular, one has that:

$$
f^{*}(\omega)^{s+1} \leq C\left[I^{+}\right]+f^{*}\left(\omega^{s+1}\right)
$$


for $C>0$ large enough. Similarly, one has that $f_{*}(\omega)^{k-s+1}$ is well defined and satisfies:

$$
f_{*}(\omega)^{k-s+1} \leq C\left[I^{-}\right]+f_{*}\left(\omega^{k-s+1}\right) .
$$

The following proposition is useful in the construction of the equilibrium measure.

Proposition 3.2.11. - The super-potentials of $T_{s}^{+}$are finite at $\omega^{j} \wedge \Lambda(\omega)^{k-s+1-j}$ for all $k-s+1 \geq j \geq 0$.

Proof. - If two currents $S_{1}$ and $S_{2}$ in $\mathscr{C}_{r}$ satisfies $S_{1} \leq c S_{2}$ for a constant $c>0$ then the super-potentials of $S_{1}$ and $S_{2}$ of mean 0 satifies $\mathcal{U}_{S_{1}} \geq c \mathcal{U}_{S_{2}}+c^{\prime}$ for some constant $c^{\prime}$. In particular, the super-potentials of $S_{1}$ are finite wherever $\mathcal{U}_{S_{2}}$ is.

Now, we have that the super-potentials of $T_{s}^{+}$are finite at $\left[I^{-}\right]_{\text {nor }}$. Since $T_{s}^{+}$is $f^{*}$-admissible, its super-potential are finite at every point of the form $\Lambda(S)$ for $S \in$ $\mathscr{C}_{k-s+1}$ smooth near $I^{+}$. So they are also finite at $\Lambda\left(\omega^{k-s+1}\right)$. The affinity of the super-potentials of $T_{s}^{+}$implies that they are finite at $\left(C\left[I^{-}\right]+f_{*}\left(\omega^{k-s+1}\right)\right)_{\text {nor }}$. So the super-potential of $T_{s}^{+}$are finite at $\left(f_{*}(\omega)^{k-s+1}\right)$ nor. Since for $j \geq 0$, the current $\omega^{j} \wedge \Lambda(\omega)^{k-s+1-j}$ is more H-regular than $\Lambda(\omega)^{k-s+1}$, we have that the super-potentials of $T_{s}^{+}$are finite at $\omega^{j} \wedge \Lambda(\omega)^{k-s+1-j}$ (we use the symmetry of the super-potential : $\left.\mathcal{U}_{T_{s}^{+}}\left(\omega^{j} \wedge \Lambda(\omega)^{k-s+1-j}\right)=\mathcal{U}_{\omega^{j} \wedge \Lambda(\omega)^{k-s+1-j}}\left(T_{s}^{+}\right)\right)$.

Corollary 3.2.12. - The current $T_{s}^{+}$gives no mass to $I^{-}$(nor $I^{+}$by dimension's argument).

Proof. - From above, the super-potentials of $T_{s}^{+}$are finite at $\Lambda(\omega) \wedge \omega^{k-s} \in \mathscr{C}_{k-s+1}$. Observe that for two currents $R$ and $S$ in $\mathscr{C}_{r}$ and $\mathscr{C}_{s}$ with $r+s \leq k$, then:

$$
\mathcal{U}_{R}\left(S \wedge \omega^{k+1-r-s}\right)=\mathcal{U}_{R \wedge \omega^{k+1-r-s}}(S)=\mathcal{U}_{S}\left(R \wedge \omega^{k+1-r-s}\right)
$$

where all the super-potentials are of same mean.

So for $R=T_{s}^{+}$and $S=\Lambda(\omega)$, we get that the super-potentials of $\Lambda(\omega)$ are finite at $T_{s}^{+} \wedge \omega^{k-s}$. This is equivalent to the fact that the quasi-potential $U_{\Lambda(\omega)}$ is integrable with respect to the measure $T_{s}^{+} \wedge \omega^{k-s}$. In other words:

$$
\int U_{\Lambda(\omega)} \omega^{k-s} \wedge T_{s}^{+}
$$

is finite. Applying Lemma 3.2.4 to $f^{-1}$ shows that the singularities of $U_{\Lambda(\omega)}$ are in $\log \operatorname{dist}\left(x, I^{-}\right)$so $T_{s}^{+}$gives no mass to $I^{-}$.

REMARK 3.2.13. - The quantity $\mathcal{U}_{T_{s}^{+}}\left(\left[I^{-}\right]_{\text {nor }}\right)$ is related to a generalized Lelong number ([13], see also [9] for generalized Lelong numbers in dynamics). Let us explain this point. From the previous proposition, we have that the super-potentials of $T_{s}^{+}$ are finite at $\Lambda(\omega)^{k-s+1}$. 
We define the Lelong number of $T_{s}^{+}$associated to the function $U_{\Lambda(\omega)}$ as:

$$
\nu\left(T_{s}^{+}, U_{\Lambda(\omega)}\right)=\lim _{r \rightarrow-\infty} \int_{\left\{U_{\Lambda(\omega)}<r\right\}} T_{s}^{+} \wedge \Lambda(\omega)^{k-s} .
$$

The previous current is well defined by the theory of super-potentials: the superpotentials of $T_{s}^{+}$are finite at $\omega \wedge \Lambda(\omega)^{k-s}$ which means that $T_{s}^{+}$and $\Lambda(\omega)^{k-s}$ are wedgeable and their wedge product is well defined by Definition A.1.13.

As in formula (11), we have that a super-potential of $\Lambda(\omega)^{k-s+1}$ is given by:

$$
\sum_{0 \leq j \leq k-s} \mathcal{u}_{\Lambda(\omega)}\left(\omega^{j} \wedge \Lambda(\omega)^{k-s-j} \wedge R\right),
$$

on currents $R \in \mathscr{C}_{s}$ such that $\Lambda(\omega)^{k-s}$ and $R$ are wedgeable, so in particular for $R=T_{s}^{+}$by the previous proposition. Since the super-potentials of $T_{s}^{+}$are finite at $\Lambda(\omega)^{k-s+1}$, that implies that every term in the previous sum is finite. So we have in particular that:

$$
\mathcal{U}_{\Lambda(\omega)}\left(\Lambda(\omega)^{k-s} \wedge T_{s}^{+}\right)
$$

is finite. That means that the quasi-potential $U_{\Lambda(\omega)}$ is integrable with respect to the measure $\Lambda(\omega)^{k-s} \wedge T_{s}^{+}$. So we can use the bound:

$$
\begin{aligned}
\int_{\left\{U_{\Lambda(\omega)}<r\right\}} T_{s}^{+} \wedge \Lambda(\omega)^{k-s} & \leq \frac{1}{r} \int_{\left\{U_{\Lambda(\omega)}<r\right\}} U_{\Lambda(\omega)} T_{s}^{+} \wedge \Lambda(\omega)^{k-s} \\
& \leq \frac{1}{r} \int_{\mathbb{P}^{k}} U_{\Lambda(\omega)} T_{s}^{+} \wedge \Lambda(\omega)^{k-s} .
\end{aligned}
$$

So we have that:

$$
\nu\left(T_{s}^{+}, U_{\Lambda(\omega)}\right)=0 .
$$

This is a generalization of the fact that a psh function finite at the point $x$ has zero Lelong number at $x$.

A classical question in complex dynamics is to ask by what $\omega^{s}$ can be replaced. In other words, what are the currents $T$ in $\mathscr{C}_{s}$ such that $L^{m}(T) \rightarrow T_{s}^{+}$? The following proposition and theorem give partial results toward this direction.

Proposition 3.2.14. - Let $\left(T_{m}\right) \in \mathscr{C}_{s}$ be a sequence of currents such that a superpotential $\mathcal{U}_{T_{m}}$ of $T_{m}$ satisfies $\left\|U_{T_{m}}\right\|_{\infty}=o\left(d^{m}\right)$. Then $L^{m}\left(T_{m}\right) \rightarrow T_{s}^{+}$in the Hartogs' sense.

Proof. - Recall first that if $T \in \mathscr{C}_{s}$ has bounded super-potential it is $\left(f^{n}\right)^{*}$-admissible (its super-potential is in particular bounded at the point $\Lambda^{n}\left(\omega^{k-s+1}\right)$ ). So the sequence 
of pull-back is well defined by Lemma 3.1.8. Using Proposition A.1.18 and (8), a superpotential of $L^{m}\left(T_{m}\right)$ is given on smooth currents in $\mathscr{C}_{k-s+1}$ by:

$$
\mathcal{U}_{L^{m}\left(T_{m}\right)}=\sum_{n=0}^{m-1}\left(\frac{1}{d}\right)^{n} \mathcal{U}_{L\left(\omega^{s}\right)} \circ \Lambda^{n}+\left(\frac{1}{d}\right)^{m} \mathcal{U}_{T_{m}} \circ \Lambda^{m} .
$$

By Theorem 3.2.1 we know that the series $\sum_{n=0}^{m-1}\left(\frac{1}{d}\right)^{n} U_{L\left(\omega^{s}\right)} \circ \Lambda^{n}$ converges in the Hartogs' sense to $\mathcal{U}_{T_{s}^{+}}$. The hypothesis on $\left(T_{m}\right)$ implies that $\left(\frac{1}{d}\right)^{m} \mathcal{U}_{T_{m}} \circ \Lambda^{m}=o(1)$ goes to 0 uniformly on smooth form. Since the control is uniform we have that $\left(\left|\mathcal{U}_{L^{m}\left(T_{m}\right)}-\mathcal{U}_{L^{m}\left(\omega^{s}\right)}\right| \rightarrow 0\right)$, and the convergence is in the Hartogs' sense and we can conclude by Proposition A.1.7.

We also have the following result. We believe the proof is of interest although the result is essentially already known. We refer the reader to [50] for the case $q=1$ and also [27] for the general case. See the Appendix for the notion of super-polarity.

Theorem 3.2.15. - There exists a super-polar set $P$ of $\mathscr{C}_{s}$ such that for $S \in \mathscr{C}_{s} \backslash P$, $L^{m}(S)$ is well defined and converges to $T_{s}^{+}$.

Proof. - The set of currents $S \in \mathscr{C}_{s}$ such that $S$ is not $\left(f^{m}\right)^{*}$-admissible is superpolar since it is contained in the set of currents such that $\mathcal{U}_{S}\left(\Lambda^{m}\left(\omega^{k-s+1}\right)\right)=-\infty$. Now, since a countable union of super-polar set is super-polar, we have that outside a super-polar set of $\mathscr{C}_{s}, S$ is $\left(f^{m}\right)^{*}$-admissible and so $L^{m}(S)$ is well defined by Lemma 3.1.8.

As above, a super-potential $U_{L^{m}(S)}$ of $L^{m}(S)$ is given on smooth forms by:

$$
\sum_{n=0}^{m-1}\left(\frac{1}{d}\right)^{n} \mathcal{u}_{L\left(\omega^{s}\right)} \circ \Lambda^{n}+\left(\frac{1}{d}\right)^{m} \mathcal{U}_{S} \circ \Lambda^{m},
$$

where $\mathcal{U}_{S}$ is a super-potential of $S$. For $\Omega \in \mathscr{C}_{k-s+1}$ smooth, consider the current $R(\Omega) \in \mathscr{C}_{k-s+1}$ defined by $R(\Omega):=\left(\sum_{m}\left(\frac{1}{d}\right)^{m} \Lambda^{m}(\Omega)\right)_{\text {nor. }}$. Let $P$ be the set of currents $S$ in $\mathscr{C}_{s}$ such that $\mathcal{U}_{S}\left(R\left(\omega^{k-s+1}\right)\right)=-\infty$, then $P$ is super-polar by definition. Observe that for $\Omega \in \mathscr{C}_{k-s+1}$ smooth, we have a constant $c_{\Omega}>0$ such that $R(\Omega) \leq c_{\Omega} R\left(\omega^{k-s+1}\right)$. In particular, for $S \notin P$, we have that $\mathcal{U}_{S}(R(\Omega))>-\infty$.

That implies that for any $\Omega$ smooth and $S \notin P$, the sequence $\mathcal{U}_{L^{m}(S)}(\Omega)$ converges to the value $\mathcal{U}_{T_{s}^{+}}(\Omega)$. Indeed, the fact that $\mathcal{U}_{S}(R(\Omega))$ is finite gives that:

$$
\left(\frac{1}{d}\right)^{m} u_{S} \circ \Lambda^{m}(\Omega)
$$

goes to 0 . So $\mathcal{U}_{L^{m}(S)}(\Omega)$ converges to $\mathcal{U}_{T_{s}^{+}}(\Omega)$. Then Proposition A.1.7 gives us that the sequence $L^{m}(S)$ converges in fact to $T_{s}^{+}$(maybe not in the Hartogs' sense). 
Of course, the above theorem is not optimal and it is conjectured that for $T$ the current of integration on a (very) generic algebraic set of dimension $k-s, L^{m}(T)$ goes to $T_{s}^{+}$(see in the endomorphisms case [5] and [21] for the case of measures and see [35] for the case of bidegree $(1,1)$ in $\mathbb{P}^{2}$ and [30] in $\left.\mathbb{P}^{k}\right)$. That is beyond the scope of this study.

Recall that we consider the critical sets $\mathscr{C}^{+}$(or $\left.\mathscr{C}(f)\right)$ and $\mathscr{C}^{-}\left(\right.$or $\left.\mathscr{C}\left(f^{-1}\right)\right)$ defined by:

$$
\begin{aligned}
& \mathscr{C}^{+}:=f^{-1}\left(I^{-}\right) \\
& \mathscr{C}^{-}:=\left(f^{-1}\right)^{-1}\left(I^{+}\right) .
\end{aligned}
$$

We have the proposition:

Proposition 3.2.16. - The current $T_{s}^{+}$does not give mass to the critical sets $\mathscr{C}^{-}$ and $\mathscr{C}^{+}$. In particular, the current $T_{s}^{+}$satisfies the equation:

$$
f_{*}\left(T_{s}^{+}\right)=\frac{1}{d_{s}} T_{s}^{+},
$$

in $\mathbb{P}^{k} \backslash \mathscr{C}^{-}$.

We first need the following lemma:

LEMma 3.2.17. - Let $\varphi$ be a smooth function. Then $f_{*}(\varphi)$ is in $L^{1}\left(T_{s}^{+} \wedge \Lambda\left(\omega^{k-s}\right)\right)$ and we have the identity:

$$
\int \varphi T_{s}^{+} \wedge \omega^{k-s}=\int f_{*}(\varphi) T_{s}^{+} \wedge \Lambda\left(\omega^{k-s}\right)
$$

Proof of the lemma. - First $\varphi$ is in $L^{1}\left(T_{s}^{+} \wedge \omega^{k-s}\right)$ and the quantity:

$$
\int \varphi T_{s}^{+} \wedge \omega^{k-s}
$$

depends continuously on $T_{s}^{+}$in the sense of currents.

On the other hand, $f_{*}(\varphi)$ is in $L^{1}\left(T_{s}^{+} \wedge \Lambda\left(\omega^{k-s}\right)\right)$ since it is smooth and uniformly bounded outside $I^{-}$which has no mass for $T_{s}^{+} \wedge \Lambda\left(\omega^{k-s}\right)$ (see Remark 3.2.13). Recall that $T_{s}^{+} \wedge \Lambda(\omega)^{k-s}$ is $f^{*}$-admissible by Remark 3.2.13 (hence $U_{\Lambda(\omega)}\left(\Lambda(\omega)^{k-s} \wedge T_{s}^{+}\right.$) is finite). So we have that

$$
\int f_{*}(\varphi) T_{s}^{+} \wedge \Lambda\left(\omega^{k-s}\right)=\int \varphi L\left(T_{s}^{+} \wedge \Lambda\left(\omega^{k-s}\right)\right),
$$

as this stands if $T_{s}^{+} \wedge \Lambda\left(\omega^{k-s}\right)$ was smooth and we can conclude by Hartogs' convergence. Now, applying Lemma A.2.2 to $f^{-1}$ and the invariance of $T_{s}^{+}$, we have that $L\left(T_{s}^{+} \wedge \Lambda\left(\omega^{k-s}\right)\right)=T_{s}^{+} \wedge \omega^{k-s}$. 
Proof of the proposition. - We consider $\mathscr{C}^{+}$first. Let $V_{\varepsilon}$ be a small neighborhood of $I^{+}$. Since $T_{s}^{+}$gives no mass to $I^{+}$we can assume that the mass of $V_{\varepsilon}$ for $T_{s}^{+}$is arbitrarily small. Let $W_{\alpha}$ be a small neighborhood of $\mathscr{C}^{+}$. Let $0 \leq \varphi \leq 1$ be a smooth function such that $\varphi=1$ in $W_{\alpha} \backslash V_{\varepsilon}, \varphi=0$ in $V_{2^{-1} \varepsilon}$ and $\varphi=0$ outside $W_{2 \alpha}$.

Then by the previous lemma:

$$
\begin{aligned}
\left\|W_{\alpha} \backslash V_{\varepsilon}\right\|_{T_{s}^{+}} & \leq \int \varphi T_{s}^{+} \wedge \omega^{k-s} \\
& \leq \int f_{*}(\varphi) T_{s}^{+} \wedge \Lambda\left(\omega^{k-s}\right) \\
& \leq \int_{f\left(W_{2 \alpha} \backslash V_{2-1}\right)} T_{s}^{+} \wedge \Lambda\left(\omega^{k-s}\right)
\end{aligned}
$$

Now, $f\left(W_{2 \alpha} \backslash V_{2^{-1} \varepsilon}\right)$ is a neighborhood $W$ of $I^{-}$that can be taken arbitrarily small by taking $\varepsilon$ and $\alpha$ small enough. We have seen in Remark 3.2.13 that the quantity $\int_{W} T_{s}^{+} \wedge \Lambda(\omega)^{k-s}$ goes to the Lelong number $\nu\left(T_{s}^{+}, U_{\Lambda(\omega)}\right)$ which is equal to zero. Thus $\mathscr{C}^{+}$has no mass for $T_{s}^{+}$.

For $\mathscr{C}^{-}$, we write $T_{s}^{+}=T_{\mathscr{C}^{-}}+T^{\prime}$ where $T_{\mathscr{C}^{-}}$is a positive closed current with support in $\mathscr{C}^{-}$and $T^{\prime}$ is a positive closed current with no mass on $\mathscr{C}^{-}([\mathbf{5 2}])$. Both currents are $f^{*}$-admissible since $T_{s}^{+}$is. But $f^{*}\left(T_{\mathscr{C}^{-}}\right)$has support in $\mathscr{C}^{+}$which means it is equal to zero since $T_{s}^{+}=d_{s}^{-1}\left(f^{*}\left(T_{\mathscr{C}^{-}}\right)+f^{*}\left(T^{\prime}\right)\right)$ gives no mass to $\mathscr{C}^{+}$. That implies that $T_{\mathscr{C}^{-}}=0$ hence $T_{s}^{+}$has no mass on $\mathscr{C}^{-}$.

Now $f: \mathbb{P}^{k} \backslash \mathscr{C}^{+} \rightarrow \mathbb{P}^{k} \backslash \mathscr{C}^{-}$is a proper biholomorphism that we denote by $f_{1}$. If $\Theta$ is a smooth form in $\mathbb{P}^{k} \backslash \mathscr{C}^{-}$then using the invariance of $T_{s}^{+}$:

$$
\begin{aligned}
\left\langle\left(f_{1}\right)_{*}\left(T_{s}^{+}\right), \Theta\right\rangle & =\left\langle T_{s}^{+},\left(f_{1}\right)^{*}(\Theta)\right\rangle \\
& =\left\langle\frac{1}{d^{s}} f^{*}\left(T_{s}^{+}\right),\left(f_{1}\right)^{*}(\Theta)\right\rangle \\
& =\left\langle\frac{1}{d^{s}} T_{s}^{+}, f_{*}\left(\left(f_{1}\right)^{*}(\Theta)\right)\right\rangle .
\end{aligned}
$$

But $f_{*}\left(f_{1}\right)^{*}=\left(f_{1}\right)_{*}\left(f_{1}\right)^{*}=$ id so $f_{*}\left(\left(f_{1}\right)^{*}(\Theta)\right)=\Theta$ and the result follows.

REMARK 3.2.18. - In order to define $\Lambda\left(T_{s}^{+}\right)$, we need to add to the equation

$$
\left(f_{1}\right)_{*}\left(T_{s}^{+}\right)=\frac{1}{d_{s}} T_{s}^{+}
$$

a term with mass $d_{k-s}-d_{s}^{-1}$ and support in $\mathscr{C}^{-}$in order to obtain a current of mass $d_{k-s}$. For example, in the case of Hénon maps, we need to add a multiple of the current of integration on the line at infinity. In general, such choice is not clear as the hypersurface $\mathscr{C}^{-}$carries many positive closed currents of bidimension $(k-s, k-s)$ and they might be no way to add a current to the equation $\left(f_{1}\right)_{*}\left(T_{s}^{+}\right)=\frac{1}{d_{s}} T_{s}^{+}$in a continuous way. 
The previous corollary implies that the Green current is meaningful. For example, in the case of Hénon maps in $\mathbb{P}^{2}$, the set $\mathscr{C}^{-}$and $\mathscr{C}^{+}$are in fact $L_{\infty}$ (the line at infinity) which is totally invariant and the Green current $T^{+}$gives no mass to $L_{\infty}$.

We can now prove the following stronger result of extremality which implies strong ergodic properties (see Theorem 3.4.15). The inequalities between currents in $\mathscr{C}_{s}$ have to be understood in the strong sense.

Theorem 3.2.19. - The current $T_{s}^{+}$is extremal in $\mathscr{C}_{s}$, that is if there exists a $c>0$ and $S \in \mathscr{C}_{s}$ such that $S \leq c T_{s}^{+}$then $S=T_{s}^{+}$.

Proof. - Applying the previous results to $f^{n}$ gives that $T_{s}^{+}$gives no mass to the indeterminacy sets $I\left(f^{ \pm n}\right)$ and critical sets $\mathscr{C}^{ \pm n}$ of $f^{n}$ and $f^{-n}$ (recall that $T_{s}^{+}$is also the Green current of $\left.f^{n}\right)$. Let $S$ be as above, in particular $S$ gives no mass to the sets $I\left(f^{ \pm n}\right)$ and $\mathscr{C}\left(f^{ \pm n}\right)$ and $S$ is $\left(f^{n}\right)^{*}$-admissible for all $n$. By Lemma 3.1.8, $L^{n}(S)$ is well defined for all $n$ and equal to $L_{n}(S)\left(L_{n}\right.$ and $\Lambda_{n}$ are the normalized pull-pack and push-forward associated to $f^{n}$ ).

For $n>0$, we denote by $\Lambda_{n}^{\prime}$ the push forward operator $\left(f^{n}\right)_{*}$ from $\mathbb{P}^{k} \backslash \mathscr{C}\left(f^{n}\right)$ to $\mathbb{P}^{k} \backslash \mathscr{C}\left(f^{-n}\right)$.

The operator $\left(\Lambda_{n}^{\prime}\right)$ is positive. That and the previous proposition applied to $f^{n}$ imply that $\left(d_{s}\right)^{n}\left(\Lambda_{n}^{\prime}\right)(S) \leq c T_{s}^{+}$in $\mathbb{P}^{k} \backslash \mathscr{C}\left(f^{-n}\right)$. We denote by $S_{n}$ the trivial extension of $\left(d_{s}\right)^{n}\left(\Lambda_{n}^{\prime}\right)(S)$ over $\mathbb{P}^{k}$ : it does exist since the current $\left(d_{s}\right)^{n}\left(\Lambda_{n}^{\prime}\right)(S)$ is of bounded mass. We have $S_{n} \leq c T_{s}^{+}$in $\mathbb{P}^{k}$. In particular, $S_{n}$ is $\left(f^{*}\right)^{n}$-admissible. Using the same argument as in the previous proposition, we see that:

$$
\left(f^{n}\right)^{*}\left(S_{n}\right)=\left(d_{s}\right)^{n} S,
$$

outside $\mathscr{C}\left(f^{n}\right)$. Now $S$ has no mass on $\mathscr{C}\left(f^{n}\right)$ and $\left(f^{n}\right)^{*}\left(S_{n}\right)$ is less than $c\left(d^{s}\right)^{n} T_{s}^{+}$ (by positivity of the operator $\left(f^{n}\right)^{*}$ ) which implies that $\left(f^{n}\right)^{*}\left(S_{n}\right)$ also has no mass on $I\left(f^{n}\right) \cup \mathscr{C}\left(f^{n}\right)$. So we have:

$$
\left(f^{n}\right)^{*}\left(S_{n}\right)=\left(d_{s}\right)^{n} S,
$$

on $\mathbb{P}^{k}$. In particular, $S_{n}$ has mass 1 . We have that $L_{n}\left(S_{n}\right)=S$ and since $S_{n}$ is $\left(f^{*}\right)^{n}$-admissible we have $L_{n}\left(S_{n}\right)=L^{n}\left(S_{n}\right)$ by Lemma 3.1.8.

For $R \in C^{k-s+1}$ smooth, let $U_{S_{n}}, \mathcal{U}_{T_{s}^{+}, 0}$ and $\mathcal{U}_{\Lambda^{j}(R)}$ be the super-potential of $S_{n}$, $T_{s}^{+}$and $\Lambda^{j}(R)$ of mean 0. We have from Proposition A.1.18 and (8) that a superpotential $U_{L^{n}\left(S_{n}\right)}$ of $L^{n}\left(S_{n}\right)=S$ on smooth forms is given by:

$$
\sum_{j=0}^{n-1}\left(\frac{1}{d}\right)^{j} u_{L\left(\omega^{s}\right)} \circ \Lambda^{j}+\left(\frac{1}{d}\right)^{n} u_{S_{n}} \circ \Lambda^{n} .
$$

Recall that there exists a $M>0$ that does not depend on neither $R$ nor $n$ such that $U_{\Lambda^{n}(R)}-M$ is negative and $\mathcal{U}_{S_{n}} \leq M$. More precisely, by Proposition A.1.1, 
there exists a quasi-potential $U_{\Lambda^{n}(R)}$ of $\Lambda^{n}(R)$ such that $U_{\Lambda^{n}(R)}-M \omega^{k-s}$ is negative $\left(U_{\Lambda^{n}(R)}\right.$ is a quasi-potential of $\Lambda^{n}(R)$ of mean 0$)$. Then, we have that:

$$
\left(\mathcal{U}_{\Lambda^{n}(R)}-M\right)\left(S_{n}\right) \geq c\left(\mathcal{U}_{\Lambda^{n}(R)}-M\right)\left(T_{s}^{+}\right) .
$$

Indeed if $S_{n}$ and $T_{s}^{+}$were smooth, it would follow from the fact that $U_{\Lambda^{n}(R)}-M \omega^{k-s}$ is negative and that $S_{n} \leq c T_{s}^{+}$. The result follows then by Hartogs' convergence: observe that the regularization is obtained by a mean of the composition over the automorphisms of $\mathbb{P}^{k}$ thus the approximations $S_{n}^{\prime}$ and $T_{s}^{\prime+}$ of $S_{n}$ and $T_{s}^{+}$also satisfy $S_{n}^{\prime} \leq c T_{s}^{\prime+}$.

So we have the estimates:

$$
\begin{aligned}
\mathcal{U}_{S_{n}}\left(\Lambda^{n}(R)\right) & =\mathcal{U}_{\Lambda^{n}(R)}\left(S_{n}\right) \\
& =\left(\mathcal{U}_{\Lambda^{n}(R)}-M\right)\left(S_{n}\right)+\left\langle S_{n}, M \omega^{k-s}\right\rangle \\
& \geq c\left(\mathcal{U}_{\Lambda^{n}(R)}-M\right)\left(T_{s}^{+}\right)+\left\langle S_{n}, M \omega^{k-s}\right\rangle \\
& \geq c \mathcal{U}_{T_{s}^{+}, 0}\left(\Lambda^{n}(R)\right)+\left\langle S_{n}-c T_{s}^{+}, M \omega^{k-s}\right\rangle \\
& \geq c \mathcal{U}_{T_{s}^{+}, 0}\left(\Lambda^{n}(R)\right)+M(1-c) .
\end{aligned}
$$

The last term multiplied by $d^{-n}$ goes to zero by Corollary 3.2.10. So the superpotential $U_{L^{n}\left(S_{n}\right)}$ converges to a super-potential of $T_{s}^{+}$on smooth forms. By Proposition A.1.7, that implies that $S=T_{s}^{+}$.

The results of this section remain valid for $f^{-1}$. So we can construct the Green current of order $k-s$ for $f^{-1}$ that we denote by $T_{k-s}^{-}$.

\subsection{Proof of the genericity (Theorem 3.1.11)}

This section is devoted to the proof of Theorem 3.1.11, it is independent from the rest of the paper. The statement of Theorem 3.1.11 is similar to Proposition 4.5 in [1]. In addition, we show here that the genericity stands in any orbit. The idea of the proof is to construct in any orbit an element in $\mathcal{B}$ and then to show that the series in Definition 3.1.9 vary as a difference of psh functions (dsh) along the orbit.

Construction of an example stable by perturbations. - Take $f \in E_{s}$.

We have that:

$$
I(A \circ f)=I(f)
$$

and

$$
I\left(f^{-1} \circ A^{-1}\right)=A I\left(f^{-1}\right) .
$$

In particular, for $A$ generic, we can assume that $I^{+} \cap I^{-}=\varnothing$. Remark also that the dimension of these indeterminacy sets does not depend on $A$. 
Consider a projective linear subspace $E$ of $\mathbb{P}^{k}$ of dimension $s$ such that $E \cap I(f)=\varnothing$. Let $V$ be a neighborhood of $E$ such that $\bar{V} \cap I(f)=\varnothing$.

Choose the coordinates $\left[z_{0}: \ldots: z_{k}\right]$ in $\mathbb{P}^{k}$ such that $E$ is given by $z_{0}=\cdots=$ $z_{k-s-1}=0$ and so that if $E^{\prime}=\left\{z_{k-s}=\cdots=z_{k}=0\right\}$ then $E^{\prime} \cap I\left(f^{-1}\right)=\varnothing$, $E^{\prime} \cap \bar{V}=\varnothing$ and $E^{\prime} \cap \overline{f(V)}=\varnothing$ (this is always possible). Consider the element $A_{0}$ of $\operatorname{PGL}(k+1, \mathbb{C})$ defined by $A_{0}\left(\left[z_{0}: \ldots: z_{k}\right]\right)=\left[\lambda z_{0}: \ldots: \lambda z_{k-s-1}: z_{k-s}: \ldots: z_{k}\right]$ with $\lambda>0$. We take $\lambda$ small enough so that:

- $A_{0}\left(I\left(f^{-1}\right)\right) \subset V$

- $A_{0}(f(V)) \subset V$.

Consider the element $f_{A_{0}}$ defined by $f_{A_{0}}=A_{0} \circ f$. Now, $I\left(f_{A_{0}}\right)=I(f)$ and $I\left(\left(f_{A_{0}}\right)^{-1}\right)=A_{0}\left(I\left(f^{-1}\right)\right) \subset V$. We have the inclusion:

$$
f_{A_{0}}\left(I\left(\left(f_{A_{0}}\right)^{-1}\right)\right)=f_{A_{0}} A_{0}\left(I\left(f^{-1}\right)\right) \subset\left(A_{0} \circ f\right)(V) \subset V .
$$

An immediate induction gives that $\left(f_{A_{0}}\right)^{n}\left(I\left(\left(f_{A_{0}}\right)^{-1}\right)\right) \subset V$. So the element $f_{A_{0}}$ satisfies the first half of Hypothesis 3.1.10 since

$$
\operatorname{dist}\left(I\left(f_{A_{0}}\right), f_{A_{0}}^{n}\left(I\left(f_{A_{0}}^{-1}\right)\right)\right) \geq \operatorname{dist}(I(f), V)>0 .
$$

For each $n, m \in \mathbb{N}$, the condition $f^{n}\left(I\left(f^{-1}\right)\right) \cap f^{-m}(I(f)) \neq \varnothing$ is algebraic (and not always satisfied by the above), so (7) is satisfied outside a countable union of subvarieties of $\operatorname{PGL}(k+1, \mathbb{C})$. Wherever all these conditions are satisfied, namely wherever (7) holds the dynamical degrees are given by Proposition 3.1.6 so they are constant.

We want to show that we can perturbate that example. Fix $E$ and $\bar{V}$ as above. If $\Phi(f):=A \circ f$ with $A$ close to $A_{0}$, then we still have $I\left(\Phi(f)^{-1}\right)=A I\left(f^{-1}\right) \subset V$ and $A \circ f(V) \subset V$. Thus $\Phi(f)^{n}\left(I\left(\Phi(f)^{-1}\right)\right) \subset V$ and $I(\Phi(f))=I(f)$.

This implies that there exists some $\alpha>0$ such that for every $A$ in a small neighborhood $W_{0}^{\prime}$ of $A_{0}$ and every $n \in \mathbb{N}$, we have:

$$
\operatorname{dist}\left(I(\Phi(f)), \Phi(f)^{n}\left(I\left(\Phi(f)^{-1}\right)\right)\right) \geq \alpha .
$$

Since $\mathscr{B}$ and Hypothesis 3.1.10 are invariant by conjugaison and since taking the inverse is a biholomorphism on $\operatorname{PGL}(k+1, \mathbb{C})$, it is equivalent to prove that the set of linear map $A \in \operatorname{PGL}(k+1, \mathbb{C})$ such that $f \circ A \notin \mathscr{B}$ is pluripolar. By the above, we know that there exists a small open set $W_{0}^{\prime}$ in $\operatorname{PGL}(k+1, \mathbb{C})$ where the first series in the definition of $\mathcal{B}$ converges.

Now we prove the genericity. In what follows, $C$ denotes a constant independent of $n$ that may change from line to line. Let $W:=\operatorname{PGL}(k+1, \mathbb{C})$. It is a Zariski dense open set in the projective space $\widetilde{W}=\mathbb{P}^{l}$ where $l=(k+1)^{2}-1$. Let $c$ denote the homogeneous coordinate on $\widetilde{W}$. When $c \in W$, we write $f_{c}$ instead of $f \circ c$. We can extend this notation for $c \in \widetilde{W}$. Of course, in this case $f_{c}$ is not a birational map. 
Consider the rational map:

$$
\begin{aligned}
\widetilde{F}_{n}: \widetilde{W} \times \mathbb{P}^{k} & \rightarrow \widetilde{W} \times \mathbb{P}^{k} \\
(c, z) & \mapsto\left(c, f_{c}^{n}(z)\right) .
\end{aligned}
$$

Let $\Pi_{i}$ denote the canonical projections of $\widetilde{W} \times \mathbb{P}^{k}$ to its factor for $i=1,2$. In $\widetilde{W} \times \mathbb{P}^{k}$, let $\omega_{i}:=\Pi_{i}^{*}\left(\omega_{F S}\right)$ be the pull-back of the Fubini-Study form by the projection for $i=1,2$. That way, $\omega_{1}+\omega_{2}$ is a Kähler form on $\widetilde{W} \times \mathbb{P}^{k}$.

Action of $\widetilde{F}_{n}^{*}$ on the cohomology. - We study the action of $\widetilde{F}_{n}^{*}$ on $\omega_{2}$. Write $c=$ $\left[c_{1}: \ldots: c_{l+1}\right]$. First we have $\widetilde{F}_{n}(c, z)=\left(c, f_{c}^{n}(z)\right)$ where the second coordinate is a polynomial of degree $d^{n}$ in the $z_{i}$, of degree $\leq C d^{n}$ in the $c_{i}$. We compute the mass of $\widetilde{F}_{n}^{*}\left(\omega_{2}\right)$. For that, we test against $\left(\omega_{1}+\omega_{2}\right)^{k+l-1}$. We expand $\left(\omega_{1}+\omega_{2}\right)^{k+l-1}$ :

$$
\left(\omega_{1}+\omega_{2}\right)^{k+l-1}=\sum_{i=0}^{k+l-1}\left(\begin{array}{c}
k+l-1 \\
i
\end{array}\right) \omega_{1}^{i} \wedge \omega_{2}^{k+l-1-i} .
$$

We have that $\omega_{1}^{i}=0$ for $i>l$ and $\omega_{2}^{k+l-1-i}=0$ for $k+l-1-i>k$ thus $i<l-1$. So there are only two terms in the previous sum: for $i=l-1$ and for $i=l$ :

$$
\left\langle\widetilde{F}_{n}^{*}\left(\omega_{2}\right), \omega_{1}^{l-1} \wedge \omega_{2}^{k}\right\rangle \text { and }\left\langle\widetilde{F}_{n}^{*}\left(\omega_{2}\right), \omega_{1}^{l} \wedge \omega_{2}^{k-1}\right\rangle
$$

By Bézout's theorem, those two terms are $\leq C d^{n}$ (the terms can be computed in cohomology so we replace their factors by analytic sets). Here, we use that $\widetilde{F}_{n}(c, z)$ is a polynomial of degree $d^{n}$ in the $z_{i}$ and of degree $\leq C d^{n}$ in the $c_{i}$.

As a result, we have that:

$$
\left\|\widetilde{F}_{n}^{*}\left(\omega_{2}\right)\right\| \leq C d^{n}
$$

and consequently:

$$
\left\|\widetilde{F}_{n}^{*}\left(\omega_{2}^{s}\right)\right\| \leq C d^{s n}
$$

We also remark that:

$$
\widetilde{F}_{n}^{*}\left(\omega_{1}\right)=\omega_{1}
$$

since $\widetilde{F}_{n}$ acts as the identity on $\widetilde{W}$.

Recall that a map in $\mathscr{B}$ needs to satisfies the condition:

$$
\sum_{n=0}^{\infty}\left(\frac{1}{d^{s}}\right)^{n}\left(\operatorname{vol}\left(I^{-}\right)\right)^{-1} \int_{f^{n}\left(I^{-}\right)} U_{L(\omega)} L\left(\omega^{s-1}\right)>-\infty
$$


Construction of a function $g$ that test the convergence of (14). - We can write $\widetilde{F}_{1}^{*}\left(\omega_{2}^{s}\right)$ in cohomology:

$$
\tilde{F}_{1}^{*}\left(\omega_{2}^{s}\right)=\sum_{i_{1}+i_{2}=s} a_{i_{1}, i_{2}} \omega_{1}^{i_{1}} \wedge \omega_{2}^{i_{2}}+d d^{c} \mathcal{U}
$$

where $U$ is a negative $(s-1, s-1)$ current, which is $C^{1}$ where $\widetilde{F}_{1}^{*}\left(\omega_{2}^{s}\right)$ is smooth (see Proposition 2.3.1 in [31] and observe that $\widetilde{W} \times \mathbb{P}^{k}$ is homogeneous). We also denote $\widetilde{\Omega}=\sum_{i_{1}+i_{2}=s} a_{i_{1}, i_{2}} \omega_{1}^{i_{1}} \wedge \omega_{2}^{i_{2}}$ the smooth form cohomologous to $\widetilde{F}_{1}^{*}\left(\omega_{2}^{s}\right)$. Testing against $\omega_{1}^{a} \wedge \omega_{2}^{b}$ for $a+b=l+k-s$ gives that $a_{i_{1}, i_{2}} \geq 0$. In what follows, we take a particular $\mathcal{u}$. We explain now its construction.

Lemma 3.3.1. - The indeterminacy set of $\widetilde{F}_{1}$ has dimension $l+k-s-1$.

Proof. - The lemma is clear in $W \times \mathbb{P}^{k}$. In $(\widetilde{W} \backslash W) \times \mathbb{P}^{k}$, we use a stratification with the dimension of the kernel of $c$.

Indeed, let $F_{j}$ be the set of $A \in \widetilde{W}$ such that $\operatorname{dim}(\operatorname{Ker}(A))=j$ in $\mathbb{C}^{k+1}$. Then, $F_{j}$ is an analytic set of dimension $(k+1)^{2}-j^{2}-1$ (choose $k+1-j$ independant vectors in $\mathbb{C}^{k+1}$ then choose $j$ vectors colinear to the first ones and then project to $\left.\mathbb{P}^{l}\right)$. The indeterminacy set of $\widetilde{F}_{1}$ is given by $\operatorname{Ker}(A) \cup A^{-1}(I(f))$.

Now, $\operatorname{dim}\left(A^{-1}(I(f))\right) \leq k-s-1+j$ so $\operatorname{dim}\left(\operatorname{Ker}(A) \cup A^{-1}(I(f))\right) \leq k-s-1+j$ and the dimension of the indeterminacy set of $\widetilde{F}_{1}$ restricted to $F_{j}$ is of dimension $\leq(k+1)^{2}-j^{2}-1+k-s-1+j \leq(k+1)^{2}-1+k-s-1=l+k-s-1$.

In particular, by Theorem 4.5 in [14, Chapter III], we have that

$$
\widetilde{F}_{1}^{*}\left(\omega_{2}^{s}\right)=\left(\widetilde{F}_{1}^{*}\left(\omega_{2}\right)\right)^{s} .
$$

Since $\widetilde{W} \times \mathbb{P}^{k}$ is a product of projective spaces, then the positive form $\widetilde{F}_{1}^{*}\left(\omega_{2}\right)$ is cohomologous to a positive form $\beta$. Let $u$ be a quasi-potential of $\widetilde{F}_{1}^{*}\left(\omega_{2}\right)$. In other words, $\widetilde{F}_{1}^{*}\left(\omega_{2}\right)=\beta+d d^{c} u$. We can write $\mathcal{U}$ as in the proof of Theorem 3.2 .1 , that is:

$$
\mathcal{U}=\sum_{j=0}^{s-1} u \widetilde{F}_{1}^{*}\left(\omega_{2}\right)^{s-1-j} \wedge \beta^{j}
$$

In this case $\widetilde{\Omega}=\beta^{s}$.

We define $g^{-}:=\widetilde{W} \times I^{-}$. It is an analytic set of $\widetilde{W} \times \mathbb{P}^{k}$ of dimension $l+s-1$ such that for $c \in W, \mathscr{I}^{-} \cap\{c\} \times \mathbb{P}^{k}=I^{-}\left(f_{c}\right)=I^{-}$.

Let $\left[\mathscr{J}^{-}\right]$denote the current of integration on $\mathscr{I}^{-}$, it is of bidimension $(l+s-1, l+$ $s-1)$. Consider the set

$$
V_{n}:=\left\{c \in \widetilde{W}, f_{c}^{-n}\left(I^{+}\left(f_{c}\right)\right) \cap I^{-}=\varnothing\right\},
$$

it is a Zariski dense open set in $\widetilde{W}$.

LEMMA 3.3.2. - The codimension of $V_{n}^{c}=\widetilde{W} \backslash V_{n}$ is $\geq 2$. 
Proof. - Let $E^{\prime} \subset E$ be two linear projective subspaces of $\mathbb{P}^{k}$ with $\operatorname{dim}\left(E^{\prime}\right)=s-1$ and $\operatorname{dim}(E)=s$. Assume that $E \cap I^{+}(f)=\varnothing$. Choose some projective coordinates $\left[z_{0}: \ldots: z_{k}\right]$ such that:

$$
E^{\prime}=\left\{z_{s}=\cdots=z_{k}=0\right\}, E=\left\{z_{s+1}=\cdots=z_{k}=0\right\} .
$$

We denote:

$$
F^{\prime}=\left\{z_{0}=\cdots=z_{s-1}=0\right\}, F=\left\{z_{0}=\cdots=z_{s}=0\right\} .
$$

In particular, $\operatorname{dim}\left(F^{\prime}\right)=k-s$ and $\operatorname{dim}(F)=k-s-1$. Changing the coordinate if necessary, we can assume that $F \cap f(E)=\varnothing$ and $F^{\prime} \cap f\left(E^{\prime}\right)=\varnothing$ and $I^{-} \cap F^{\prime}=\varnothing$ (thus $I^{-} \cap F=\varnothing$ ). Now we consider the following elements of $\widetilde{W}$ :

$$
\begin{aligned}
A^{\prime} & :=\left[z_{0}: \ldots: z_{k}\right] \mapsto\left[z_{0}: \ldots: z_{s-1}: 0: \ldots: 0\right] \\
A & :=\left[z_{0}: \ldots: z_{k}\right] \mapsto\left[z_{0}: \ldots: z_{s}: 0: \ldots: 0\right] .
\end{aligned}
$$

Then observe that $f \circ A\left(\mathbb{P}^{k} \backslash F\right)=f(E)$ and that $f \circ A$ is holomorphic on $f(E)$ with $f \circ A(f(E)) \subset f(E)$; in particular $I\left((f \circ A)^{n}\right)=F$. Similarly $f \circ A^{\prime}\left(\mathbb{P}^{k} \backslash F^{\prime}\right)=f\left(E^{\prime}\right)$, $f \circ A^{\prime}$ is holomorphic on $f\left(E^{\prime}\right)$ with $f \circ A^{\prime}\left(f\left(E^{\prime}\right)\right) \subset f\left(E^{\prime}\right)$; in particular $I\left(\left(f \circ A^{\prime}\right)^{n}\right)=$ $F^{\prime}$. We deduce that $A, A^{\prime} \notin V_{n}^{c}$.

Now consider the compactification $\mathrm{PGL(E, \mathbb {C } )} \subset \widetilde{W}$ where the inclusion is given by the map $B \mapsto B \circ A$ (with obvious notations). Then $\mathrm{PGL(E, \mathbb {C } )}$ is an analytic set of $\widetilde{W}$ of dimension $(s+1)^{2}-1$. First, if $B \in \operatorname{PGL}(E, \mathbb{C})$ then $f \circ B \circ A$ has the same indeterminacy set than $f \circ A$ and the same image, so $B \circ A \notin V_{n}^{c}$. Now consider $\mathrm{PGL}(E, \mathbb{C}) \backslash \operatorname{PGL}(E, \mathbb{C})$, this set is an algebraic irreducible set of codimension 1. Its intersection with $V_{n}^{c}$ is not everything since $A^{\prime} \notin V_{n}^{c}$. In particular, $V_{n}^{c} \cap \widehat{\operatorname{PGL}(E, \mathbb{C})}$ is an analytic set of codimension $\geq 2$. This implies that $V_{n}^{c}$ is not of codimension 1 .

Now, consider on $\cap_{i=0}^{n+1} V_{i}$ :

$$
\varphi_{n}:=\left(\Pi_{1}\right)_{*}\left(\sum_{j=0}^{s-1} \widetilde{F}_{n}^{*} u \widetilde{F}_{n+1}^{*}\left(\omega_{2}\right)^{s-1-j} \wedge \widetilde{F}_{n}^{*}(\beta)^{j} \wedge\left[\mathcal{I}^{-}\right]\right) .
$$

In other words, $\varphi_{n}=\left(\Pi_{1}\right)_{*}\left(\widetilde{F}_{n}^{*}(\mathcal{U}) \wedge\left[\mathscr{J}^{-}\right]\right)$.

On the set $\cap_{i=0}^{n+1} V_{i}, \varphi_{n}$ is continuous since $\Pi_{1}$ restricted to $\mathcal{J}^{-}$is a submersion so the push-forward of a continuous form is continuous. Finally, we define on $\cap_{n=0}^{N+1} V_{n}$,

$$
g_{N}:=\sum_{n=0}^{N} d^{-s n} \varphi_{n}
$$


Computation of $d d^{c} g_{N}$. - First, we compute $d d^{c} g_{N}$ on $\cap_{n=0}^{N+1} V_{n}$. We have that:

$$
d d^{c} g_{N}=\left(\Pi_{1}\right)_{*}\left(\sum_{n=0}^{N} d^{-s n} \sum_{j=0}^{s-1} d d^{c} \widetilde{F}_{n}^{*} u \wedge \widetilde{F}_{n+1}^{*}\left(\omega_{2}\right)^{s-1-j} \wedge \widetilde{F}_{n}^{*}(\beta)^{j} \wedge\left[\mathcal{I}^{-}\right]\right) \text {. }
$$

Recall that $d d^{c} u=\widetilde{F}_{1}^{*}\left(\omega_{2}\right)-\beta$. So, near $\mathscr{I}^{-}$

$$
d d^{c} \widetilde{F}_{n}^{*} u=\widetilde{F}_{n+1}^{*}\left(\omega_{2}\right)-\widetilde{F}_{n}^{*}(\beta) .
$$

We obtain

$$
\begin{aligned}
& d d^{c} g_{N}= \\
& \left(\Pi_{1}\right)_{*}\left(\sum_{n=0}^{N} d^{-s n} \sum_{j=0}^{s-1}\left(\widetilde{F}_{n+1}^{*}\left(\omega_{2}\right)^{s-j} \wedge \widetilde{F}_{n}^{*}(\beta)^{j}-\widetilde{F}_{n+1}^{*}\left(\omega_{2}\right)^{s-1-j} \wedge \widetilde{F}_{n}^{*}(\beta)^{j+1}\right) \wedge\left[\mathscr{J}^{-}\right]\right)
\end{aligned}
$$

which is equal to

$$
d d^{c} g_{N}=\left(\Pi_{1}\right)_{*}\left(\sum_{n=0}^{N} d^{-s n}\left(\widetilde{F}_{n+1}^{*}\left(\omega_{2}^{s}\right)-\widetilde{F}_{n}^{*}\left(\beta^{s}\right)\right) \wedge\left[\mathscr{J}^{-}\right]\right) .
$$

Recall that $\beta^{s}=\widetilde{\Omega}=\sum_{i_{1}+i_{2}=s} a_{i_{1}, i_{2}} \omega_{1}^{i_{1}} \wedge \omega_{2}^{i_{2}}$. We show now that $a_{0, s}=d^{s}$. First we have that:

$$
\left\langle\widetilde{\Omega}, \omega_{2}^{k-s} \wedge \omega_{1}^{l}\right\rangle=a_{0, s} .
$$

Since $\widetilde{\Omega}$ and $\widetilde{F}_{1}^{*}\left(\omega_{2}^{s}\right)$ are cohomologous, we deduce:

$$
\left\langle\widetilde{F}_{1}^{*}\left(\omega_{2}^{s}\right), \omega_{2}^{k-s} \wedge \omega_{1}^{l}\right\rangle=a_{0, s} .
$$

So, we want to compute:

$$
\int \widetilde{F}_{1}^{*}\left(\omega_{2}^{s}\right) \wedge \omega_{2}^{k-s} \wedge \omega_{1}^{l}
$$

This can be done in cohomology. If $L_{k-s}$ is a generic analytic subspace of dimension $k-s$ in $\mathbb{P}^{k}$ and $L_{s}$ is a generic analytic subspace of dimension $s$ in $\mathbb{P}^{k}$ and $\{c\} \times \mathbb{P}^{k}$ is a slice, then the previous quantity is the number of intersections of $f_{c}^{-1}\left(L_{k-s}\right) \cap L_{s}$ on the slice. This is equal to $d^{s}$ since the degree $d_{s}$ of $f_{c}$ is $d^{s}$ on $W$ which is a Zariski open set in $\widetilde{W}$, so we have indeed that $d_{s}=a_{0, s}$.

We have the equality:

$$
\widetilde{F}_{n}^{*}(\widetilde{\Omega})=d^{s} \widetilde{F}_{n}^{*}\left(\omega_{2}^{s}\right)+\sum_{i_{1}+i_{2}=s, i_{2} \neq s} a_{i_{1}, i_{2}} \widetilde{F}_{n}^{*}\left(\omega_{1}^{i_{1}} \wedge \omega_{2}^{i_{2}}\right) .
$$

We denote the second term on the right-hand side by $S_{n}$. Since $\widetilde{F}_{n}^{*}\left(\omega_{1}\right)=\omega_{1}$, we can bound the mass of $S_{n}$ by:

$$
\begin{aligned}
\left\|S_{n}\right\| & \leq \sum_{i_{1}+i_{2}=s, i_{2} \neq s} a_{i_{1}, i_{2}}\left\|\omega_{1}^{i_{1}} \wedge \widetilde{F}_{n}^{*}\left(\omega_{2}^{i_{2}}\right)\right\| \\
& \leq C d^{n(s-1)}
\end{aligned}
$$


since $\left\|\widetilde{F}_{n}^{*}\left(\omega_{2}^{j}\right)\right\| \leq C d^{j n}$ for $j \leq s$. So replacing in $d d^{c} g_{N}$, we have:

$$
\begin{aligned}
d d^{c} g_{N}= & \left(\Pi_{1}\right)_{*}\left(\sum_{n=0}^{N} d^{-s n}\left(\widetilde{F}_{n+1}^{*}\left(\omega_{2}^{s}\right)-d^{s} \widetilde{F}_{n}^{*}\left(\omega_{2}^{s}\right)\right) \wedge\left[\mathcal{I}^{-}\right]\right) \\
& -\left(\Pi_{1}\right)_{*}\left(\sum_{n=0}^{N} d^{-s n} S_{n} \wedge\left[\mathcal{I}^{-}\right]\right) .
\end{aligned}
$$

The second term in the right-hand side is a positive closed current with mass uniformly bounded in $N$ by the above. We now control the first term. Reorganizing the sum, we see that it is equal to:

$$
\left(\Pi_{1}\right)_{*}\left(\left(d^{-s N} \widetilde{F}_{N+1}^{*}\left(\omega_{2}^{s}\right)-d^{s} \omega_{2}^{s}\right) \wedge\left[\mathscr{J}^{-}\right]\right) .
$$

Using the fact that the mass of the positive closed current $\widetilde{F}_{N+1}^{*}\left(\omega_{2}^{s}\right)$ is bounded by $C d^{s(N+1)}$ gives that, on $\cap_{n=0}^{N+1} V_{n}$ :

$$
d d^{c} g_{N}=\Omega_{1, N}^{+}-\Omega_{2, N}^{+}
$$

where $\Omega_{i, N}^{+}$is a positive closed current of bidegree $(1,1)$ on $\cap_{n=0}^{N+1} V_{n}$ with mass less than a constant $C(C$ is independent of $N)$. So, the current $\Omega_{i, N}^{+}$has an extension to a positive closed current $\widetilde{\Omega_{i, N}^{+}}$with $\left\|\widetilde{\Omega_{i, N}^{+}}\right\| \leq C$. Now, we prove that the function $g_{N}$ is in $L^{1}(\widetilde{W})$ and that $d d^{c} g_{N}=\widetilde{\Omega_{1, N}^{+}}-\widetilde{\Omega_{2, N}^{+}}$in the sense of currents of $\widetilde{W}$. Take an open set $U$ in $\widetilde{W}$ biholomorphic to a polydisk. On $U$, we have $\widetilde{\Omega_{i, N}^{+}}=d d^{c} u_{i, N}$ where $u_{i, N}$ is a negative psh function. So, on $U \cap\left(\cap_{n=0}^{N+1} V_{n}\right)$,

$$
g_{N}+u_{2, N}=u_{1, N}+h_{N}
$$

with $h_{N}$ a harmonic function. The equality stands almost everywhere but since $g_{N}$ is continuous on $\cap_{n=0}^{N+1} V_{n}$, the equality is true on $U \cap\left(\cap_{n=0}^{N+1} V_{n}\right)$ (see the proof of the inequality $g-m \geq \psi_{1}-\psi_{2}$ below). Since $g_{N}$ is non positive, the function $u_{1, N}+h_{N}$ has a psh extension $u_{1, N}+h_{N}$ to $U$. So $g_{N}$ has an extension $\widetilde{g_{N}}=\widetilde{u_{1, N}+h_{N}}-u_{2, N}$ to $U$. In particular, $g_{N}$ is in $L^{1}(\widetilde{W})$ and $d d^{c} g_{N}$ has a sense globally. Moreover, since the codimension of $V_{n}^{c}$ is larger than 2, we have on $U$ (in the sense of currents):

$$
d d^{c} g_{N}=d d^{c} \widetilde{g_{N}}=d d^{c} u_{1, N}+h_{N}-d d^{c} u_{2, N}=\widetilde{\Omega_{1, N}^{+}}-\widetilde{\Omega_{2, N}^{+}} .
$$

In particular, we have $d d^{c} g_{N}=\widetilde{\Omega_{1, N}^{+}}-\widetilde{\Omega_{2, N}^{+}}$globally on $\widetilde{W}$.

Now, we write

$$
\widetilde{\Omega_{i, N}^{+}}=a_{i, N} \omega_{1}+d d^{c} \psi_{i, N}
$$

with $a_{i, N}$ smaller than $C$.

We say that a measure is $P L B$ if the qpsh functions are integrable for that measure. Any measure given by a smooth distribution is $P L B$. In particular, we can find a $P L B$ 
probability measure that we denote $\nu$ with support in the $W_{0}^{\prime}$ defined previously. We have the following lemma (see Proposition 2.4 in [27]):

LEMMA 3.3.3. - The family of qpsh functions in $\widetilde{W}$ such that $d d^{c} \psi \geq-\omega_{1}$ and one of the two following conditions:

$$
\max _{\widetilde{W}} \psi=0 \text { or } \int \psi d \nu=0
$$

is bounded in $L^{1}(\nu)$ and is bounded from above.

In particular, in the writing

$$
\widetilde{\Omega_{i, N}^{+}}=a_{i, N} \omega_{1}+d d^{c} \psi_{i, N}
$$

we take the normalization $\int \psi_{i, N} d \nu=0$.

Link with Definition 3.1.9. - Let $c \in W \cap\left(\cap_{i \leq n+1} V_{i}\right)$, then we want to show that:

$$
\varphi_{n}(c)=\int_{f_{c}^{n}\left(I^{-}\left(f_{c}\right)\right)} U_{c}
$$

where $\left(f_{c}\right)^{*}\left(\omega_{2}^{s}\right)=d^{s} \omega_{2}^{s}+d d^{c} U_{c}$. First, when $c \in W \cap\left(\cap_{i \leq n+1} V_{i}\right)$, we have:

$$
\varphi_{n}(c)=\int_{I^{-}\left(f_{c}\right)} \widetilde{F}_{n}^{*}(\mathcal{U})_{\mid\{c\} \times \mathbb{P}^{k}} .
$$

Here, $\widetilde{F}_{n}^{*}(\mathcal{U})_{\mid\{c\} \times \mathbb{P}^{k}}$ is the restriction of $\widetilde{F}_{n}^{*}(\mathcal{U})$ on $\{c\} \times \mathbb{P}^{k}$ which is well defined because $\widetilde{F}_{n}^{*}(\mathcal{U})$ is continuous near $\{c\} \times I^{-}\left(f_{c}\right)$. But $\widetilde{F}_{n}^{*}(\mathcal{U})_{\mid\{c\} \times \mathbb{P}^{k}}$ is equal to $\left(f_{c}^{n}\right)^{*}\left(\mathcal{U}_{\mid\{c\} \times \mathbb{P}^{k}}\right)$, so

Recall that

$$
\varphi_{n}(c)=\int_{f_{c}^{n}\left(I^{-}\left(f_{c}\right)\right)} \mathcal{U}_{\mid\{c\} \times \mathbb{P}^{k}}
$$

$$
\mathcal{U}=\sum_{j=0}^{s-1} u \widetilde{F}_{1}^{*}\left(\omega_{2}\right)^{s-1-j} \wedge \beta^{j}
$$

In particular, $\mathcal{U}_{\mid\{c\} \times \mathbb{P}^{k}}$ near $f_{c}^{n}\left(I^{-}\left(f_{c}\right)\right)$ can be written

$$
U_{c}:=\sum_{j=0}^{s-1} u_{\mid\{c\} \times \mathbb{P}^{k}} f_{c}^{*}\left(\omega_{2}\right)^{s-1-j} \wedge d^{j} \omega_{2}^{j}
$$

because the coefficient of $\beta$ in $\omega_{2}$ is $d\left(\beta=d \omega_{2}+\ldots\right)$. We show now that $d d^{c} U_{c}=$ $\left(f_{c}\right)^{*}\left(\omega_{2}^{s}\right)-d^{s} \omega_{2}^{s}$.

The singularities of $u_{\mid\{c\} \times \mathbb{P}^{k}}$ are in $I^{+}\left(f_{c}\right)$, so by Theorem 4.5 in [14, Chapter III], we have that $U_{c}$ and $d d^{c} U_{c}=\sum_{j=0}^{s-1} d d^{c}\left(u_{\mid\{c\} \times \mathbb{P}^{k}}\right) \wedge f_{c}^{*}\left(\omega_{2}\right)^{s-1-j} \wedge d^{j} \omega_{2}^{j}$ are well defined in all $\mathbb{P}^{k}$. Taking the restriction of the equation $\widetilde{F}_{1}^{*}\left(\omega_{2}\right)=\beta+d d^{c} u$ on $\{c\} \times \mathbb{P}^{k}$, we obtain

$$
f_{c}^{*}\left(\omega_{2}\right)=d \omega_{2}+\left(d d^{c} u\right)_{\mid\{c\} \times \mathbb{P}^{k}}=d \omega_{2}+d d^{c}\left(u_{\mid\{c\} \times \mathbb{P}^{k}}\right)
$$


since it is true outside $I^{+}\left(f_{c}\right)$ and $f_{c}^{*}\left(\omega_{2}\right)$ or $d d^{c}\left(u_{\mid\{c\} \times \mathbb{P}^{k}}\right)$ have no mass on this set of dimension $k-s-1$. Moreover $u$ is a qpsh function, so it takes a value at every point.

Finally,

$$
d d^{c} U_{c}=\sum_{j=0}^{s-1}\left(f_{c}^{*}\left(\omega_{2}\right)-d \omega_{2}\right) \wedge f_{c}^{*}\left(\omega_{2}\right)^{s-1-j} \wedge d^{j} \omega_{2}^{j}=\left(f_{c}\right)^{*}\left(\omega_{2}^{s}\right)-d^{s} \omega_{2}^{s} .
$$

Proof of the genericity. - Recall that $\varphi_{n}$ is continuous on $\cap_{i \leq n+1} V_{i}$. It implies that $g_{N}$ is continuous on $\cap_{i \leq N+1} V_{i}$ and it decreases to a function $g$ on $\cap_{i \geq 0} V_{i}$ with $g$ usc on $\cap_{i \geq 0} V_{i}$. It means that for every point $x$ in $\Lambda=\cap_{i \geq 0} V_{i}$, we have $\lim \sup _{y \rightarrow x, y \in \Lambda} g(y) \leq$ $g(x)$.

Let $m_{N}=\int g_{N} d \nu$. We can write on $\widetilde{W}, g_{N}-m_{N}=\psi_{1, N}-\psi_{2, N}$. Here the equality is true on a set of full Lebesgue measure in $\widetilde{W}$. But, since $g_{N}$ is continuous on $\cap_{i \leq N+1} V_{i}$ and the $\psi_{i, N}$ are qpsh, the equality is true for every point in $\cap_{i \leq N+1} V_{i}$ (see below the proof of the inequality $g-m \geq \psi_{1}-\psi_{2}$ ).

We apply the previous lemma to the sequences $\psi_{i, N}$ and we have that these sequences are uniformly bounded from above and bounded in $L^{1}(\nu)$. So we can extract converging subsequences to some limit points $\psi_{1}$ and $\psi_{2}$ in $L^{1}$. The sequence $m_{N}$ is bounded thanks to the definitions of $W_{0}^{\prime}$ and $\nu$. So $m_{N}$ converges to $m$ by monotone convergence. In particular, $g-m=\psi_{1}-\psi_{2}$ up to a set of zero Lebesgue measure in $\widetilde{W}$. We want to show now that we have

$$
g-m \geq \psi_{1}-\psi_{2}
$$

for every point in $\Lambda=\cap_{i \geq 0} V_{i}$. Indeed, assume there is a point $x \in \Lambda$ such that $\left(g+\psi_{2}\right)(x)<m+\psi_{1}(x)-\varepsilon$. On a chart which contains $x$, we can write $\psi_{1}=\lambda_{1}+\xi_{1}$ with $\lambda_{1}$ smooth and $\xi_{1}$ psh.

Since $g$ and $\psi_{2}$ are usc on $\Lambda$, so is their sum and so $\left(g+\psi_{2}\right)(y)<m+\psi_{1}(x)-\varepsilon / 2$ on a small ball $B(x, r)$ centered at $x$ and of radius $r$ (for $y \in \Lambda$ ). For a function $h$, we denote by $m_{B(x, r)}(h)$ the mean value of $h$ on the ball $B(x, r)$. We have that that $m_{B(x, r)}\left(g+\psi_{2}\right)=m_{B(x, r)}\left(m+\lambda_{1}+\xi_{1}\right)$ since both functions are equal a.e. and $m_{B(x, r)}\left(\xi_{1}\right) \geq \xi_{1}(x)$ since $\xi_{1}$ is psh, so

$$
m+\psi_{1}(x)-\varepsilon / 2 \geq m_{B(x, r)}\left(g+\psi_{2}\right) \geq m_{B(x, r)}\left(\lambda_{1}\right)+m+\xi_{1}(x)
$$

which is false if we take $r$ small enough to have $m_{B(x, r)}\left(\lambda_{1}\right)$ near $\lambda_{1}(x)$.

In particular, the set of points where $g=-\infty$ is pluripolar since it is included in the set of points where $\psi_{1}$ is $-\infty$. By the proof of Theorem 3.2.1, we see that $g \neq-\infty$ is equivalent to the fact that the first half of Definition 3.1.9 is satisfied.

We do the same thing for the second half of Definition 3.1.9 and we conclude since the intersection of two pluripolar sets is pluripolar. 


\subsection{The equilibrium measure}

3.4.1. Construction of the measure. - We want to define the equilibrium measure $\mu$ as $T_{s}^{+} \wedge T_{k-s}^{-}$. In [1], the authors used an approach based on the energy. More precisely, they show that the potential of the Green current is in the Hilbert space $H_{T^{-}}$defined by the closure of the smooth forms for the norm $\sqrt{\int d \varphi \wedge d^{c} \varphi \wedge T^{-}}$. They deduced from that fact that the measure $T^{+} \wedge T^{-}$is well defined and that the potential of the Green current is integrable with respect to that measure.

That approach cannot be adapted here since the super-potential is not a function defined on $\mathbb{P}^{k}$ but a function defined on $\mathscr{C}_{k-s+1}$. Instead, we will use the formalism of super-potential. See Definition A.1.13 for the definition of wedgeability. We prove the theorem:

THEOREM 3.4.1. - The current $T_{s}^{+}$and $T_{k-s}^{-}$are wedgeable. So the intersection $T_{s}^{+} \wedge$ $T_{k-s}^{-}$is a well defined probability measure $\mu$ and the quasi-potential of the Green current of order 1 is integrable with respect to this measure.

Recall that $T^{+}:=T_{1}^{+}$is a well defined invariant current in $\mathscr{C}_{1}([\mathbf{5 0}])$ and that it admits the quasi-potential:

$$
G:=\sum_{n}\left(\frac{1}{d} f^{*}\right)^{n} u
$$

where $u<0$ is a quasi-potential of the current $d^{-1} f^{*}(\omega)$ (we write $u$ instead of $U_{L(\omega)}$ in order to simplify the notations). We denote as before $L_{n}$ and $\Lambda_{n}$ the normalized pull-pack and push-forward associated to $f^{n}$. In what follows, for $q \leq s, \mathcal{U}_{L^{m}\left(\omega^{q}\right)}$ denotes the super-potential of $L^{m}\left(\omega^{q}\right)$ given by (8) in Lemma 3.2.2, that is:

$$
u_{L^{m}\left(\omega^{q}\right)}=\sum_{n=0}^{m-1} \frac{1}{d^{n}} u_{L\left(\omega^{q}\right)} \circ \Lambda^{n},
$$

on $\left(f^{m}\right)_{*}$-admissible currents in $\mathscr{C}_{k-q+1}$.

We need the following lemma to construct the measure.

LEMmA 3.4.2. - The current $\omega^{s-q} \wedge L^{n}\left(\omega^{q}\right)$ and $T_{k-s}^{-}$are wedgeable for all $n \geq 0$ and $0 \leq q \leq s$. Furthermore, for all integers $n$ and $n^{\prime}$ and $1 \leq q \leq s+1$ we have that $\mathcal{U}_{L^{n}(\omega)^{q}}\left(L^{n^{\prime}}(\omega)^{s-q+1} \wedge T_{k-s}^{-}\right)$is finite.

Proof. - We have seen in Proposition 3.2.11 that $\omega^{s-q} \wedge L(\omega)^{q}$ and $T_{k-s}^{-}$are wedgeable for $q \leq s$ and that the super-potentials of $T_{k-s}^{-}$are finite at $L(\omega)^{s+1}$. So applying that to $f^{n}$ instead of $f$, we have that $\omega^{s-q} \wedge L^{n}(\omega)^{q}$ and $T_{k-s}^{-}$are wedgeable for all $n \geq 0$ and that the super-potentials of $T_{k-s}^{-}$are finite at $L^{n}(\omega)^{s+1}$.

The case where $q=s+1$ is already known so we assume $1 \leq q \leq s$. The current $L(\omega)^{s+1-q}$ and $L(\omega)^{q}$ are wedgeable and their wedge-product is $L(\omega)^{s+1}$ (it follows 
from Corollary 4.11 Chapter III in [14] and Lemma A.1.16). So using Lemma A.2.1 we have that a super-potential of $L(\omega)^{s+1}$ is given by:

$$
\mathcal{U}_{L(\omega)^{q}}\left(L(\omega)^{s-q+1} \wedge R\right)+\mathcal{U}_{L(\omega)^{s-q+1}}\left(\omega^{q} \wedge R\right)
$$

on current $R \in \mathscr{C}_{k-s}$ such that $R$ and $L(\omega)^{s-q+1}$ are wedgeable. In particular, we can take $R=T_{k-s}^{-}$at which point the super-potential of $L(\omega)^{s+1}$ is finite. A superpotential of $L(\omega)^{s-q+1} \wedge \omega^{q}$ is given by:

$$
U_{L(\omega)^{s-q+1}}\left(\omega^{q} \wedge \star\right) .
$$

So by difference,

$$
\mathcal{U}_{L(\omega)^{q}}\left(L(\omega)^{s-q+1} \wedge T_{k-s}^{-}\right)
$$

is well defined in the sense of super-potentials (that is it is continuous for the Hartogs' convergence) and is finite.

So we have proved the lemma for $n=n^{\prime}=1$.

Applying the result to $f^{n}$ gives the lemma for $n=n^{\prime}$. Now, let $n \leq n^{\prime}$. Then $L^{n}(\omega)^{q}$ is more H-regular than $L^{n^{\prime}}(\omega)^{q}$. The super-potentials of $L^{n^{\prime}}(\omega)^{q}$ are finite at $L^{n^{\prime}}(\omega)^{s-q+1} \wedge T_{k-s}^{-}$so the super-potentials of $L^{n}(\omega)^{q}$ are also finite at $L^{n^{\prime}}(\omega)^{s-q+1} \wedge$ $T_{k-s}^{-}$.

Similarly, let $n \geq n^{\prime}$. Then $L^{n^{\prime}}(\omega)^{s-q+1}$ is more H-regular than $L^{n}(\omega)^{s-q+1}$ and so Lemma A.1.14 implies that $L^{n^{\prime}}(\omega)^{s-q+1} \wedge T_{k-s}^{-}$is more H-regular than $L^{n}(\omega)^{s-q+1} \wedge$ $T_{k-s}^{-}$. The super-potentials of $L^{n}(\omega)^{q}$ are finite at $L^{n}(\omega)^{s-q+1} \wedge T_{k-s}^{-}$, which means by symmetry of the super-potentials that the super-potentials of $L^{n}(\omega)^{s-q+1} \wedge T_{k-s}^{-}$are finite at $L^{n}(\omega)^{q}$. Hence the super-potentials of $L^{n^{\prime}}(\omega)^{s-q+1} \wedge T_{k-s}^{-}$are finite at $L^{n}(\omega)^{q}$ which means that the super-potentials of $L^{n}(\omega)^{q}$ are finite at $L^{n^{\prime}}(\omega)^{s-q+1} \wedge T_{k-s}^{-}$. That gives the lemma.

Proof of Theorem 3.4.1. - By the above lemma, we have that $L^{n}\left(\omega^{s}\right) \wedge T_{k-s}^{-}$is $\left(f^{n}\right)_{*}$-admissible since it is finite at $U_{L^{n}(\omega)}$. Hence by Lemma 3.1 .8 , we have that $\Lambda^{n}\left(L^{n}\left(\omega^{s}\right) \wedge T_{k-s}^{-}\right)$is well defined and equal to $\Lambda_{n}\left(L_{n}\left(\omega^{s}\right) \wedge T_{k-s}^{-}\right)$(recall that Corollary 3.1.7 gives $\left.L_{n}\left(\omega^{s}\right)=L^{n}\left(\omega^{s}\right)\right)$.

We consider:

$$
\frac{1}{d^{n}} \mathcal{U}_{L(\omega)}\left(\Lambda_{n}\left(L_{n}\left(\omega^{s}\right) \wedge T_{k-s}^{-}\right)\right) .
$$

It is finite since by Lemma A.2.2 applied to $f^{n}$ and the invariance ot $T_{k-s}^{-}$, it is equal to

$$
\frac{1}{d^{n}} \mathcal{U}_{L(\omega)}\left(\omega^{s} \wedge T_{k-s}^{-}\right)
$$

and the previous lemma assures us that this is finite. 
We use Lemma A.2.3 for $f^{n}$ instead of $f$ with $S_{1}=L(\omega), S_{2}=\omega^{s}$ and $S_{3}=T_{k-s}^{-}$. So we see that the previous quantity is equal to:

$$
\mathcal{U}_{L^{n}\left(\omega^{s}\right)}\left(L^{n+1}(\omega) \wedge T_{k-s}^{-}\right)-\mathcal{U}_{L^{n}\left(\omega^{s}\right)}\left(L^{n}(\omega) \wedge T_{k-s}^{-}\right)+\left(\frac{1}{d}\right)^{n} \mathcal{U}_{L(\omega)}\left(\Lambda_{n}\left(\omega^{s} \wedge T_{k-s}^{-}\right)\right) .
$$

We now perform some sort of Abel transform. We sum from 0 to $N$ and we regroup the terms in $L^{n}(\omega) \wedge T_{k-s}^{-}$(observe for the first term that $\left.U_{\omega^{s}}=0\right)$ :

$$
\begin{aligned}
\sum_{n=0}^{N} \frac{1}{d^{n}} u_{L(\omega)}\left(\omega^{s} \wedge T_{k-s}^{-}\right) & =\sum_{n=1}^{N}\left(-\mathcal{U}_{L^{n}\left(\omega^{s}\right)}+\mathcal{U}_{L^{n-1}\left(\omega^{s}\right)}\right)\left(L^{n}(\omega) \wedge T_{k-s}^{-}\right) \\
& +\mathcal{U}_{L^{N}\left(\omega^{s}\right)}\left(L^{N+1}(\omega) \wedge T_{k-s}^{-}\right)+\sum_{n=0}^{N} \frac{1}{d^{n}} \mathcal{U}_{L(\omega)}\left(\Lambda_{n}\left(\omega^{s} \wedge T_{k-s}^{-}\right)\right)
\end{aligned}
$$

Now, $U_{L^{n-1}\left(\omega^{s}\right)}-U_{L^{n}\left(\omega^{s}\right)}=-d^{-n+1} U_{L\left(\omega^{s}\right)} \circ \Lambda^{n-1}$ on $\left(f^{n}\right)_{*}$-admissible currents by Lemma 3.2.2. In particular, we consider the current $L^{n}(\omega) \wedge T_{k-s}^{-}$which is $\left(f^{n}\right)_{*^{-}}$ admissible by the previous lemma. So using again Lemma A.2.2 for $f^{n-1}$ gives:

$$
\begin{aligned}
\left(\mathcal{U}_{L^{n-1}\left(\omega^{s}\right)}-\mathcal{U}_{L^{n}\left(\omega^{s}\right)}\right)\left(L^{n}(\omega) \wedge T_{k-s}^{-}\right) & =-d^{-n+1} u_{L\left(\omega^{s}\right)}\left(\Lambda_{n-1}\left(L^{n}(\omega) \wedge T_{k-s}^{-}\right)\right) \\
& =-d^{-n+1} u_{L\left(\omega^{s}\right)}\left(\Lambda_{n-1}\left(L_{n-1}(L(\omega)) \wedge T_{k-s}^{-}\right)\right) \\
& =-d^{-n+1} u_{L\left(\omega^{s}\right)}\left(L(\omega) \wedge T_{k-s}^{-}\right) .
\end{aligned}
$$

So the series $\sum_{n=1}^{N}\left(-\mathcal{U}_{L^{n}\left(\omega^{s}\right)}+\mathcal{U}_{L^{n-1}\left(\omega^{s}\right)}\right)\left(L^{n}(\omega) \wedge T_{k-s}^{-}\right)$is also convergent thanks to the previous lemma. We also have that $\mathcal{U}_{L^{N}\left(\omega^{s}\right)}\left(L^{N+1}(\omega) \wedge T_{k-s}^{-}\right)$is negative since $\mathcal{U}_{L^{N}\left(\omega^{s}\right)}$ is negative. Thus, letting $N$ go to $\infty$ :

$$
\begin{array}{r}
\left(\sum_{n \geq 0} \frac{1}{d^{n}}\right) \mathcal{U}_{L(\omega)}\left(\omega^{s} \wedge T_{k-s}^{-}\right)+\left(\sum_{n \geq 1} d^{-n+1}\right) \mathcal{u}_{L\left(\omega^{s}\right)}\left(L(\omega) \wedge T_{k-s}^{-}\right) \\
\leq \sum_{n \geq 0} \frac{1}{d^{n}} \mathcal{U}_{L(\omega)}\left(\Lambda^{n}\left(\omega^{s} \wedge T_{k-s}^{-}\right)\right) .
\end{array}
$$

We recognize by (8) that the right-hand side is in fact $\mathcal{U}_{T^{+}}\left(\omega^{s} \wedge T_{k-s}^{-}\right)$which in term of quasi-potential is $\int G \omega^{s} \wedge T_{k-s}^{-}$(recall that $T^{+}$is the Green current of order 1). Thus by Hartogs' convergence, we have that $\mathcal{U}_{T^{+}}\left(\omega^{s} \wedge T_{k-s}^{-}\right)$is finite (we could also conclude by monotone convergence that $G \in L^{1}\left(\omega^{s} \wedge T_{k-s}^{-}\right)$, both properties being equivalent).

Observe now that in (16) every term converge. In particular,

$$
\left(\mathcal{U}_{L^{N}\left(\omega^{s}\right)}\left(L^{N+1}(\omega) \wedge T_{k-s}^{-}\right)\right)_{N}
$$


converges to a finite value. Using Lemma A.2.4, we have the identity:

$$
\begin{aligned}
U_{L^{N}\left(\omega^{s}\right)}\left(L^{N+1}(\omega) \wedge T_{k-s}^{-}\right) & =U_{L^{N+1}(\omega)}\left(L^{N}\left(\omega^{s}\right) \wedge T_{k-s}^{-}\right) \\
& -U_{L^{N+1}(\omega)}\left(\omega^{s} \wedge T_{k-s}^{-}\right)+U_{L^{N}\left(\omega^{s}\right)}\left(\omega \wedge T_{k-s}^{-}\right) .
\end{aligned}
$$

On the right-hand side, the first and third terms are negative, the third term is decreasing and we just proved that the second term converges to $U_{T^{+}}\left(\omega^{s} \wedge T_{k-s}^{-}\right)$ which is finite. That implies that every term is in fact convergent.

In particular, we have the convergence of $\mathcal{U}_{L^{N}\left(\omega^{s}\right)}\left(\omega \wedge T_{k-s}^{-}\right)$. Since $L^{N}\left(\omega^{s}\right) \rightarrow T_{s}^{+}$ in the Hartogs' sense, that means that $\mathcal{U}_{T_{s}^{+}}\left(\omega \wedge T_{k-s}^{-}\right)$is finite. Hence the current $T_{s}^{+}$ and $T_{k-s}^{-}$are wedgeable and their intersection is a well defined probability measure $\mu$ (we could also have deduced that from the convergence of the first term but this is more natural).

Now, recall that the function $(R, S) \rightarrow \mathcal{U}(R, S):=\mathcal{U}_{R}(S)=\mathcal{U}_{S}(R)$ for $R$ and $S$ in $\mathscr{C}_{q}$ and $\mathscr{C}_{k-q}\left(\mathcal{U}_{R}\right.$ and $\mathcal{U}_{S}$ being the super-potentials of mean 0$)$ is upper semicontinuous. The convergence of $\mathcal{U}\left(L^{N+1}(\omega), L^{N}\left(\omega^{s}\right) \wedge T_{k-s}^{-}\right)$implies that $\mathcal{U}\left(T^{+}, T_{s}^{+} \wedge\right.$ $\left.T_{k-s}^{-}\right)$is finite which means exactly that the quasi-potential of the Green current is integrable with respect to $\mu$.

Of course, the measure $\mu$ also integrate the potential of the Green current of order 1 of $f^{-1}$.

Corollary 3.4.3. - The measure $\mu$ gives no mass to the indeterminacy sets $I^{+}$and $I^{-}$. Furthermore, $L(\mu)=f^{*}(\mu)$ and $\Lambda(\mu)=f_{*}(\mu)$ are well defined in the sense of super-potentials.

Proof. - The fact that $\mu$ is $f_{*}$-admissible follows from Theorem 3.4 .1 since its superpotentials are finite at the point $L\left(T^{+}\right)=T^{+}$and so they are finite at the point $L(\omega)$ which is more H-regular than $L\left(T^{+}\right)$. Since the potential of $T^{+}$is equal to $-\infty$ on $I^{+}$ and is in $L^{1}(\mu)$ (in fact $\log \operatorname{dist}\left(x, I^{+}\right) \in L^{1}(\mu)$ ) we have that $\mu$ gives no mass to the indeterminacy set $I^{+}$, similarly for $I^{-}$.

Proposition 3.4.4. - The measure $\mu$ is invariant, that is $f^{*}(\mu)$ and $f_{*}(\mu)$ are equal to $\mu$.

Proof. - The currents $L^{n}\left(\omega^{s}\right)$ and $\Lambda^{m}\left(\omega^{k-s}\right)$ are wedgeable for $m$ and $n$ in $\mathbb{N}$ since they are more H-regular than $T_{s}^{+}$and $T_{k-s}^{-}$. So let $\mu_{n}:=L^{n}\left(\omega^{s}\right) \wedge \Lambda^{n}\left(\omega^{k-s}\right)$ (resp. $\left.\mu_{n}^{\prime}:=L^{n-1}\left(\omega^{s}\right) \wedge \Lambda^{n+1}\left(\omega^{k-s}\right)\right)$. Now since $L^{n}\left(\omega^{s}\right)$ and $\Lambda^{n}\left(\omega^{k-s}\right)$ converge in the Hartogs' sense to $T_{s}^{+}$and $T_{k-s}^{-}$which are wedgeable, we have that $\mu_{n}$ (resp. $\mu_{n}^{\prime}$ ) converges to $\mu$ in Hartogs' sense (Proposition A.1.15).

By Lemma A.2.2, we have that $\mu_{n}^{\prime}=\Lambda\left(L^{n}\left(\omega^{s}\right) \wedge \Lambda^{n}\left(\omega^{k-s}\right)\right)=\Lambda\left(\mu_{n}\right)$ (observe that $L^{n}\left(\omega^{s}\right) \wedge \Lambda^{n}\left(\omega^{k-s}\right)$ is $f_{*}$ admissible since it is more H-regular than $T_{s}^{+} \wedge T_{k-s}^{-}$which is $f_{*}$-admissible). 
So, since $\mu$ is $f_{*}$-admissible, we have that $\mu_{n}^{\prime}$ converges in the Hartogs' sense to $\Lambda(\mu)=\mu$ which is what we wanted.

Corollary 3.4.5. - The measure $\mu$ gives no mass to the indeterminacy sets $I\left(f^{ \pm n}\right)$ and the critical sets $\mathscr{C}\left(f^{ \pm n}\right)$.

Proof. - We already know that the indeterminacy sets have no mass for $\mu$ so using the invariance of $\mu$, we have that $\mu(\mathscr{C}(f))=\mu\left(f^{-1}\left(I^{-}\right)\right)=\mu\left(I^{-}\right)=0$.

3.4.2. Green currents of order $1 \leq q \leq s$. - The purpose of this paragraph is to construct the Green currents of order $q$ for $q \leq s$. This will allow us to prove that $T_{s}^{+}$ can be written as $\left(T^{+}\right)^{s}$. As an application, we show that the equilibrium measure gives no mass to the pluripolar sets.

Using the same arguments than in Theorem 3.4.1, we construct the Green currents $T_{q}^{+}$of order $q$ for $q \leq s$ :

Proposition 3.4.6. - For $1 \leq q \leq s$, the sequence $\left(L^{n}\left(\omega^{q}\right)\right)_{n}$ converges in the Hartogs' sense to $T_{q}^{+}$the Green current of order $q$ and the Green currents $T_{q}^{+}$and $T_{k-s}^{-}$ are wedgeable. Furthermore, any super-potential $\mathcal{U}_{T_{q}^{+}}$of $T_{q}^{+}$satisfies

$$
\mathcal{U}_{T_{q}^{+}}\left(T_{s-q+1}^{+} \wedge T_{k-s}^{-}\right)>-\infty .
$$

Proof. - Observe that the roles of $q$ and $s-q+1$ are symmetric, so anything proved for $q$ stands for $s-q+1$. The current $L^{n}\left(\omega^{s-q+1}\right) \wedge T_{k-s}^{-}$is $\left(f^{n}\right)_{*}$ admissible by Lemma 3.4.2. Lemma 3.1.8 implies that $\Lambda^{n}\left(L^{n}\left(\omega^{s-q+1}\right) \wedge T_{k-s}^{-}\right)$is well defined and equal to $\Lambda_{n}\left(L^{n}\left(\omega^{s-q+1}\right) \wedge T_{k-s}^{-}\right)$. So we consider this time:

$$
\frac{1}{d^{n}} \mathcal{U}_{L\left(\omega^{q}\right)}\left(\Lambda_{n}\left(L^{n}\left(\omega^{s-q+1}\right) \wedge T_{k-s}^{-}\right)\right) .
$$

By Lemma A.2.2 and the invariance ot $T_{k-s}^{-}$, it is equal to

$$
\frac{1}{d^{n}} U_{L\left(\omega^{q}\right)}\left(\omega^{s-q+1} \wedge T_{k-s}^{-}\right)
$$

and Lemma 3.4.2 assures us that this is finite.

Using Lemma A.2.3, performing the same Abel transform and using again Lemma A.2.2, we obtain similarly that:

$$
\begin{aligned}
\left(\sum_{n=0}^{N} \frac{1}{d^{n}}\right) U_{L\left(\omega^{q}\right)}\left(\omega^{s-q+1} \wedge T_{k-s}^{-}\right) & =\left(\sum_{n=1}^{N}-d^{-n+1}\right) \mathcal{U}_{L\left(\omega^{s-q+1}\right)}\left(L\left(\omega^{q}\right) \wedge T_{k-s}^{-}\right) \\
& +\mathcal{U}_{L^{N}\left(\omega^{s-q+1}\right)}\left(L^{N+1}\left(\omega^{q}\right) \wedge T_{k-s}^{-}\right) \\
& +\sum_{n=0}^{N} \frac{1}{d^{n}} \mathcal{U}_{L\left(\omega^{q}\right)}\left(\Lambda^{n}\left(\omega^{s-q+1} \wedge T_{k-s}^{-}\right)\right)
\end{aligned}
$$


We have again that $\mathcal{U}_{L^{N}\left(\omega^{s-q+1}\right)}\left(L^{N+1}\left(\omega^{q}\right) \wedge T_{k-s}^{-}\right)$is negative since $\mathcal{U}_{L^{N}\left(\omega^{s-q+1}\right)}$ is negative. Thus, letting $N$ go to $\infty$ :

$$
\begin{array}{r}
\left(\sum_{n \geq 0} d^{-n}\right) \mathcal{U}_{L\left(\omega^{q}\right)}\left(\omega^{s-q+1} \wedge T_{k-s}^{-}\right)+\left(\sum_{n \geq 1} d^{-n+1}\right) \mathcal{U}_{L\left(\omega^{s-q+1}\right)}\left(L\left(\omega^{q}\right) \wedge T_{k-s}^{-}\right) \\
\leq \sum_{n \geq 0} \frac{1}{d^{n}} \mathcal{U}_{L\left(\omega^{q}\right)}\left(\Lambda_{n}\left(\omega^{s-q+1} \wedge T_{k-s}^{-}\right)\right) .
\end{array}
$$

Again, by Proposition A.1.8, we have that the sequence of super-potential of $L^{n}\left(\omega^{q}\right)$ is decreasing thus to have the convergence in the Hartogs' sense, it is sufficient to have the convergence at one point. We recognize by (8) that the right-hand side gives in fact the convergence at the point $\omega^{s-q+1} \wedge T_{k-s}^{-}$(again $\omega^{s-q+1} \wedge T_{k-s}^{-}$is $\left(f^{n}\right)_{*}$-admissible so $\left.\Lambda_{n}\left(\omega^{s-q+1} \wedge T_{k-s}^{-}\right)=\Lambda^{n}\left(\omega^{s-q+1} \wedge T_{k-s}^{-}\right)\right)$. So we have that $\mathcal{U}_{T_{q}^{+}}\left(\omega^{s-q+1} \wedge T_{k-s}^{-}\right)$ is finite and $L^{n}\left(\omega^{q}\right)$ converges to $T_{q}^{+}$in the Hartogs' sense.

In (17) every term converges. In particular,

$$
\left(\mathcal{U}_{L^{N}\left(\omega^{s-q+1}\right)}\left(L^{N+1}\left(\omega^{q}\right) \wedge T_{k-s}^{-}\right)\right)_{N}
$$

converges to a finite value. Using Lemma A.2.4, we have the identity:

$$
\begin{aligned}
\mathcal{U}_{L^{N}\left(\omega^{s-q+1}\right)}\left(L^{N+1}\left(\omega^{q}\right) \wedge T_{k-s}^{-}\right) & =U_{L^{N+1}\left(\omega^{q}\right)}\left(L^{N}\left(\omega^{s-q+1}\right) \wedge T_{k-s}^{-}\right) \\
& -U_{L^{N+1}\left(\omega^{q}\right)}\left(\omega^{s-q+1} \wedge T_{k-s}^{-}\right) \\
& +U_{L^{N}\left(\omega^{s-q+1}\right)}\left(\omega^{q} \wedge T_{k-s}^{-}\right) .
\end{aligned}
$$

As above, every term converges to a finite value. In particular, that means that $U_{T_{s-q+1}^{+}}\left(\omega^{q} \wedge T_{k-s}^{-}\right)$is finite (which is already known by exchanging the role of $q$ and $s-q+1)$. Finally the convergence of $\mathcal{U}_{L^{N+1}\left(\omega^{q}\right)}\left(L^{N}\left(\omega^{s-q+1}\right) \wedge T_{k-s}^{-}\right)$implies that $\mathcal{U}\left(T_{q}^{+}, T_{s-q+1}^{+} \wedge T_{k-s}^{-}\right)$is finite.

We prove that $T_{q}^{+}$is invariant.

LEMMA 3.4.7. - For $1 \leq q \leq s$, the Green current $T_{q}^{+}$is $f^{*}$-admissible and satisfies $T_{q}^{+}=L\left(T_{q}^{+}\right)$. Furthermore, $T_{q}^{+}$is the most $H$-regular current which is $f^{*}$-invariant in $\mathscr{C}_{q}$. In particular, $T_{q}^{+}$is extremal in the set of $f^{*}$-invariant currents of $\mathscr{C}_{q}$.

Proof. - For $q=s$, this is Theorem 3.2.9. So take $q<s$. We have that $L^{n}\left(\omega^{q}\right)$ converges in the Hartogs' sense to $T_{q}^{+}$. So this means that at the point $\omega^{k-q+1}$ we have the convergence of the series:

$$
\sum_{n \geq 0} d^{-n} u_{L\left(\omega^{q}\right)}\left(\Lambda^{n}\left(\omega^{k-q+1}\right)\right) .
$$


In particular, dropping the first term and multiplying by $d$, we have the convergence of the series:

$$
\sum_{n \geq 0} d^{-n} \mathcal{U}_{L\left(\omega^{q}\right)}\left(\Lambda^{n}\left(\Lambda\left(\omega^{k-q+1}\right)\right)\right) .
$$

We recognize $\mathcal{U}_{T_{q}^{+}}\left(\Lambda\left(\omega^{k-q+1}\right)\right)>-\infty$ hence $T_{q}^{+}$is $f^{*}$-admissible.

By Theorem A.1.17, we see that $L\left(L^{n}\left(\omega^{q}\right)\right)$ converges to $L\left(T_{q}^{+}\right)$and to $T_{q}^{+}$. So we have proved the first part of the lemma. The rest is exactly as in Theorem 3.2.9.

Now, we also want to consider the intersection $T_{q}^{+} \wedge T_{k-s}^{-}$for $q<s$. First, we have that these intersections are well defined elements of $\mathscr{C}_{k-s+q}$ from Proposition 3.4.6 $\left(T_{q}^{+}\right.$and $T_{k-s}^{-}$are wedgeable). Furthermore, it is $f_{*}$-admissible since we have by Proposition 3.4.6 that

$$
\mathcal{U}_{T_{s-q+1}^{+}}\left(T_{q}^{+} \wedge T_{k-s}^{-}\right)>-\infty
$$

and since $L\left(\omega^{s-q+1}\right)$ is more H-regular than $T_{s-q+1}^{+}=L\left(T_{s-q+1}^{+}\right)$, we see that:

$$
\mathcal{U}_{L\left(\omega^{s-q+1}\right)}\left(T_{q}^{+} \wedge T_{k-s}^{-}\right)>-\infty,
$$

which means that $T_{q}^{+} \wedge T_{k-s}^{-}$is $f_{*}$-admissible since by symmetry of the super-potential, its super-potentials are finite at the point $L\left(\omega^{s-q+1}\right)$.

Using the same argument than in the proof of the invariance of the measure $\mu$, one has:

Proposition 3.4.8. - The current $T_{q}^{+} \wedge T_{k-s}^{-} \in \mathscr{C}_{k-s+q}$ is $f_{*}$-invariant, that is:

$$
\Lambda\left(T_{q}^{+} \wedge T_{k-s}^{-}\right)=T_{q}^{+} \wedge T_{k-s}^{-} .
$$

Proof. - This follows from the fact that $L^{n}\left(\omega^{q}\right)$ and $\Lambda^{m}\left(\omega^{k-s}\right)$ converge in the Hartogs' sense to $T_{q}^{+}$and $T_{k-s}^{-}$which are wedgeable and we use Proposition A.2.2.

Now, we use the same arguments than in the proof of Theorem 3.4.1, but we replace $T_{k-s}^{-}$by $T_{q}^{+} \wedge T_{k-s}^{-}$. Our purpose is to show that $T_{s}^{+}=\left(T^{+}\right)^{s}$. We need the following lemma first:

LEMMA 3.4.9. - Let $q_{1} \geq 1$ and $q_{2}$ such that $q_{1}+q_{2}=s-q+1$. Then the current $T_{q_{2}}^{+}$ and $T_{q}^{+} \wedge T_{k-s}^{-}$are wedgeable and we have that a super-potential $\mathcal{U}_{T_{q_{1}}^{+}}$of $T_{q_{1}}^{+}$satisfies:

$$
\mathcal{U}_{T_{q_{1}}^{+}}\left(T_{q_{2}}^{+} \wedge T_{q}^{+} \wedge T_{k-s}^{-}\right)>-\infty .
$$

The proof is essentially the same as the one of Theorem 3.4.1. We need the equivalent of Lemma 3.4.2 first:

LEMMA 3.4.10. - Let $q_{1} \geq 1$ and $q_{2}$ such that $q_{1}+q_{2}=s-q+1$ and let $n \in \mathbb{N}$. Then the currents $L^{n}\left(\omega^{q_{2}}\right)$ and $T_{q}^{+} \wedge T_{k-s}^{-}$are wedgeable. Furthermore, for $n^{\prime} \in \mathbb{N}$ :

$$
u_{L^{n^{\prime}}\left(\omega^{q_{1}}\right)}\left(L^{n}\left(\omega^{q_{2}}\right) \wedge T_{q}^{+} \wedge T_{k-s}^{-}\right)>-\infty .
$$


Proof. - We can assume that $q_{2} \geq 1$ (else it is just Proposition 3.4.6). The superpotentials of the current $T_{q}^{+} \wedge T_{k-s}^{-}$are finite at $L\left(\omega^{q_{1}+q_{2}}\right)=L\left(\omega^{q_{1}}\right) \wedge L\left(\omega^{q_{2}}\right)$ which is less H-regular than $\omega^{q_{1}} \wedge L\left(\omega^{q_{2}}\right)$. Hence the super-potentials of the current $T_{q}^{+} \wedge T_{k-s}^{-}$ are finite at $\omega^{q_{1}} \wedge L\left(\omega^{q_{2}}\right)$. This means that the currents $L\left(\omega^{q_{2}}\right)$ and $T_{q}^{+} \wedge T_{k-s}^{-}$are wedgeable.

On the other hand, $\mathcal{U}_{L\left(\omega^{\left.q_{1}+q_{2}\right)}\right.}\left(T_{q}^{+} \wedge T_{k-s}^{-}\right)$is finite. We can use Lemma A.2.1 and we have that:

$U_{L\left(\omega^{\left.q_{1}+q_{2}\right)}\right.}\left(T_{q}^{+} \wedge T_{k-s}^{-}\right)=\mathcal{U}_{L\left(\omega^{q_{2}}\right)}\left(\omega^{q_{1}} \wedge T_{q}^{+} \wedge T_{k-s}^{-}\right)+\mathcal{U}_{L\left(\omega^{q_{1}}\right)}\left(L\left(\omega^{q_{2}}\right) \wedge T_{q}^{+} \wedge T_{k-s}^{-}\right)$.

Again taking the difference with $\mathcal{U}_{L\left(\omega^{\left.q_{2}\right)}\right.}\left(\omega^{q_{1}} \wedge T_{q}^{+} \wedge T_{k-s}^{-}\right)$, we have that:

$$
U_{L\left(\omega^{q_{1}}\right)}\left(L\left(\omega^{q_{2}}\right) \wedge T_{q}^{+} \wedge T_{k-s}^{-}\right)
$$

is well defined in the sense of super-potentials and is finite. We have proved the lemma for $n=n^{\prime}=1$. The rest follows as in Lemma 3.4.2.

Proof of Lemma 3.4.9. - We replace $T_{k-s}^{-}$by $T_{q}^{+} \wedge T_{k-s}^{-}$and we do the same computations. Lemma A.2.2, A.2.3 and A.2.4 still apply for $T_{q}^{+} \wedge T_{k-s}^{-}$.

We can now prove the following corollary. Observe that if a sequence $S_{n}$ converges in the Hartogs' sense to $S$ and a sequence $R_{n}$ converges in the Hartogs' sense to $R$ with $R_{n} \wedge S_{n}$ wedgeable converging in the Hartogs' sense to a current $T$, we cannot claim a priori that $S$ and $R$ are wedgeable and that $T=R \wedge S$. But if $S$ and $R$ are wedgeable, then we do have $T=R \wedge S$.

Corollary 3.4.11. - The current $T_{s}^{+}$satisfies $T_{s}^{+}=\left(T^{+}\right)^{s}$. Consequently, one has $\mu=\left(T^{+}\right)^{s} \wedge\left(T^{-}\right)^{k-s}$ where $T^{ \pm}$is the Green current of order 1 of $f^{ \pm}$.

Proof. - Applying the previous lemma to $q=1, q_{1}=1$ and $q_{2}=s-1$ gives that

$$
\mathcal{U}_{T^{+}}\left(T_{s-1}^{+} \wedge T^{+} \wedge T_{k-s}^{-}\right)>-\infty \text {. }
$$

Since $\omega^{k-s+1}$ is more H-regular than $T^{+} \wedge T_{k-s}^{-}$that implies that:

$$
U_{T^{+}}\left(T_{s-1}^{+} \wedge \omega^{k-s+1}\right)>-\infty .
$$

In particular that $T^{+}$and $T_{s-1}^{+}$are wedgeable. Since $L^{n}(\omega)$ and $L^{n}\left(\omega^{s-1}\right)$ converges in the Hartogs' sense to $T^{+}$and $T_{s-1}^{+}$and $L^{n}\left(\omega^{s}\right)$ converges in the Hartogs' sense to $T_{s}^{+}$, Proposition A.1.15 implies that $T^{+} \wedge T_{s-1}^{+}=T_{s}^{+}$. An easy induction gives the result for $T_{s}^{+}$and the result follows for $\mu$.

REMARK 3.4.12. - We do not know how to prove the previous result without constructing $T_{k-s}^{-}$first. In the case where $f$ satisfies Hypothesis 3.1.10, the result was proved directly (see Theorem 3.2.8). This illustrates the difference between Hypotheses 3.1.10 and Definition 3.1.9. For a map satisfying Hypotheses 3.1.10, we have that 
the potential of $T^{+}$is finite at every point of $I^{-}$, if it is only in $\mathcal{B}$, we can only say that $\int_{I^{-}} U_{T^{+}} \omega^{s-1}$ is finite since $T^{+} \wedge \omega^{s-1}$ is more H-regular than $T_{s}^{+}=\left(T^{+}\right)^{s}$.

Now, we improve the previous results and we show that the measure $\mu$ gives no mass to pluripolar sets (hence analytic sets). The proof relies on a space of test functions introduced by Dinh and Sibony in [26] and studied by the second author in [53]. Recall that the space $W^{1,2}\left(\mathbb{P}^{k}\right)$ is the set of functions in $L^{2}$ whose differential in the sense of currents can be represented by a form in $L^{2}$. The space $W^{*}\left(\mathbb{P}^{k}\right)$ is the set of functions $\varphi$ in $W^{1,2}\left(\mathbb{P}^{k}\right)$ such that there exists a positive closed current $S_{\varphi}$ of bidegree $(1,1)$ satisfying:

$$
d \varphi \wedge d^{c} \varphi \leq S_{\varphi}
$$

For $\varphi \in W^{*}$, we define the norm:

$$
\|\varphi\|_{*}^{2}=\|\varphi\|_{L^{2}}^{2}+\inf \{m(S), S \text { closed, satisfying }(18)\} .
$$

Let $\psi$ be a qpsh function in $W^{*}\left(\mathbb{P}^{k}\right)$. Consider the regularization $\psi_{n}$ of $\psi$ obtained through an approximation of the identity in $\operatorname{PGL}(k+1, \mathbb{C})$. Let $S$ be minimal in (18) for $\psi$ and let $S_{n}$ be the smooth regularization of $S$ obtained through the same approximation of the identity. Using Lemma 5 in [53], we have

- $\psi_{n}$ "decreases" to $\psi$.

$-d \psi_{n} \wedge d^{c} \psi_{n} \leq S_{n}$, and $m\left(S_{n}\right) \rightarrow m(S)$ thus $\lim \left\|\psi_{n}\right\|_{*}=\|\psi\|_{*}$.

If $\varphi$ is a qpsh function in $\mathbb{P}^{k}$ with $\varphi \leq-2$, then $\psi:=-\log -\varphi$ is in $W^{*}\left(\mathbb{P}^{k}\right)$, thus for every pluripolar set in $\mathbb{P}^{k}$ there exists a qpsh function in $W^{*}\left(\mathbb{P}^{k}\right)$ equal to $-\infty$ on that set (see Example 1 p. 253 in [53]). In particular, if the qpsh functions in $W^{*}\left(\mathbb{P}^{k}\right)$ are integrable for a measure, the measure cannot give mass to the pluripolar sets. We can now state the theorem:

THEOREM 3.4.13. - The measure $\mu$ gives no mass to pluripolar sets (hence analytic sets). More precisely, there exists $C>0$ such that for $\psi<0$ a qpsh function in $W^{*}\left(\mathbb{P}^{k}\right)$, we have that:

$$
|\mu(\psi)| \leq C\|\psi\|_{*} .
$$

Proof. - Let $\psi$ and $\psi_{n}$ be as above. Recall that $G$ is the potential of $T^{+}$. Let $T_{m}^{+}$ and $T_{m}^{-}$be sequence of smooth currents in $\mathscr{C}_{1}$ converging to $T^{+}$and $T^{-}$in the Hartogs' sense. Then $\mu_{m}=\left(T_{m}^{+}\right)^{s} \wedge\left(T_{m}^{-}\right)^{k-s}$ converges to $\mu$ in the Hartogs' sense by Proposition A.1.15. Let $G_{m}$ be the associated potential of $T_{m}^{+}$. Using Stokes' formula 
and Cauchy-Schwarz inequality, we have that:

$$
\begin{aligned}
\left|\int \psi_{n} d \mu_{m}\right| & =\left|\int \psi_{n}\left(d d^{c} G_{m}+\omega\right) \wedge\left(T_{m}^{+}\right)^{s-1} \wedge\left(T_{m}^{-}\right)^{k-s}\right| \\
& \leq\left|\int d \psi_{n} \wedge d^{c} G_{m} \wedge\left(T_{m}^{+}\right)^{s-1} \wedge\left(T_{m}^{-}\right)^{k-s}\right| \\
& +\left|\int \psi_{n} \omega \wedge\left(T_{m}^{+}\right)^{s-1} \wedge\left(T_{m}^{-}\right)^{k-s}\right| \\
& \leq\left(\int d \psi_{n} \wedge d^{c} \psi_{n} \wedge\left(T_{m}^{+}\right)^{s-1} \wedge\left(T_{m}^{-}\right)^{k-s}\right)^{\frac{1}{2}} \\
& \times\left(\int d G_{m} \wedge d^{c} G_{m} \wedge\left(T_{m}^{+}\right)^{s-1} \wedge\left(T_{m}^{-}\right)^{k-s}\right)^{\frac{1}{2}} \\
& +\left|\int \psi_{n} \omega \wedge\left(T_{m}^{+}\right)^{s-1} \wedge\left(T_{m}^{-}\right)^{k-s}\right| .
\end{aligned}
$$

Let $S_{n}$ be the positive closed current of bidegree $(1,1)$ such that $d \psi_{n} \wedge d^{c} \psi_{n} \leq S_{n}$. Using again Stokes' formula for the second term of the product yields:

$$
\begin{aligned}
\left|\int \psi_{n} d \mu_{m}\right| & \leq\left(\int S_{n} \wedge\left(T_{m}^{+}\right)^{s-1} \wedge\left(T_{m}^{-}\right)^{k-s}\right)^{\frac{1}{2}} \\
& \times\left(-\int G_{m} d d^{c} G_{m} \wedge\left(T_{m}^{+}\right)^{s-1} \wedge\left(T_{m}^{-}\right)^{k-s}\right)^{\frac{1}{2}} \\
& +\left|\int \psi_{n} \omega \wedge\left(T_{m}^{+}\right)^{s-1} \wedge\left(T_{m}^{-}\right)^{k-s}\right|
\end{aligned}
$$

We let $m$ go to $\infty$, we have that $\left|\int \psi_{n} d \mu_{m}\right|$ converges to $\left|\int \psi_{n} d \mu\right|$,

$$
\left(\int S_{n} \wedge\left(T_{m}^{+}\right)^{s-1} \wedge\left(T_{m}^{-}\right)^{k-s}\right)
$$

converges to $\left(\int S_{n} \wedge T_{s-1}^{+} \wedge T_{k-s}^{-}\right)$, and $\left|\int \psi_{n} \omega \wedge\left(T_{m}^{+}\right)^{s-1} \wedge\left(T_{m}^{-}\right)^{k-s}\right|$ converges to $\left|\int \psi_{n} \omega \wedge T_{s-1}^{+} \wedge T_{k-s}^{-}\right|$. The term:

$$
\begin{aligned}
\int G_{m} d d^{c} G_{m} \wedge\left(T_{m}^{+}\right)^{s-1} \wedge\left(T_{m}^{-}\right)^{k-s}= & \int G_{m}\left(T_{m}^{+}\right)^{s} \wedge\left(T_{m}^{-}\right)^{k-s} \\
& -\int G_{m} \omega \wedge\left(T_{m}^{+}\right)^{s-1} \wedge\left(T_{m}^{-}\right)^{k-s}
\end{aligned}
$$

can be rewritten as:

$$
\mathcal{U}_{1}\left(T_{m}^{+},\left(T_{m}^{+}\right)^{s} \wedge\left(T_{m}^{-}\right)^{k-s}\right)-\mathcal{U}_{1}\left(T_{m}^{+}, \omega \wedge\left(T_{m}^{+}\right)^{s-1} \wedge\left(T_{m}^{-}\right)^{k-s}\right)
$$

which by Hartogs' convergence goes with $m$ to:

$$
\mathcal{U}_{1}\left(T^{+}, \mu\right)-\mathcal{U}_{1}\left(T^{+}, \omega \wedge T_{s-1}^{+} \wedge T_{k-s}^{-}\right)
$$


which is finite by Theorem 3.4.1 (observe that $\omega \wedge T_{s-1}^{+} \wedge T_{k-s}^{-}$is more H-regular than $\mu)$. So we have that:

$$
\left|\int \psi_{n} d \mu\right| \leq C\left(\int S_{n} \wedge T_{s-1}^{+} \wedge T_{k-s}^{-}\right)^{\frac{1}{2}}+\left|\int \psi_{n} \omega \wedge T_{s-1}^{+} \wedge T_{k-s}^{-}\right|,
$$

where $C^{2}=\left|\mathcal{U}_{1}\left(T^{+}, \mu\right)-\mathcal{U}_{1}\left(T^{+}, \omega \wedge T_{s-1}^{+} \wedge T_{k-s}^{-}\right)\right|$is a constant.

The term $\left(\int S_{n} \wedge T_{s-1}^{+} \wedge T_{k-s}^{-}\right)^{\frac{1}{2}}$ is controlled by $\|\psi\|_{*}+\varepsilon$ for $n$ large enough because $S_{n}$ is smooth so that wedge-product is well defined and the mass can be computed in cohomology.

We use an induction to control in the same way the term $\left|\int \psi_{n} \omega \wedge T_{s-1}^{+} \wedge T_{k-s}^{-}\right|$ (at the last step, we have a term in $\int-\psi_{n} \omega^{k}$ ). Since for $n$ large enough we have $\left\|\psi_{n}\right\|_{*} \leq\|\psi\|_{*}+\varepsilon(\varepsilon>0)$, we have proved that:

$$
\left|\int \psi_{n} d \mu\right| \leq C\left(\|\psi\|_{*}+\varepsilon\right) .
$$

By monotone convergence and letting $\varepsilon \rightarrow 0$, we have the theorem.

3.4.3. Mixing, entropy and hyperbolicity of $\mu$. - We now prove that $\mu$ is mixing, that is $\lim _{n \rightarrow \infty} \mu\left(\varphi \psi \circ f^{n}\right)=\mu(\varphi) \mu(\psi)$ for $\varphi$ and $\psi$ smooth functions on $\mathbb{P}^{k}$. Here the function $\psi \circ f^{n}$ is not smooth, so by definition $\mu\left(\varphi \psi \circ f^{n}\right)$ is the integral of $\varphi \psi \circ f^{n}$ on $\mathbb{P}^{k} \backslash I\left(f^{n}\right)$ for the measure $\mu$ which gives no mass to $I\left(f^{n}\right)$. Recall that $I\left(f^{n}\right) \subset \mathscr{C}\left(f^{n}\right)$.

We need the classical lemma ([50] and $[42])$ :

LEMMA 3.4.14. - Let $\psi$ be smooth function on $\mathbb{P}^{k}$, then the sequence of currents $\left(\psi \circ f^{n} T_{s}^{+}\right)_{n}$ converges to $c T_{s}^{+}$where $c=\mu(\psi)$. Moreover, we have that $\left\|d\left(\psi \circ f^{n} T_{s}^{+}\right)\right\|$ and $\left\|d d^{c}\left(\psi \circ f^{n} T_{s}^{+}\right)\right\|$go to zero.

Proof. - The norm $\left\|d\left(\psi \circ f^{n} T_{s}^{+}\right)\right\|$is the operator's norm on the space of smooth forms.

We can assume that $0 \leq \psi \leq 1$. Then, the sequence $\left(\psi \circ f^{n} T_{s}^{+}\right)_{n}$ is bounded so we can extract a subsequence converging in the sense of currents to $S \geq 0$ which satisfies $S \leq T_{s}^{+}$. In order to show that $S$ is closed and that $\left\|d\left(\psi \circ f^{n} T_{s}^{+}\right)\right\| \rightarrow 0$, we only need to show that for every smooth $(0,1)$-form $\theta$ we have that $\left|\left\langle\psi \circ f^{n} T_{s}^{+}, \partial\left(\theta \wedge \omega^{k-s-1}\right)\right\rangle\right|$ goes to 0 uniformly on $\theta$ (see [12] p. 3 for details). In other words, we want to compute the limit of:

$$
\int_{\mathbb{P}^{k} \backslash I\left(f^{n}\right)} \psi \circ f^{n} T_{s}^{+} \wedge \partial(\theta) \wedge \omega^{k-s-1} .
$$

We are going to use the technics of [49]. Let $v<0$ be a qpsh function equal to $-\infty$ on $\mathscr{C}\left(f^{n}\right)$ and smooth outside $\mathscr{C}\left(f^{n}\right)$. Let $\max ^{\prime}$ be a smooth convex increasing function approximating the function $\max ^{+}:=\max (x, 0)$ such that its derivative is less 
than 1 . Let $v_{j}=\max ^{\prime}(v / j+1)$. Then $\left(v_{j}\right)$ is an increasing sequence of smooth qpsh $\left(i \partial \bar{\partial} v_{j}+\omega \geq 0\right)$ functions with $0 \leq v_{j} \leq 1$ converging uniformly to 1 on the compact sets of $\mathbb{P}^{k} \backslash \mathscr{C}\left(f^{n}\right)$ and equal to 0 on some neighborhood of $\mathscr{C}\left(f^{n}\right)$. Let $\alpha:[0,1] \rightarrow[0,1]$ be a smooth function equal to 0 in $[0,1 / 3]$ and to 1 in $[2 / 3,1]$. Then the sequence of functions $v_{j}^{\prime}:=\alpha \circ v_{j}$ is equal to 1 on the compact sets of $\mathbb{P}^{k} \backslash \mathscr{C}\left(f^{n}\right)$ for $j$ large enough and is equal to 0 on some neighborhood of $\mathscr{C}\left(f^{n}\right)$.

Since $T_{s}^{+}$gives no mass to $\mathscr{C}\left(f^{n}\right)$, the previous quantity is the limit when $j$ goes to $\infty$ of:

$$
\left\langle v_{j}^{\prime} \psi \circ f^{n} T_{s}^{+}, \partial(\theta) \wedge \omega^{k-s-1}\right\rangle .
$$

By Stokes' formula, it is equal to:

$$
-\left\langle v_{j}^{\prime} \partial\left(\psi \circ f^{n}\right) \wedge T_{s}^{+}, \theta \wedge \omega^{k-s-1}\right\rangle-\left\langle\psi \circ f^{n} \partial\left(v_{j}^{\prime}\right) \wedge T_{s}^{+}, \theta \wedge \omega^{k-s-1}\right\rangle .
$$

We apply Cauchy-Schwarz inequality for the first term of the sum, we bound the absolute value of the first term of the previous quantity by:

$$
\left\langle\left(v_{j}^{\prime}\right)^{2} i \partial \psi \circ f^{n} \wedge \bar{\partial} \psi \circ f^{n} \wedge T_{s}^{+}, \omega^{k-s-1}\right\rangle^{\frac{1}{2}} \times\left\langle i \theta \wedge \bar{\theta} \wedge T_{s}^{+}, \omega^{k-s-1}\right\rangle^{\frac{1}{2}} .
$$

The second term of the product is bounded and does not depend on $j$ and $n$ (uniformly in $\|\theta\|)$. For the first term, observe that :

$$
i \partial \psi \circ f^{n} \wedge \bar{\partial} \psi \circ f^{n} \wedge T_{s}^{+}=d^{-s n}\left(f^{n}\right)^{*}\left(i \partial \psi \wedge \bar{\partial} \psi \wedge T_{s}^{+}\right)
$$

in the integral since $f^{n}$ is smooth on the support of $v_{j}^{\prime}$ and one can multiply a positive closed current by a smooth form and take the pull-back by a smooth function. So, assuming that $i \partial \psi \wedge \bar{\partial} \psi \leq \omega$, we have that the first term is less than:

$$
\left\langle d^{-s n}\left(f^{n}\right)^{*}\left(\omega \wedge T_{s}^{+}\right), \omega^{k-s-1}\right\rangle^{\frac{1}{2}}=\left(\delta^{-(k-s) n} \delta^{(k-s-1) n}\right)^{\frac{1}{2}}=\delta^{-n / 2}
$$

which goes to 0 when $n$ goes to $\infty$ independtly of $j$.

Now we have to control the term:

$$
\left\langle\partial\left(v_{j}^{\prime}\right) \psi \circ f^{n} \wedge T_{s}^{+}, \theta \wedge \omega^{k-s-1}\right\rangle .
$$

We have that $\partial\left(v_{j}^{\prime}\right)=\alpha^{\prime}\left(v_{j}\right) \partial v_{j}$ and observe that the sequence of functions $\left(\alpha^{\prime}\left(v_{j}\right)\right)$ is bounded and converges uniformly to 0 on the compact sets of $\mathbb{P}^{k} \backslash \mathscr{C}\left(f^{n}\right)$. We apply Cauchy-Schwarz inequality and we get that:

$$
\begin{array}{r}
\left\langle\partial\left(v_{j}^{\prime}\right) \psi \circ f^{n} \wedge T_{s}^{+}, \theta \wedge \omega^{k-s-1}\right\rangle^{2} \leq \\
\left\langle i \partial\left(v_{j}\right) \wedge \bar{\partial}\left(v_{j}\right) \wedge T_{s}^{+}, \omega^{k-s-1}\right\rangle\left\langle i\left(\alpha^{\prime}\left(v_{j}\right)\right)^{2} \theta \wedge \bar{\theta} \wedge T_{s}^{+}, \omega^{k-s-1}\right\rangle .
\end{array}
$$

The first term of the product is equal by Stokes' formula to:

$$
\left\langle-v_{j} \wedge i \partial \bar{\partial}\left(v_{j}\right) \wedge T_{s}^{+}, \omega^{k-s-1}\right\rangle
$$

Since $0 \leq v_{j} \leq 1$ and $i \partial \bar{\partial} v_{j}+\omega \geq 0$, it is less than:

$$
\left\langle v_{j} \omega \wedge T_{s}^{+}, \omega^{k-s-1}\right\rangle \leq\left\langle\omega \wedge T_{s}^{+}, \omega^{k-s-1}\right\rangle
$$


which is bounded independently of $n$ and $j$. The second term of the product goes to 0 when $j \rightarrow \infty$ uniformly on $\theta$ by dominated convergence since $T_{s}^{+}$gives no mass to $\mathscr{C}\left(f^{n}\right)$. So letting $j \rightarrow \infty$ first, we see that:

$$
\left\langle\psi \circ f^{n} T_{s}^{+}, \partial(\theta) \wedge \omega^{k-s-1}\right\rangle
$$

goes to 0 when $n \rightarrow \infty$ uniformly on $\|\theta\|$.

By Theorem 3.2.19, this shows that $S=c T_{s}^{+}$. To compute $c$, consider $\langle\psi \circ$ $\left.f^{n} T_{s}^{+}, \omega^{k-s}\right\rangle$. It is equal to $\left\langle T_{s}^{+} \wedge \Lambda^{n}\left(\omega^{k-s}\right), \psi\right\rangle$ : replace $T_{s}^{+}$by a smooth approximation $T_{m}^{+}$, then $\psi \circ f^{n} L^{n}\left(T_{m}^{+}\right)=d^{-n s}\left(f^{n}\right)^{*}\left(\psi T_{m}^{+}\right)$, so

$$
\left\langle\psi \circ f^{n} L^{n}\left(T_{m}^{+}\right), \omega^{k-s}\right\rangle=\left\langle T_{m}^{+} \wedge \Lambda^{n}\left(\omega^{k-s}\right), \psi\right\rangle
$$

and let $m$ go to $\infty$. So we have $\left\langle\psi \circ f^{n} T_{s}^{+}, \omega^{k-s}\right\rangle=\left\langle T_{s}^{+} \wedge \Lambda^{n}\left(\omega^{k-s}\right), \psi\right\rangle$ because $T_{s}^{+} \wedge \omega^{k-s}$ gives no mass to $I\left(f^{n}\right)$.

By Theorem 3.4.1, we have that $T_{s}^{+} \wedge \Lambda^{n}\left(\omega^{k-s}\right)$ converges (in the Hartogs' sense hence in the sense of currents) to $\mu$ which means that $c=\mu(\psi)$. In particular, $c$ does not depend on the choice of $S$ and the first part of the lemma follows.

Now we show that $\left\|d d^{c}\left(\psi \circ f^{n} T_{s}^{+}\right)\right\|$goes to zero. Let $\Theta$ be a test form of bidegree $(k-s-1, k-s-1)$. Again, we consider a smooth approximation of $T_{s}^{+}$that we denote $T_{m}^{+}$. Using the fact that $\left(\psi \circ f^{n}\right) L^{n}\left(T_{m}^{+}\right)=d^{-s n}\left(f^{n}\right)^{*}\left(\psi T_{m}^{+}\right)$, we compute:

$$
\begin{aligned}
\left\langle\psi \circ f^{n} L^{n}\left(T_{m}^{+}\right), d d^{c} \Theta\right\rangle & \left.=\left\langle d^{-s n}\left(f^{n}\right)^{*}\left(\psi T_{m}^{+}\right), d d^{c} \Theta\right)\right\rangle \\
& =\left\langle d^{-s n}\left(f^{n}\right)^{*}\left(d d^{c}(\psi) \wedge T_{m}^{+}\right), \Theta\right\rangle .
\end{aligned}
$$

Writing $\Theta=\Theta^{+}-\Theta^{-}$we can assume that $\Theta$ is positive (so $\Theta \leq B \omega^{k-s-1}$ with $B>0$ large enough which depends only on $\|\Theta\|)$. Let $A>0$ be such that $-A \omega \leq d d^{c} \psi \leq A \omega$. It is sufficient to control:

$$
\left\langle d^{-s n}\left(f^{n}\right)^{*}\left(\omega \wedge T_{m}^{+}\right), \omega^{k-s-1}\right\rangle .
$$

We recognize that this is equal by definition to $d_{s+1}^{n} d^{-s n}=\delta^{-n}$. We let $m$ go to $\infty$ and we have that $\left\langle d d^{c}\left(\psi \circ f^{n} \wedge T_{s}^{+}\right), \Theta\right\rangle$ goes to 0 with $n$ uniformly on $\|\Theta\|$.

ThEOREM 3.4.15. - The measure $\mu$ is mixing.

Proof. - Let $\psi$ and $\varphi$ be real smooth functions on $\mathbb{P}^{k}$. We can assume without loss of generality that $0 \leq \psi, \varphi \leq 1$. Then for $S$ in $\mathscr{C}_{k-s}$ smooth, we have by the above lemma that:

$$
\left\langle\left(\varphi \psi \circ f^{n}\right) T_{s}^{+}, S\right\rangle
$$

converges to:

$$
\mu(\psi)\left\langle\varphi T_{s}^{+}, S\right\rangle .
$$

We consider a sequence $\left(T_{m}^{-}\right)$of smooth currents in $\mathscr{C}_{1}$ converging in the Hartogs' sense to $T^{-}$(the Green current of order 1 of $\left.f^{-1}\right)$. Then let $m=\left(m_{1}, m_{2}, \ldots, m_{k-s}\right)$ 
and $m^{\prime}=\left(m_{1}^{\prime}, m_{2}^{\prime}, \ldots, m_{k-s}^{\prime}\right)$ in $\mathbb{N}^{k-s}$. We have that $T_{m_{1}}^{-} \wedge \cdots \wedge T_{m_{k-s}}^{-}$converges to $T_{k-s}^{-}$in the Hartogs' sense when the $m_{i}$ go to $\infty$. We decompose:

$$
T_{m_{1}}^{-} \wedge \cdots \wedge T_{m_{k-s}}^{-}-T_{m_{1}^{\prime}}^{-} \wedge \cdots \wedge T_{m_{k-s}^{\prime}}^{-}
$$

as:

$$
\begin{array}{r}
\left(T_{m_{1}}^{-}-T_{m_{1}^{\prime}}^{-}\right) \wedge T_{m_{2}}^{-} \wedge \cdots \wedge T_{m_{k-s}}^{-}+ \\
T_{m_{1}^{\prime}}^{-} \wedge\left(T_{m_{2}}^{-}-T_{m_{2}^{\prime}}^{-}\right) \wedge \cdots \wedge T_{m_{k-s}}^{-}+ \\
\ldots \\
T_{m_{1}^{\prime}}^{-} \wedge \cdots \wedge T_{m_{k-s-1}^{\prime}}^{-} \wedge\left(T_{m_{k-s}}^{-}-T_{m_{k-s}^{\prime}}^{-}\right) .
\end{array}
$$

As in the previous lemma, let $\left(v_{j}\right)$ be an increasing sequence of smooth qpsh $\left(i \partial \bar{\partial} v_{j}+\right.$ $\omega \geq 0$ ) functions with $0 \leq v_{j} \leq 1$ converging uniformly to 1 on the compact sets of $\mathbb{P}^{k} \backslash \mathscr{C}\left(f^{n}\right)$ and equal to 0 on some neighborhood of $\mathscr{C}\left(f^{n}\right)$.

We also define $v_{j}^{\prime}:=\alpha \circ v_{j}$ with $\alpha:[0,1] \rightarrow[0,1]$ a smooth function equal to 0 in $[0,1 / 3]$ and to 1 in $[2 / 3,1]$ so that the sequence of functions $v_{j}^{\prime}$ is equal to 1 on the compact sets of $\mathbb{P}^{k} \backslash \mathscr{C}\left(f^{n}\right)$ for $j$ large enough and is equal to 0 on some neighborhood of $\mathscr{C}\left(f^{n}\right)$.

We consider the quantity $\left\langle v_{j}^{\prime} \varphi \psi \circ f^{n} T_{s}^{+},\left(T_{m_{1}}^{-}-T_{m_{1}^{\prime}}^{-}\right) \wedge \cdots \wedge T_{m_{k-s}}^{-}\right\rangle$. Write $T_{i}^{-}=$ $\omega+d d^{c} g_{i}$ where the $g_{i}$ are decreasing. By Stokes' formula, we have that:

$$
\begin{array}{r}
\left\langle v_{j}^{\prime} \varphi \psi \circ f^{n} T_{s}^{+},\left(T_{m_{1}}^{-}-T_{m_{1}^{\prime}}^{-}\right) \wedge \cdots \wedge T_{m_{k-s}}^{-}\right\rangle= \\
-\left\langle\left(\varphi \psi \circ f^{n} d v_{j}^{\prime}+v_{j}^{\prime} \psi \circ f^{n} d \varphi+v_{j}^{\prime} \varphi d \psi \circ f^{n}\right) \wedge T_{s}^{+}, d^{c}\left(g_{m_{1}}-g_{m_{1}^{\prime}}\right) \wedge \cdots \wedge T_{m_{k-s}}^{-}\right\rangle .
\end{array}
$$

Write the last sum $I+I I+I I I$ with obvious notations. Using Cauchy Schwarz inequality for the first term, we have that:

$$
\begin{array}{r}
|I|^{2} \leq\left\langle d v_{j} \wedge d^{c} v_{j} \wedge T_{s}^{+}, T_{m_{2}}^{-} \wedge \cdots \wedge T_{m_{k-s}}^{-}\right\rangle \times \\
\left\langle\left(\alpha^{\prime}\left(v_{j}\right)\right)^{2} d\left(g_{m_{1}}-g_{m_{1}^{\prime}}\right) \wedge d^{c}\left(g_{m_{1}}-g_{m_{1}^{\prime}}\right) \wedge T_{s}^{+}, T_{m_{2}}^{-} \wedge \cdots \wedge T_{m_{k-s}}^{-}\right\rangle .
\end{array}
$$

As in the proof of the previous lemma, we have that this term goes to zero when $j \rightarrow \infty$ since $\alpha^{\prime}\left(v_{j}\right)$ converges uniformly to 0 on the compact sets of $\mathbb{P}^{k} \backslash \mathscr{C}\left(f^{n}\right)$.

Now for $I I$, we use Cauchy Schwarz inequality and we have that:

$$
\begin{aligned}
|I I|^{2} \leq & \left\langle d \varphi \wedge d^{c} \varphi \wedge T_{s}^{+}, T_{m_{2}}^{-} \wedge \cdots \wedge T_{m_{k-s}}^{-}\right\rangle \\
& \left\langle d\left(g_{m_{1}}-g_{m_{1}^{\prime}}\right) \wedge d^{c}\left(g_{m_{1}}-g_{m_{1}^{\prime}}\right) \wedge T_{s}^{+}, T_{m_{2}}^{-} \wedge \cdots \wedge T_{m_{k-s}}^{-}\right\rangle .
\end{aligned}
$$


The first term of the product is bounded as it converges to $\int d \varphi \wedge d^{c} \varphi \wedge T_{s}^{+} \wedge\left(T^{-}\right)^{s-1}$. By Stokes, we recognize that the second term is equal to

$$
\begin{aligned}
& \left\langle-\left(g_{m_{1}}-g_{m_{1}^{\prime}}\right) \wedge d d^{c}\left(g_{m_{1}}-g_{m_{1}^{\prime}}\right) \wedge T_{s}^{+}, T_{m_{2}}^{-} \wedge \cdots \wedge T_{m_{k-s}}^{-}\right\rangle= \\
& \left\langle-\left(g_{m_{1}}-g_{m_{1}^{\prime}}\right) \wedge\left(T_{m_{1}}^{-}-T_{m_{1}^{\prime}}^{-}\right) \wedge T_{s}^{+}, T_{m_{2}}^{-} \wedge \cdots \wedge T_{m_{k-s}}^{-}\right\rangle= \\
& \mathcal{U}_{T_{m_{1}}^{-}}\left(T_{s}^{+} \wedge T_{m_{1}^{\prime}}^{-} \wedge T_{m_{2}}^{-} \wedge \cdots \wedge T_{m_{k-s}}^{-}\right)-\mathcal{U}_{T_{m_{1}^{\prime}}^{-}}\left(T_{s}^{+} \wedge T_{m_{1}^{\prime}}^{-} \wedge T_{m_{2}}^{-} \wedge \cdots \wedge T_{m_{k-s}}^{-}\right)+ \\
& \mathcal{U}_{T_{m_{1}^{\prime}}^{-}}\left(T_{s}^{+} \wedge T_{m_{1}}^{-} \wedge T_{m_{2}}^{-} \wedge \cdots \wedge T_{m_{k-s}}^{-}\right)-\mathcal{U}_{T_{m_{1}}^{-}}\left(T_{s}^{+} \wedge T_{m_{1}}^{-} \wedge T_{m_{2}}^{-} \wedge \cdots \wedge T_{m_{k-s}}^{-}\right) \text {. }
\end{aligned}
$$

Observe that this term goes to 0 when the $m_{i}, m_{i}^{\prime}$ are large enough. Indeed recall that $\mathcal{U}_{1}(S, T)$ is continuous for the Hartogs' convergence (Lemma A.1.11), so:

$$
\mathcal{U}_{T_{m_{1}}^{-}}\left(T_{s}^{+} \wedge T_{m_{1}^{\prime}}^{-} \wedge T_{m_{2}}^{-} \wedge \cdots \wedge T_{m_{k-s}}^{-}\right)
$$

converges to $U_{T^{-}}(\mu)$ which is finite and so does the other terms in the majoration of $I I$ (the convergence is uniform else we could extract a subsequence which does not converge).

Now we bound III. Applying Cauchy-Schwarz inequality gives:

$$
\begin{aligned}
|I I I|^{2} \leq & \left\langle\left|v_{j}^{\prime}\right|^{2} d \psi \circ f^{n} \wedge d^{c} \psi \circ f^{n} \wedge T_{s}^{+}, T_{m_{2}}^{-} \wedge \cdots \wedge T_{m_{k-s}}^{-}\right\rangle \\
& \left\langle d\left(g_{m_{1}}-g_{m_{1}^{\prime}}\right) \wedge d^{c}\left(g_{m_{1}}-g_{m_{1}^{\prime}}\right) \wedge T_{s}^{+}, T_{m_{2}}^{-} \wedge \cdots \wedge T_{m_{k-s}}^{-}\right\rangle
\end{aligned}
$$

Observe that the second integral is the same than in the bound of $I I$ so it goes to zero. For the first term of the product, we use that $f^{n}$ is smooth in the support of $v_{j}^{\prime}$ and thus $d \psi \circ f^{n} \wedge d^{c} \psi \circ f^{n}=\left(f^{n}\right)^{*}\left(d \psi \wedge d^{c} \psi\right)$ in the integral. We can assume that $d \psi \wedge d^{c} \psi \leq \omega$. Using the invariance of $T_{s}^{+}$and the fact that $v_{j}^{\prime}$ is equal to 0 near $\mathscr{C}\left(f^{n}\right)$, we have that $\left(f^{n}\right)^{*}(\omega) \wedge T_{s}^{+}=d^{-s n}\left(f^{n}\right)^{*}\left(\omega \wedge T_{s}^{+}\right)$in the integral, so the first term in the bound of $I I I$ is less than:

$$
\frac{1}{d^{s n}}\left\langle\left(f^{n}\right)^{*}\left(\omega \wedge T_{s}^{+}\right), T_{m_{2}}^{-} \wedge \cdots \wedge T_{m_{k-s}}^{-}\right\rangle .
$$

That last term can be computed cohomologically and is equal to $\frac{\delta^{n(k-s-1)}}{d^{n s}}<1$. So as for $I I$, we have that $I I I$ goes to 0 uniformly in $n$.

Letting $j$ go to $\infty$, we have that

$$
\left\langle\varphi \psi \circ f^{n} T_{s}^{+}, T_{m_{1}}^{-} \wedge \cdots \wedge T_{m_{k-s}}^{-}-T_{m_{1}^{\prime}}^{-} \wedge \cdots \wedge T_{m_{k-s}^{\prime}}^{-}\right\rangle
$$

converges uniformly to 0 . In particular, we can interchange the limit in:

$$
\lim _{m} \lim _{n}\left\langle\left(\varphi \psi \circ f^{n}\right) T_{s}^{+}, T_{m_{1}}^{-} \wedge \cdots \wedge T_{m_{k-s}}^{-}\right\rangle
$$

which gives $\lim _{n} \mu\left(\varphi \psi \circ f^{n}\right)=\mu(\varphi) \mu(\psi)$ hence the mixing. 
We now show that the measure $\mu$ satisfies the hypothesis of Chapter 2 and we deduce from that a bound of its entropy. Recall that we denote by $\mu_{n}$ the sequence of probabilities:

$$
\mu_{n}:=\frac{1}{n} \sum_{i=0}^{n-1} f_{*}^{i}\left(\frac{\left(f^{n}\right)^{*} \omega^{s} \wedge \omega^{k-s}}{\lambda_{s}\left(f^{n}\right)}\right) .
$$

In our case, using Lemma A.2.2 and hypothesis $(H)$, we can write it as:

$$
\mu_{n}=\frac{1}{n} \sum_{i=0}^{n-1} L^{n-i}\left(\omega^{s}\right) \wedge \Lambda^{i}\left(\omega^{k-s}\right) .
$$

We consider the hypothesis $(H)$ : there exists a subsequence $\mu_{\psi(n)}$ of $\mu_{n}$ converging to a measure $\mu^{\prime}$ such that:

$$
(H): \lim _{n \rightarrow+\infty} \int \log d(x, I) d \mu_{\psi(n)}(x)=\int \log d(x, I) d \mu^{\prime}(x)>-\infty .
$$

In here, we do not need to take a subsequence:

Proposition 3.4.16. - The sequence $\left(\mu_{n}\right)$ converges to $\mu$ and satisfies the hypothesis $(H)$.

Proof. - Let $\varphi$ be a smooth test function. Choose $\varepsilon>0$. By Theorem 3.4.1, since $L^{n-i}\left(\omega^{s}\right)$ and $\Lambda^{i}\left(\omega^{k-s}\right)$ converge in the Hartogs' sense, Proposition A.1.15 assures us that $L^{n-i}\left(\omega^{s}\right) \wedge \Lambda^{i}\left(\omega^{k-s}\right)$ converges in the Hartogs' sense to $\mu$. So we have for $\sqrt{n} \leq i \leq n-\sqrt{n}$ and $n$ large enough that $\left|L^{n-i}\left(\omega^{s}\right) \wedge \Lambda^{i}\left(\omega^{k-s}\right)(\varphi)-\mu(\varphi)\right| \leq \varepsilon$. The fact that $\left(\mu_{n}\right)$ goes to $\mu$ follows since they are $o(n)$ terms for which the estimation does not stand.

Now, by Lemma A.2.5, we see that there exist constants $A_{i, n} \geq 0$ such that $\mathcal{U}_{L^{n-i}\left(\omega^{s}\right) \wedge \Lambda^{i}\left(\omega^{k-s}\right)} \geq \mathcal{U}_{\mu}-A_{i, n}$ with $A_{i, n}$ uniformly bounded from above by $C$ and arbitrarily close to zero for $i$ and $n$ large enough. We consider super-potentials of mean 0. In particular:

$$
u_{\mu_{n}} \geq u_{\mu}-\frac{1}{n} \sum_{i=0}^{n-1} A_{i, n} .
$$

So we have that the sequence $\mu_{n}$ is more H-regular than $\mu$ for all $n$. We also have the convergence in the Hartogs' sense to $\mu$ since $\frac{1}{n} \sum_{i=0}^{n-1} A_{i, n}$ goes to 0 when $n \rightarrow+\infty$.

Thus $\mu_{n}(G) \rightarrow \mu(G)$ which is finite by Theorem 3.4.1 where $G$ is a negative potential of the Green current of order 1 that we denote $T^{+}$. Since $T^{+}$is less H-regular than $L(\omega)$, we have that if $U_{L(\omega)}$ is a quasi-potential of $L(\omega)$ then $\mu_{n}\left(U_{L(\omega)}\right) \rightarrow \mu\left(U_{L(\omega)}\right)$ which is also finite. By Lemma 3.2.4, we have that:

$$
A U_{L(\omega)}(x)<\log \operatorname{dist}\left(x, I^{+}\right)
$$


for $A>0$ large enough. We denote $\varphi:=\log \operatorname{dist}\left(x, I^{+}\right)$. Since $\mu$ gives no mass to $I^{+}$ that means that $A U_{L(\omega)}(x) \leq \varphi \leq 0$ for $\mu$ a.e point, so we have that $\varphi \in L^{1}(\mu)$. We have the classical lemma:

LEMMA 3.4.17. - Let $\nu_{n}$ be a sequence of measures converging to $\nu$ in the sense of measures. Then for $v$ an upper semi-continuous function, we have that

$$
\lim \sup \nu_{n}(v) \leq \nu(v) .
$$

Proof. - Recall that an usc function can be written as the limit of a decreasing sequence of continuous functions. So for some small $\alpha>0$ we can take $v^{\prime} \geq v$ a continuous function such that $\int v^{\prime} d \nu \leq \int v d \nu+\alpha$ by monotone convergence. In particular:

$$
\int v d \nu_{n} \leq \int v^{\prime} d \nu_{n} \rightarrow \int v^{\prime} d \nu \leq \int v d \nu+\alpha
$$

And the result follows by letting $\alpha \rightarrow 0$.

End of the proof of the proposition. - Now, $\varphi$ is upper semi-continuous, so:

$$
\lim \sup \mu_{n}(\varphi) \leq \mu(\varphi)
$$

We also have that $(A+1) U_{L(\omega)}-\varphi$ is upper semi-continuous (we use the fact that it is equal to $-\infty$ on $\left.I^{+}\right)$. That and $\mu_{n}\left(U_{L(\omega)}\right) \rightarrow \mu\left(U_{L(\omega)}\right)$ give:

$$
\liminf \mu_{n}(\varphi) \geq \mu(\varphi) \text {. }
$$

This is exactly the fact that $\mu$ satisfies Hypothesis $(\mathrm{H})$.

We can now apply Theorem 1 to get the proposition:

TheOREM 3.4.18. - The topological entropy of $f$ is greater than $\log d_{s}=s \log d$. More precisely, the entropy of $\mu$ is greater than $s \log d$.

On the other hand, the topological entropy is always bounded by $\max _{0 \leq s \leq k} \log d_{s}$ (see [25] for the projective case and [22] for the Kähler case). So we have the fundamental result:

TheOREM 3.4.19. - The topological entropy of $f$ is equal to $\log d_{s}$. Moreover, the entropy of $\mu$ is equal to $s \log d$ so $\mu$ is a measure of maximal entropy.

REMARK 3.4.20. - A very natural question asked by the referee is whether $\mu$ is unique. To answer such a question one often use some sort of control on the size of the stable and unstable manifolds that seems very difficult to get here (see for example [2] in the case of Hénon maps). 
This allows us to use the first author's estimate of the Lyapunov exponents (Corollary 3 in [11]). To apply that result, we need to have that $\log \left(\operatorname{dist}\left(x, \mathscr{C}^{+}\right)\right)$is integrable with respect to $\mu$. For that observe that the function $U_{L(\omega)}$ is integrable with respect to $\mu$. By invariance, $f_{*}\left(U_{L(\omega)}\right)$ is also integrable. Write $U_{L(\omega)}$ as in Lemma 3.2.4:

$$
U_{L(\omega)}=d^{-1} \log |F|^{2}-\log |Z|^{2},
$$

where $f=\left[P_{1}: \ldots: P_{k+1}\right]$ and $F=\left(P_{1}, \ldots, P_{k+1}\right)$. Write $f^{-1}=\left[Q_{1}: \ldots\right.$ : $\left.Q_{k+1}\right]$ where the $Q_{i}$ are homogeneous polynomials of degree $\delta$ and write $F^{-1}=$ $\left(Q_{1}, \ldots, Q_{k+1}\right)$. There is of course an abuse of notation since $F \circ F^{-1} \neq I d$ instead, we have that:

$$
F \circ F^{-1}=P\left(z_{1}, \ldots, z_{k+1}\right) \times\left(z_{1}, \ldots, z_{k+1}\right),
$$

where $P$ is an homogeneous polynomial of degree $d \delta-1$ equal to 0 in $\pi^{-1}\left(\mathscr{C}^{-}\right)$and $\pi: \mathbb{C}^{k+1} \rightarrow \mathbb{P}^{k}$ is the canonical projection. Then, we have that:

$$
f_{*}\left(\frac{1}{d} \log |F|^{2}-\log |Z|^{2}\right)=\frac{1}{d} \log \left|F \circ F^{-1}\right|^{2}-\log \left|F^{-1}(Z)\right|^{2} .
$$

We recognize $d^{-1} \log \left|F \circ F^{-1}\right|^{2}-\delta \log |Z|^{2}+\delta \log |Z|^{2}-\log \left|F^{-1}(Z)\right|^{2}$. But

$$
\delta \log |Z|^{2}-\log \left|F^{-1}(Z)\right|^{2}=-\delta U_{\Lambda(\omega)}
$$

is in $L^{1}(\mu)$ and by difference, so is $d^{-1} \log \left|F \circ F^{-1}\right|^{2}-\delta \log |Z|^{2}$. As in Lemma 3.2.4, we then have that $\log \operatorname{dist}\left(., \mathscr{C}^{-}\right)$is in $L^{1}(\mu)$. Similarly, $\log \operatorname{dist}\left(., \mathscr{C}^{+}\right)$is in $L^{1}(\mu)$.

ThEOREM 3.4.21. - The Lyapunov exponents $\chi_{1} \geq \chi_{2} \geq \cdots \geq \chi_{k}$ of $\mu$ are well defined and we have the estimates:

$$
\begin{array}{r}
\chi_{1} \geq \cdots \geq \chi_{s} \geq \frac{1}{2} \log \frac{d_{s}}{d_{s-1}}=\frac{1}{2} \log d>0 \\
0>-\frac{1}{2} \log \delta=\frac{1}{2} \log \frac{d_{s+1}}{d_{s}} \geq \chi_{s+1} \geq \cdots \geq \chi_{k} .
\end{array}
$$

In particular, the measure $\mu$ is hyperbolic. 


\section{APPENDIX A}

\section{SUPER-POTENTIALS}

\section{A.1. Definitions and properties of super-potentials}

We recall here the facts and definitions we use on super-potentials. Everything in this section was taken from [31] so we refer the reader to this paper for proofs and details.

Recall that $\mathscr{C}_{s}$ is the convex compact set of (strongly) positive closed currents $S$ of bidegree $(s, s)$ on $\mathbb{P}^{k}$ and of mass 1 . To develop the calculus, we have to consider $\mathscr{C}_{s}$ as an infinite dimensional space with special families of currents that we parametrize by the unit disc $\Delta$ in $\mathbb{C}$. We call these families special structural discs of currents. The notion of structural varieties of $\mathscr{C}_{s}$ was introduced in [28]. In some sense, we consider $\mathscr{C}_{s}$ as a space of infinite dimension admitting "complex subvarieties" of finite dimension. For $S$ in $\mathscr{C}_{s}$, it is always possible to construct a special structural variety $\varphi: \Delta \rightarrow \mathscr{C}_{s}$ such that $\varphi(0)=S$ and $\varphi(z)$ is a smooth form for $z \neq 0$. The points of the structural disk centered at $R$ are defined as the regularization of $R$ by an approximation of the identity in $\operatorname{Aut}\left(\mathbb{P}^{k}\right)$; the structural disk can be seen as a positive closed current in $\Delta \times \mathbb{P}^{k}$ and the points of the disks are the vertical slices of that current.

Let $S$ be a current in $\mathscr{C}_{s}$ with $s \geq 1$. If $U$ is a $(s-1, s-1)$-current such that $d d^{c} U=S-\omega^{s}$, we say that $U$ is a quasi-potential of $S$. The integral $\left\langle U, \omega^{k-s+1}\right\rangle$ is the mean of $U$. Observe that such quasi-potential is defined up to a $d d^{c}$-closed current. For $s=1$ such functions are constant a.e., but in the general case, they can be singular currents. Nevertheless, we have the proposition:

Proposition A.1.1. - Let $S$ be a current in $\mathscr{C}_{s}$. Then, there is a negative quasipotential $U$ of $S$ depending linearly on $S$ such that for every $r$ with $1 \leq r<k /(k-1)$ and for $1 \leq \rho<2 k /(2 k-1)$

$$
\|U\|_{\mathscr{L}^{r}} \leq c_{r} \quad \text { and } \quad\|d U\|_{\mathscr{L}^{\rho}} \leq c_{\rho}
$$


for some positive constants $c_{r}, c_{\rho}$ independent of $S$. Moreover, $U$ depends continuously on $S$ with respect to the $\mathcal{L}^{r}$ topology on $U$ and the weak topology on $S$.

We are going to introduce a super-potential associated to $S$. It is an affine upper semi-continuous function $\mathcal{U}_{S}$ defined on $\mathscr{C}_{k-s+1}$ with values in $\mathbb{R} \cup\{-\infty\}$. For $R \in$ $\mathscr{C}_{k-s+1}$ smooth, we define the super-potential of mean $M$ of $S$ by $\mathcal{U}_{S}(R):=\left\langle S, U_{R}\right\rangle$ where $U_{R}$ is a quasi-potential of $R$ of mean $\left\langle U_{R}, \omega^{s}\right\rangle=M$. The integral $\left\langle S, U_{R}\right\rangle$ does not depend on the choice of $U_{R}$ with a fixed mean $M$. If $S$ is smooth, we have $\mathcal{U}_{S}(R)=\left\langle U_{S}, R\right\rangle$ where $U_{S}$ is a quasi-potential of $S$ of mean $M$. Now assume that $R$ is not smooth. Consider the above special structural variety $\varphi: \Delta \rightarrow \mathscr{C}_{k-s+1}$ associated to $R \in \mathscr{C}_{k-s+1}$ and write $R_{\theta}$ for $\varphi(\theta)$. Then the function $u(\theta):=\mathcal{U}_{S}\left(R_{\theta}\right)$ defined on $\Delta^{*}$ can be extended as a quasi-subharmonic function on $\Delta$. Let $\left(S_{\theta}\right)$ and let $\left(R_{\theta}\right)$ be special structural disks associated to $S \in \mathscr{C}_{s}$ and $R \in C_{k-s+1}$. Then we have the proposition:

Proposition A.1.2. - The function $\mathcal{U}_{S}$ can be extended in a unique way to an affine upper semi continuous function on $\mathscr{C}_{k-s+1}$ with values in $\mathbb{R} \cup\{-\infty\}$, also denoted by $\mathcal{U}_{S}$, such that

In particular, we have

$$
\mathcal{U}_{S}(R)=\lim _{\theta \rightarrow 0} \mathcal{U}_{S_{\theta}}(R)=\lim _{\theta \rightarrow 0} \mathcal{U}_{S}\left(R_{\theta}\right) .
$$

$$
U_{S}(R)=\limsup _{R^{\prime} \rightarrow R} \mathcal{U}_{S}\left(R^{\prime}\right) \text { with } R^{\prime} \text { smooth. }
$$

Moreover, there is a constant $c \geq 0$ independent of $S$ such that if $\mathcal{U}_{S}$ is the superpotential of mean $m$ of $S$, then $\mathcal{U}_{S} \leq m+c$ everywhere.

Super-potentials determine the current, more precisely, we have the proposition:

Proposition A.1.3. - Let I be a compact subset in $\mathbb{P}^{k}$ with $(2 k-2 s)$-dimensional Hausdorff measure 0 . Let $S, S^{\prime}$ be currents in $\mathscr{C}_{s}$ and $\mathcal{U}_{S}, \mathcal{U}_{S^{\prime}}$ be super-potentials of $S, S^{\prime}$. If $\mathcal{U}_{S}=\mathcal{U}_{S^{\prime}}$ on smooth forms in $\mathscr{C}_{k-s+1}$ with compact support in $\mathbb{P}^{k} \backslash I$, then $S=S^{\prime}$.

For $I=\varnothing$, this tells us that the values of the super-potential on smooth forms determine uniquely the current.

A crucial notion to prove the convergence of currents is the following:

Definition A.1.4. - Let $\left(S_{n}\right)$ be a sequence in $\mathscr{C}_{s}$ converging to a current $S$. Let $\mathcal{U}_{S_{n}}\left(\right.$ resp. $\left.\mathcal{U}_{S}\right)$ be the super-potential of mean $M_{n}$ (resp. M) of $S_{n}$ (resp. S). Assume that $M_{n}$ converge to $M$. If $\mathcal{U}_{S_{n}} \geq \mathcal{U}_{S}$ for every $n$, we say that $\left(S_{n}\right)$ converge to $S$ in the Hartogs' sense. If a current $S^{\prime}$ in $\mathscr{C}_{s}$ admits a super-potential $\mathcal{U}_{S^{\prime}}$ such that $\mathcal{U}_{S^{\prime}} \geq \mathcal{U}_{S}$ we say that $S^{\prime}$ is more H-regular than $S$. 
Smooth currents are dense for the Hartog's convergence, more precisely:

Proposition A.1.5. - Let $S \in \mathscr{C}_{s}$ and let $\mathcal{U}$ be a super-potential of $S$ of mean $M$. There is a sequence of smooth forms $\left(S_{n}\right)$ in $\mathscr{C}_{s}$ with super-potentials $\mathcal{U}_{n}$ of mean $M_{n}$ such that

- $\operatorname{supp}\left(S_{n}\right)$ converge to $\operatorname{supp}(S)$;

- $S_{n}$ converge to $S$ and $M_{n} \rightarrow M$;

- $\left(\mathcal{U}_{n}\right)$ decreases to $\mathcal{U}$.

We have the following convergence theorem:

Proposition A.1.6. - Let $\left(S_{n}\right)$ be a sequence in $\mathscr{C}_{s}$ converging to a current $S$. Let $\mathcal{U}_{S_{n}}\left(\right.$ resp. $\left.\mathcal{U}_{S}\right)$ be the super-potential of mean $M_{n}$ (resp. $M$ ) of $S_{n}$ (resp. $S$ ). Assume that $M_{n}$ converge to $M$. Let $\mathcal{U}$ be a continuous function on a compact subset $K$ of $\mathscr{C}_{k-s+1}$ such that $\mathcal{U}_{S}<\mathcal{U}$ on $K$. Then, for $n$ large enough we have $\mathcal{U}_{S_{n}}<\mathcal{U}$ on $K$. In particular, we have limsup $\mathcal{U}_{S_{n}} \leq \mathcal{U}_{S}$ on $\mathscr{C}_{k-s+1}$. Furthermore, if $S_{n} \rightarrow S$ in the Hartogs' sense, then $\mathcal{U}_{S_{n}} \rightarrow \mathcal{U}_{S}$ pointwise.

In $\mathscr{C}_{k-s+1}$, they are points which are more "regular" than other, namely smooth forms. This is a difference with psh functions. In particular, it is often easier to obtain the convergence at such points:

Proposition A.1.7. - Let $\left(S_{n}\right)$ be a sequence in $\mathscr{C}_{s}$ and $\mathcal{U}_{S_{n}}$ be super-potentials of mean $M_{n}$ of $S_{n}$. Assume that $\left(\mathcal{U}_{S_{n}}\right)$ converges to a finite function $\mathcal{U}$ on smooth forms in $\mathscr{C}_{k-s+1}$. Then, $\left(M_{n}\right)$ converges to a constant $M,\left(S_{n}\right)$ converges to a current $S$ and $\mathcal{U}$ is equal to the super-potential of mean $M$ of $S$ on smooth forms in $\mathscr{C}_{k-s+1}$.

The following is the main argument to get the convergence of the Green current:

Proposition A.1.8. - Let $\mathcal{U}_{S_{n}}$ be super-potentials of mean $M_{n}$ of $S_{n}$. Assume that $\mathcal{U}_{S_{n}}$ decrease to a function $\mathcal{U} \neq-\infty$. Then, $\left(S_{n}\right)$ converges to a current $S,\left(M_{n}\right)$ converges to a constant $M$ and $\mathcal{U}$ is the super-potential of mean $M$ of $S$.

In particular, the convergence at one point of the super-potentials gives the convergence of the currents in the Hartogs' sense in the case of decreasing super-potentials.

An interesting symmetry result is that if $\mathcal{U}_{S}$ and $\mathcal{U}_{R}$ are super-potentials of the same mean $M$ of $R$ and $S$ respectively, then $\mathcal{U}_{S}(R)=\mathcal{U}_{R}(S)$.

There is a notion of super-polarity for Borel subsets $E$ of $\mathscr{C}_{k-s+1}$. This notion does not describe "small" sets $E$ but rather how singular are the currents in $E$.

Definition A.1.9. - We say that $E$ is super-polar in $\mathscr{C}_{k-s+1}$ if there is a superpotential $\mathcal{U}_{S}$ of a current $S$ in $\mathscr{C}_{s}$ such that $E \subset\left\{\mathcal{U}_{S}=-\infty\right\}$. 
Denote by $\widetilde{E}$ the set of currents $c R+(1-c) R^{\prime}$ with $R \in \widehat{E}, R^{\prime} \in \mathscr{C}_{k-s+1}$ and $0<c \leq 1$, and $\widehat{E}$ the barycentric hull of $E$, i.e. the set of currents $\int R d \nu(R)$ where $\nu$ is a probability measure on $\mathscr{C}_{k-s+1}$ such that $\nu(E)=1$. Then, $\widetilde{E}$ and $\widehat{E}$ are convex.

Proposition A.1.10. - The following properties are equivalent

1. E is super-polar in $\mathscr{C}_{k-s+1}$.

2. $\widehat{E}$ is super-polar in $\mathscr{C}_{k-s+1}$.

3. $\widetilde{E}$ is super-polar in $\mathscr{C}_{k-s+1}$.

Moreover, a countable union of super-polar sets is super-polar.

One of the purposes of super-potentials is to define the wedge product of current (see Section 4 in [31]). We define a universal function $\mathcal{U}_{s}$ on $\mathscr{C}_{s} \times \mathscr{C}_{k-s+1}$ by

$$
\mathcal{U}_{s}(S, R):=\mathcal{U}_{S}(R)=\mathcal{U}_{R}(S)
$$

where $\mathcal{U}_{S}$ and $\mathcal{U}_{R}$ are super-potentials of mean 0 of $S$ and $R$. The function $\mathcal{U}_{s}$ is is u.s.c. on $\mathscr{C}_{s} \times \mathscr{C}_{k-s+1}$. It even enjoys a nice continuity for the Hartogs' convergence:

LEMMA A.1.11. - Let $\left(S_{n}\right)_{n \geq 0}$ and $\left(R_{n}\right)_{n \geq 0}$ be sequences of currents in $\mathscr{C}_{s}$ and $C_{k-s+1}$ converging in the Hartogs' sense to $S$ and $R$ respectively. Then, $\mathcal{U}_{s}\left(S_{n}, R_{n}\right)$ converge to $\mathcal{U}_{s}(S, R)$. Moreover, if $\mathcal{U}_{s}(S, R)$ is finite, then $\mathcal{U}_{s}\left(S_{n}, R_{n}\right)$ is finite for every $n$.

We have the proposition:

Proposition A.1.12. - Let $s_{1} \in \mathbb{N}^{*}$ and $s_{2} \in \mathbb{N}^{*}$ with $s_{1}+s_{2} \leq k$. The following conditions are equivalent and are symmetric on $R_{1} \in \mathscr{C}_{s_{1}}$ and $R_{2} \in \mathscr{C}_{s_{2}}$ :

1. $\mathcal{U}_{s_{1}}\left(R_{1}, R_{2} \wedge \Omega\right)$ is finite for at least one smooth form $\Omega$ in $\mathscr{C}_{k-s_{1}-s_{2}+1}$.

2. $\mathcal{U}_{s_{1}}\left(R_{1}, R_{2} \wedge \Omega\right)$ is finite for every smooth form $\Omega$ in $\mathscr{C}_{k-s_{1}-s_{2}+1}$.

3. There are sequences $\left(R_{i, n}\right)_{n \geq 0}$ in $\mathscr{C}_{s_{i}}$ converging to $R_{i}$ and a smooth form $\Omega$ in $\mathscr{C}_{k-s_{1}-s_{2}+1}$ such that $\mathcal{U}_{s_{1}}\left(R_{1, n}, R_{2, n} \wedge \Omega\right)$ is bounded.

Definition A.1.13. - We say that $R_{1}$ and $R_{2}$ are wedgeable if they satisfy the conditions in Proposition A.1.12.

Assume that $R_{1} \in \mathscr{C}_{s_{1}}$ and $R_{2} \in \mathscr{C}_{s_{2}}$ are wedgeable. For every smooth real form $\varphi$ of bidegree $\left(k-s_{1}-s_{2}, k-s_{1}-s_{2}\right)$, write $d d^{c} \varphi=c\left(\Omega^{+}-\Omega^{-}\right)$where $\Omega^{ \pm}$are smooth forms in $\mathscr{C}_{k-s_{1}-s_{2}+1}$ and $c$ is a positive constant. We define the wedge-product (or the intersection) $R_{1} \wedge R_{2}$ by its action on the smooth forms by:

$$
\left\langle R_{1} \wedge R_{2}, \varphi\right\rangle:=\left\langle R_{2}, \omega^{s_{1}} \wedge \varphi\right\rangle+c \mathcal{U}_{s_{1}}\left(R_{1}, R_{2} \wedge \Omega^{+}\right)-c \mathcal{U}_{s_{1}}\left(R_{1}, R_{2} \wedge \Omega^{-}\right) .
$$

The right hand side of (19) is independent of the choice of $c, \Omega^{ \pm}$and depends linearly on $\varphi$. Moreover, $R_{1} \wedge R_{2}$ defines a positive closed $\left(s_{1}+s_{2}, s_{1}+s_{2}\right)$-current of mass 1 with support in $\operatorname{supp}\left(R_{1}\right) \cap \operatorname{supp}\left(R_{2}\right)$ which depends linearly on each $R_{i}$ and is 
symmetric with respect to the variables. The notion of wedgeability behave well with the notion of H-regularity:

Lemma A.1.14. - Let $R_{i}$ and $R_{i}^{\prime}$ be currents in $\mathscr{C}_{s_{i}}$. Assume that $R_{1}$ and $R_{2}$ are wedgeable. If $R_{i}^{\prime}$ is more $H$-regular than $R_{i}$ for $i=1,2$, then $R_{1}^{\prime}$ and $R_{2}^{\prime}$ are wedgeable and $R_{1}^{\prime} \wedge R_{2}^{\prime}$ is more $H$-regular than $R_{1} \wedge R_{2}$.

We will use the following proposition in the construction of the equilibrium measure:

Proposition A.1.15. - Let $R_{1}, R_{2}$ be wedgeable currents as above and $R_{i, n}$ be currents in $\mathscr{C}_{s_{i}}$ converging to $R_{i}$ in the Hartogs' sense. Then, $R_{1, n}, R_{2, n}$ are wedgeable and $R_{1, n} \wedge R_{2, n}$ converge to $R_{1} \wedge R_{2}$ in the Hartogs' sense.

For several currents (more than 2), the notion of wedgeability is defined by induction: that is $R_{1}, R_{2}$ and $R_{3}$ are wedgeable if $R_{1}$ and $R_{2}$ are wedgeable and $R_{1} \wedge R_{2}$ and $R_{3}$ are wedgeable. One shows that this definition is in fact symmetric in the $R_{i}$ and we have Proposition A.1.15 for several currents.

An interesting subcase is when we consider currents $R_{1}, \ldots, R_{l}$ such that $R_{i}$ is of bidegree $(1,1)$ for $i \geq 2$. For $2 \leq i \leq l$, there is a quasi-psh function $u_{i}$ on $\mathbb{P}^{k}$ such that

$$
d d^{c} u_{i}=R_{i}-\omega
$$

LEMmA A.1.16. - The currents $R_{1}, \ldots, R_{l}$ are wedgeable if and only if for every $2 \leq i \leq l, u_{i}$ is integrable with respect to the trace measure of $R_{1} \wedge \cdots \wedge R_{i-1}$. In particular, the last condition is symmetric with respect to $R_{2}, \ldots, R_{l}$.

If $R_{2}$ has a quasi-potential integrable with respect to $R_{1}$, it is classical to define the wedge-product $R_{1} \wedge R_{2}$ by

$$
R_{1} \wedge R_{2}:=d d^{c}\left(u_{2} R_{1}\right)+\omega \wedge R_{1} .
$$

One defines $R_{1} \wedge \cdots \wedge R_{l}$ by induction. These two definitions coincide.

The other use of super-potentials is to define pull-back and push-forward of current by meromorphic maps (see section 5.1 in [31]). We state the result in the case where $f$ is birational although the results are true in the case where $f$ is just meromorphic. Recall that pull-back and push-forward of a current are defined formally by formulae (3) and (4) of the previous section:

$$
\begin{aligned}
& f^{*}(S):=\left(\pi_{1}\right)_{*}\left(\pi_{2}^{*}(S) \wedge[\Gamma]\right) \\
& f_{*}(R):=\left(\pi_{2}\right)_{*}\left(\pi_{1}^{*}(R) \wedge[\Gamma]\right),
\end{aligned}
$$

where $[\Gamma]$ is the current of integration of $\Gamma$. We denote by $I^{+}:=I(f)$ and $I^{-}=$ $I^{\prime}(f)=I\left(f^{-1}\right)$ the indeterminacy sets of $f$ and $f^{-1}$. 
In particular, for a current in $R \in \mathscr{C}_{k-s+1}$ smooth near $I^{+}$the push-forward is a well defined positive closed $(k-s+1, k-s+1)$-current and the mass $\lambda_{s-1}$ of $f_{*}(R)$ does not depend on $R$. Similarly, for a current $S$ in $C_{s}$ smooth near $I^{-}$the pull-back is a well defined positive closed $(s, s)$-current and the mass of $f^{*}(S)$ is equal to $\lambda_{s}$. So as above we define for these currents $\Lambda(R)=\lambda_{s-1}^{-1} f_{*}(R)$ and $L(S)=\lambda_{s}^{-1} f^{*}(S)$ (the normalized push-forward and pull-back).

Using the theory of super-potentials we can extend these definitions to other currents. Namely, we say that a current $S \in \mathscr{C}_{s}$ is $f^{*}$-admissible if there exists a current $R_{0} \in \mathscr{C}_{k-s+1}$ which is smooth on a neighborhood of $I^{+}$such that the super-potentials of $S$ are finite at $\Lambda\left(R_{0}\right)$. For such $S$, if $\left(S_{n}\right)$ is a sequence of currents converging in the Hartogs' sense to $S$ then $S_{n}$ is $f^{*}$-admissible and $\left(\lambda_{s}\right)^{-1} f^{*}\left(S_{n}\right)$ converges in the Hartogs' sense to a limit independent on the choice of $\left(S_{n}\right)$ that we denote $\left(\lambda_{s}\right)^{-1} f^{*}(S)$ (in particular $f^{*}(S)$ is of mass $\lambda_{s}$ ). In other words, we have the continuity result:

Theorem A.1.17. - Let $S$ be an $f^{*}$ admissible current. Let $S_{n}$ be a sequence converging to $S$ in the Hartogs' sense, then $S_{n}$ is $f^{*}$-admissible and $L\left(S_{n}\right)$ converges in the Hartogs' sense to $L(S)$.

We say that $S$ is invariant under $f^{*}$ or that $S$ is $f^{*}$-invariant if $S$ is $f^{*}$-admissible and $L(S)=S$.

Proposition A.1.18. - Let $S$ be an $f^{*}$-admissible current in $\mathscr{C}_{s}$. Let $\mathcal{U}_{S}, \mathcal{U}_{L\left(\omega^{s}\right)}$ be super-potentials of $S$ and $L\left(\omega^{s}\right)$. Then $\lambda_{s}^{-1} \lambda_{s-1} \mathcal{U}_{S} \circ \Lambda+\mathcal{U}_{L\left(\omega^{s}\right)}$ is equal to a superpotential of $L(S)$ on $R \in \mathscr{C}_{k-s+1}$, smooth in a neighbourhood of $I^{+}$.

Similarly, one define push-forward of currents. We remark that an element $S \in$ $\mathscr{C}_{s}$ smooth near $I^{-}$is $f^{*}$-admissible and that the two available definitions of $L(S)$ coincide.

\section{A.2. Additional properties}

We state now some properties of the super-potentials that we need. Recall that $f \in \mathscr{B}$ and that $s$ is such that $\operatorname{dim}\left(I^{+}\right)=k-s-1$ and $\operatorname{dim}\left(I^{-}\right)=s-1$.

Lemma A.2.1. - Let $S_{1} \in \mathscr{C}_{r_{1}}$ and $S_{2} \in \mathscr{C}_{r_{2}}$ be wedgeable currents with $r_{1}+r_{2} \leq k$. There exist super-potentials $\mathcal{U}_{S_{1} \wedge S_{2}}, \mathcal{U}_{S_{1}}$ and $\mathcal{U}_{S_{2}}$ of $S_{1} \wedge S_{2}, S_{1}$ and $S_{2}$ such that:

$$
U_{S_{1} \wedge S_{2}}(R)=\mathcal{U}_{S_{1}}\left(R \wedge S_{2}\right)+\mathcal{U}_{S_{2}}\left(R \wedge \omega^{r_{1}}\right)
$$

for all $R \in \mathscr{C}_{k-r_{1}-r_{2}+1}$ such that $R$ and $S_{2}$ are wedgeable. 
Proof. - Let $S_{1, n}$ and $S_{2, m}$ be sequence of smooth currents in $\mathscr{C}_{r_{1}}$ and $\mathscr{C}_{r_{2}}$ converging to $S_{1}$ and $S_{2}$ in the Hartogs' sense. If $U_{S_{1, n}}$ and $U_{S_{2, m}}$ are smooth quasi-potentials of $S_{1, n}$ and $S_{2, m}$, then:

$$
S_{1, n} \wedge S_{2, m}=d d^{c}\left(U_{S_{1, n}} \wedge S_{2, m}+U_{S_{2, m}} \wedge \omega^{r_{1}}\right)+\omega^{r_{1}+r_{2}} .
$$

So, if $\mathcal{U}_{S_{1, n} \wedge S_{2, m}}, \mathcal{U}_{S_{1, n}}$ and $\mathcal{U}_{S_{2, m}}$ are super-potentials of mean 0 of the currents, we have for any $R$ in $\mathscr{C}_{k-r_{1}-r_{2}+1}$ :

$$
\mathcal{U}_{S_{1, n} \wedge S_{2, m}}(R)=\mathcal{U}_{S_{1, n}}\left(S_{2, m} \wedge R\right)+\mathcal{U}_{S_{2, m}}\left(\omega^{r_{1}} \wedge R\right)-\mathcal{U}_{S_{1, n}}\left(S_{2, m} \wedge \omega^{k-r_{1}-r_{2}+1}\right) .
$$

Now, we take $R$ such that $R$ and $S_{2}$ are wedgeable. We let $n \rightarrow \infty$. By Proposition A.1.15, $S_{1, n} \wedge S_{2, m}$ converges in the Hartogs' sense to $S_{1} \wedge S_{2, m}$. So by Proposition A.1.6, we have that:

$$
U_{S_{1} \wedge S_{2, m}}(R)=\mathcal{U}_{S_{1}}\left(S_{2, m} \wedge R\right)+\mathcal{U}_{S_{2, m}}\left(\omega^{r_{1}} \wedge R\right)-\mathcal{U}_{S_{1}}\left(S_{2, m} \wedge \omega^{k-r_{1}-r_{2}+1}\right),
$$

where the super-potentials are of mean 0 . Similarly, we let $m \rightarrow \infty$. Recall that $S_{2, m} \wedge R$ converges to $S_{2} \wedge R$ in the Hartogs' sense, hence $\mathcal{U}_{S_{1}}\left(S_{2, m} \wedge R\right)=\mathcal{U}_{S_{2, m} \wedge R}\left(S_{1}\right)$ converges to $\mathcal{U}_{S_{2} \wedge R}\left(S_{1}\right)$. So we have indeed:

$$
U_{S_{1} \wedge S_{2}}(R)=U_{S_{1}}\left(S_{2} \wedge R\right)+U_{S_{2}}\left(\omega^{r_{1}} \wedge R\right)-U_{S_{1}}\left(S_{2} \wedge \omega^{k-r_{1}-r_{2}+1}\right) .
$$

Since $U_{S_{1}}\left(S_{2} \wedge \omega^{k-r_{1}-r_{2}+1}\right)$ does not depend on $R$ and is finite because $S_{1}$ and $S_{2}$ are wedgeable, we can add it to $\mathcal{U}_{S_{1} \wedge S_{2}}$ (this just change the mean of the super-potentials) and we have the lemma.

Lemma A.2.2. - Let $T_{1} \in \mathscr{C}_{r_{1}}$ be an $f_{*}$-admissible current with $r_{1} \geq k-s$. Let $T_{2} \in \mathcal{C}_{r_{2}}$ be an $f^{*}$-admissible current with $r_{1}+r_{2} \leq k$ such that $L\left(T_{2}\right)$ and $T_{1}$ are wedgeable and $L\left(T_{2}\right) \wedge T_{1}$ is $f_{*}$-admissible. Assume also that $T_{2}$ and $\Lambda\left(T_{1}\right)$ are wedgeable. Then:

$$
\Lambda\left(L\left(T_{2}\right) \wedge T_{1}\right)=T_{2} \wedge \Lambda\left(T_{1}\right) .
$$

Proof. - Assume first that $T_{2}$ is smooth. Let $T_{1, n}$ and $L_{2, m}$ be sequences in $\mathscr{C}_{r_{1}}$ and $\mathscr{C}_{r_{2}}$ converging in the Hartogs' sense to $T_{1}$ and $L\left(T_{2}\right)$. Let $\Theta$ be a smooth current of bidegree $k-r_{1}-r_{2}$. We want to show that:

$$
\left\langle T_{2} \wedge \Lambda\left(T_{1}\right), \Theta\right\rangle=\left\langle\Lambda\left(L\left(T_{2}\right) \wedge T_{1}\right), \Theta\right\rangle
$$

for all $\Theta$ smooth.

First assume that $\Theta$ is closed and (strongly) positive. Up to a multiplicative constant, we assume that $\Theta \in \mathscr{C}_{k-r_{1}-r_{2}}$. Since everything is smooth:

$$
\begin{aligned}
\left\langle\Lambda\left(L_{2, m} \wedge T_{1, n}\right), \Theta\right\rangle & =\left\langle L_{2, m} \wedge T_{1, n}, L(\Theta)\right\rangle \\
& =\left\langle T_{1, n}, L_{2, m} \wedge L(\Theta)\right\rangle .
\end{aligned}
$$

Since $L_{2, m}$ converges to $L\left(T_{2}\right)$, we have that $L_{2, m} \wedge L(\Theta)$ converges to $L\left(T_{2} \wedge \Theta\right)$ in the sense of currents. Indeed, the sequence $\left(L_{2, m} \wedge L(\Theta)\right)_{m}$ is of mass 1 . We can 
extract a converging subsequence (in the sense of currents). Observe that its limit is less than $L\left(T_{2}\right) \wedge L\left(\omega^{k-r_{1}-r_{2}}\right)$ which gives no mass to $I^{+}$by dimension's arguments. So its limit gives no mass to $I^{+}$either. But outside $I^{+}, L_{2, m} \wedge L(\Theta)$ converges to the smooth form $L\left(T_{2}\right) \wedge L(\Theta)$. That implies that $L_{2, m} \wedge L(\Theta)$ converges to the trivial extension of $L\left(T_{2}\right) \wedge L(\Theta)$. This extension is equal to the form $L\left(T_{2} \wedge \Theta\right)$ since it has coefficients in $L^{1}$ (as in Lemma 2.1.1 test the convergence against a smooth form $\Psi$ and write it $\xi \Psi+(1-\xi) \Psi$ where $\xi$ is a cut-off function equal to 1 in a small neighborhood of $I^{+}$).

So, letting $m \rightarrow \infty$ and using the fact that $T_{2} \wedge \Theta$ is smooth give:

$$
\begin{aligned}
\lim _{m \rightarrow \infty}\left\langle\Lambda\left(L_{2, m} \wedge T_{1, n}\right), \Theta\right\rangle & =\left\langle T_{1, n}, L\left(T_{2} \wedge \Theta\right)\right\rangle \quad=\left\langle\Lambda\left(T_{1, n}\right), T_{2} \wedge \Theta\right\rangle \\
& =\left\langle T_{2} \wedge \Lambda\left(T_{1, n}\right), \Theta\right\rangle .
\end{aligned}
$$

Now, we let $n \rightarrow \infty, \Lambda\left(T_{1, n}\right)$ converges to $\Lambda\left(T_{1}\right)$ in the Hartogs' sense (Proposition A.1.17) hence Proposition A.1.15 gives that $T_{2} \wedge \Lambda\left(T_{1, n}\right)$ converges to $T_{2} \wedge \Lambda\left(T_{1}\right)$ in the sense of currents. On the other hand, when $n$ and $m$ go to $\infty, \Lambda\left(L_{2, m} \wedge T_{1, n}\right)$ converge to $\Lambda\left(L\left(T_{2}\right) \wedge T_{1}\right)$ in the sense of currents by Propositions A.1.15 and A.1.17.

So we have indeed that:

$$
\left\langle T_{2} \wedge \Lambda\left(T_{1}\right), \Theta\right\rangle=\left\langle\Lambda\left(L\left(T_{2}\right) \wedge T_{1}\right), \Theta\right\rangle
$$

for $\Theta$ closed.

Now, for $\Theta$ not necessarily closed, we can assume that $\Theta$ is positive and $\Theta \leq$ $C \omega^{k-r_{1}-r_{2}}$ for $C$ large enough. Again, we have that

$$
\left\langle\Lambda\left(L_{2, m} \wedge T_{1, n}\right), \Theta\right\rangle=\left\langle T_{1, n}, L_{2, m} \wedge L(\Theta)\right\rangle .
$$

The positive current $L_{2, m} \wedge L(\Theta)$ is less than $C L_{2, m} \wedge L\left(\omega^{k-r_{1}-r_{2}}\right)$ so it is of mass less than $C$. We can extract a converging subsequence (in the sense of currents). Observe that its limit is less than $C L\left(T_{2}\right) \wedge L\left(\omega^{k-r_{1}-r_{2}}\right)=C L\left(T_{2} \wedge \omega^{k-r_{1}-r_{2}}\right)$ which gives no mass to $I^{+}$by dimension's arguments. So its limit gives no mass to $I^{+}$either. Again outside $I^{+}, L_{2, m} \wedge L(\Theta)$ converges to the smooth form $L\left(T_{2}\right) \wedge L(\Theta)$. That implies that $L_{2, m} \wedge L(\Theta)$ converges to the trivial extension of $L\left(T_{2}\right) \wedge L(\Theta)$ which is equal to the form $L\left(T_{2} \wedge \Theta\right)$ which has coefficients in $L^{1}$. We have again that:

$$
\left\langle T_{1, n}, L\left(T_{2} \wedge \Theta\right)\right\rangle=\left\langle T_{2} \wedge \Lambda\left(T_{1, n}\right), \Theta\right\rangle \text {. }
$$

That gives the conclusion as before.

Now, for $T_{2}$ not necessarily smooth, we can approximate $T_{2}$ by a sequence of smooth currents converging in the Hartogs' sense to $T_{2}$. Since both members of the equality:

$$
\Lambda\left(L\left(T_{2}\right) \wedge T_{1}\right)=T_{2} \wedge \Lambda\left(T_{1}\right)
$$


depend continuously on $T_{2}$ for the Hartogs' convergence (wedge-product, pull-pack and push-forward are continuous for the Hartogs' convergence) we get the lemma from the smooth case.

Some of the hypothesis of the following lemma are not necessary, but the following version is enough for our purpose:

Lemma A.2.3. - Let $S_{1}, S_{2}$ and $S_{3}$ in $\mathscr{C}_{r_{1}}, \mathscr{C}_{r_{2}}$ and $\mathscr{C}_{r_{3}}$ with $r_{1}+r_{2}+r_{3}=k+$ 1. Assume that $S_{2}$ is smooth and that $L\left(S_{2}\right)$ and $S_{3}$ are wedgeable. Assume that $L\left(S_{2}\right) \wedge S_{3}$ is $f_{*}$-admissible. Assume also that the super-potential $\mathcal{U}_{S_{1}}$ of $S_{1}$ is finite at $\Lambda\left(L\left(S_{2}\right) \wedge S_{3}\right)$. Finally, we also assume that $S_{1}$ is $f^{*}$-admissible, that $S_{3}$ and $L\left(S_{1}\right)$ are wedgeable and that their wedge product is finite at the super-potential $\mathcal{U}_{L\left(S_{2}\right)}$ of $L\left(S_{2}\right)$. Then we have the formula:

$$
\begin{aligned}
\mathcal{U}_{S_{1}}\left(\Lambda\left(L\left(S_{2}\right) \wedge S_{3}\right)\right)= & \left(\frac{\lambda_{r_{1}}}{\lambda_{r_{1}-1}}\right)\left(\mathcal{U}_{L\left(S_{2}\right)}\left(S_{3} \wedge L\left(S_{1}\right)\right)-\mathcal{U}_{L\left(S_{2}\right)}\left(S_{3} \wedge L\left(\omega^{r_{1}}\right)\right)\right) \\
& +\mathcal{U}_{S_{1}}\left(\Lambda\left(\omega^{r_{2}} \wedge S_{3}\right)\right)
\end{aligned}
$$

Proof. - First, observe that $\omega^{r_{1}}$ is more H-regular than $S_{1}$ hence $L\left(\omega^{r_{1}}\right)$ is more Hregular than $L\left(S_{1}\right)$. So, we have that $S_{3}$ and $L\left(\omega^{r_{1}}\right)$ are wedgeable and $S_{3} \wedge L\left(\omega^{r_{1}}\right)$ is more H-regular than $S_{3} \wedge L\left(S_{1}\right)$. In particular, $\mathcal{U}_{L\left(S_{2}\right)}\left(S_{3} \wedge L\left(\omega^{r_{1}}\right)\right)$ is finite. Similarly, the expression $\mathcal{U}_{S_{1}}\left(\Lambda\left(\omega^{r_{2}} \wedge S_{3}\right)\right)$ is finite and everything is well defined in it.

Let $S_{1, m_{1}}, L_{2, m_{2}}$ and $S_{3, m_{3}}$ be sequences of smooth currents converging in the Hartogs' sense to $S_{1}, L\left(S_{2}\right)$ and $S_{3}$. Let $U_{1, m_{1}}$ and $U_{2, m_{2}}$ be smooth quasi-potential of $S_{1, m_{1}}$ and $L_{2, m_{2}}$. For smooth currents, we have the identity:

$$
\begin{aligned}
\mathcal{U}_{S_{1, m_{1}}}\left(\Lambda\left(L_{2, m_{2}} \wedge S_{3, m_{3}}\right)\right) & =\left\langle U_{1, m_{1}}, \Lambda\left(L_{2, m_{2}} \wedge S_{3, m_{3}}\right)\right\rangle \\
& =\left\langle\left(\lambda_{r_{1}-1}(f)\right)^{-1} f^{*}\left(U_{1, m_{1}}\right), L_{2, m_{2}} \wedge S_{3, m_{3}}\right\rangle .
\end{aligned}
$$

By Stokes, we recognize:

$$
\left\langle\left(\lambda_{r_{1}-1}(f)\right)^{-1} f^{*}\left(U_{1, m_{1}}\right), \omega^{r_{2}} \wedge S_{3, m_{3}}\right\rangle+\left\langle d d^{c}\left(\left(\lambda_{r_{1}-1}(f)\right)^{-1} f^{*}\left(U_{1, m_{1}}\right)\right), U_{2, m_{2}} \wedge S_{3, m_{3}}\right\rangle .
$$

Since $f^{*}$ commutes with $d d^{c}$, we have that

$$
\begin{aligned}
d d^{c}\left(\left(\lambda_{r_{1}-1}(f)\right)^{-1} f^{*}\left(U_{1, m_{1}}\right)\right) & =\left(\lambda_{r_{1}-1}(f)\right)^{-1} f^{*}\left(S_{1, m_{1}}-\omega^{r_{1}}\right) \\
& =\left(\frac{\lambda_{r_{1}}}{\lambda_{r_{1}-1}}\right)\left(L\left(S_{1, m_{1}}\right)-L\left(\omega^{r_{1}}\right)\right) .
\end{aligned}
$$

The lemma follows then by letting $m_{1}$ then $m_{2}$ then $m_{3}$ go to $\infty$ and using the continuity of the wedge product, the pull-back, push-forward and value at a point for the super-potential for the Hartogs' convergence.

We also have the following integration by parts lemma: 
LemmA A.2.4. - Let $S_{1}, S_{2}$ and $S_{3}$ in $\mathscr{C}_{r_{1}}, \mathscr{C}_{r_{2}}$ and $\mathscr{C}_{r_{3}}$ with $r_{1}+r_{2}+r_{3}=k+1$. Assume that the $S_{i}$ are two by two wedgeable. Then if $\mathcal{U}_{S_{1}}$ and $\mathcal{U}_{S_{2}}$ are super-potentials of $S_{1}$ and $S_{2}$ finite at $S_{2} \wedge S_{3}$ and $S_{1} \wedge S_{3}$ :

$$
\mathcal{U}_{S_{1}}\left(S_{2} \wedge S_{3}\right)-\mathcal{U}_{S_{1}}\left(\omega^{r_{2}} \wedge S_{3}\right)=\mathcal{U}_{S_{2}}\left(S_{1} \wedge S_{3}\right)-\mathcal{U}_{S_{2}}\left(\omega^{r_{1}} \wedge S_{3}\right)
$$

Proof. - First observe that $\omega^{r_{2}} \wedge S_{3}$ and $\omega^{r_{1}} \wedge S_{3}$ are more H-regular than $S_{2} \wedge S_{3}$ and $S_{1} \wedge S_{3}$ so every term is finite.

Now, if every term is smooth, we write $U_{S_{1}}$ and $U_{S_{2}}$ quasi-potentials of $S_{1}$ and $S_{2}$. By Stokes:

$$
\begin{aligned}
\mathcal{U}_{S_{1}}\left(S_{2} \wedge S_{3}\right)-\mathcal{U}_{S_{1}}\left(\omega^{r_{2}} \wedge S_{3}\right) & =\left\langle U_{S_{1}}, S_{2} \wedge S_{3}-\omega^{r_{2}} \wedge S_{3}\right\rangle=\left\langle U_{S_{1}}, d d^{c} U_{2} \wedge S_{3}\right\rangle \\
& =\left\langle d d^{c} U_{S_{1}}, U_{2} \wedge S_{3}\right\rangle=\left\langle S_{1}, U_{2} \wedge S_{3}\right\rangle-\left\langle\omega^{r_{1}}, U_{2} \wedge S_{3}\right\rangle \\
& =\mathcal{U}_{S_{2}}\left(S_{1} \wedge S_{3}\right)-\mathcal{U}_{S_{2}}\left(\omega^{r_{1}} \wedge S_{3}\right) .
\end{aligned}
$$

So the result follows in the general case by Hartogs' convergence.

We also have the following refinement of Lemma A.1.14 whose proof is similar:

LEMmA A.2.5. - Let $R_{1, n}$ and $R_{2, m}$ be sequence of currents in $\mathscr{C}_{p_{1}}$ and $\mathscr{C}_{p_{2}}$ converging in the Hartogs' sense to $R_{1}$ and $R_{2}$ which are wedgeable. Then there exists a constant $A_{n, m}>0$ such that

$$
\mathcal{U}_{R_{1, n} \wedge R_{2, m}} \geq \mathcal{U}_{R_{1} \wedge R_{2}}-A_{n, m}
$$

where the super-potentials are of mean 0 and where $A_{n, m}$ is uniformly bounded from above in $n$ and $m$ and is arbitrarily small for $n$ and $m$ large enough.

Proof. - By Lemma A.1.14, $R_{1, n}$ and $R_{2, m}$ are wedgeable.

The symbols $U$ and $\mathcal{U}$ below denote quasi-potentials and super-potentials of mean 0 . Assume first that all the terms are smooth. By hypothesis, there is a constant $a$ such that $\mathcal{U}_{R_{1, n}}+a \geq \mathcal{U}_{R_{1}}$ and $\mathcal{U}_{R_{2, m}}+a \geq \mathcal{U}_{R_{2}}$. Write $r=k-p_{1}-p_{2}+1$. Consider a smooth form $R$ in $\mathscr{C}_{r}$ and choose $U_{R}$ smooth. We have the computation:

$$
\begin{aligned}
\mathcal{U}_{R_{1, n} \wedge R_{2, m}}(R) & =\left\langle R_{1, n} \wedge R_{2, m}, U_{R}\right\rangle=\left\langle R_{2, m}, \omega^{p_{1}} \wedge U_{R}\right\rangle+\left\langle R_{1, n}-\omega^{p_{1}}, R_{2, m} \wedge U_{R}\right\rangle \\
& =\left\langle R_{2, m}, \omega^{p_{1}} \wedge U_{R}\right\rangle+\left\langle d d^{c} U_{R_{1, n}}, R_{2, m} \wedge U_{R}\right\rangle \\
& =\left\langle R_{2, m}, \omega^{p_{1}} \wedge U_{R}\right\rangle+\left\langle U_{R_{1, n}}, R_{2, m} \wedge d d^{c} U_{R}\right\rangle \\
& =\left\langle R_{2, m}, \omega^{p_{1}} \wedge U_{R}\right\rangle+U_{R_{1, n}}\left(R_{2, m} \wedge R\right)-U_{R_{1, n}}\left(R_{2, m} \wedge \omega^{r}\right) . \\
& =U_{R}\left(R_{2, m} \wedge \omega^{p_{1}}\right)+U_{R_{1, n}}\left(R_{2, m} \wedge R\right)-U_{R_{1, n}}\left(R_{2, m} \wedge \omega^{r}\right) .
\end{aligned}
$$


That identity also holds when the currents are not smooth by Hartogs' convergence. We have the same identity for $R_{1} \wedge R_{2, m}$ and $R_{1} \wedge R_{2}$. By difference, we have:

$$
\begin{array}{r}
U_{R_{1, n} \wedge R_{2, m}}(R)-\mathcal{U}_{R_{1} \wedge R_{2, m}}(R)+\mathcal{U}_{R_{1} \wedge R_{2, m}}(R)-\mathcal{U}_{R_{1} \wedge R_{2}}(R)= \\
\mathcal{U}_{R_{1, n}}\left(R_{2, m} \wedge R\right)-\mathcal{U}_{R_{1}}\left(R_{2, m} \wedge R\right)-\mathcal{U}_{R_{1, n}}\left(R_{2, m} \wedge \omega^{r}\right)+\mathcal{U}_{R_{1}}\left(R_{2, m} \wedge \omega^{r}\right) \\
+\mathcal{U}_{R_{2, m}}\left(R_{1} \wedge R\right)-\mathcal{U}_{R_{2}}\left(R_{1} \wedge R\right)-\mathcal{U}_{R_{2, m}}\left(R_{1} \wedge \omega^{r}\right)+\mathcal{U}_{R_{2}}\left(R_{1} \wedge \omega^{r}\right) .
\end{array}
$$

So:

$$
\begin{array}{r}
U_{R_{1, n} \wedge R_{2, m}}(R)-U_{R_{1} \wedge R_{2}}(R) \geq \\
-2 a-\mathcal{U}_{R_{1, n}}\left(R_{2, m} \wedge \omega^{r}\right)+\mathcal{U}_{R_{1}}\left(R_{2, m} \wedge \omega^{r}\right)-U_{R_{2, m}}\left(R_{1} \wedge \omega^{r}\right)+U_{R_{2}}\left(R_{1} \wedge \omega^{r}\right) .
\end{array}
$$

The last quantity does not depend on $R$ and is uniformly bounded from below: the terms with a minus sign are greater than $-M$ since the super-potentials are of mean 0 , and since $R_{2, m} \wedge \omega^{r}$ converges to $R_{2} \wedge \omega^{r}$ in the Hartogs' sense and $U_{R_{1}}\left(R_{2} \wedge \omega^{r}\right)$ is finite, we have that $\mathcal{U}_{R_{1}}\left(R_{2, m} \wedge \omega^{r}\right)$ and $\mathcal{U}_{R_{2}}\left(R_{1} \wedge \omega^{r}\right)$ are uniformly bounded from below.

This gives that the constant $A_{n, m}$ of the lemma is uniformly bounded from above in $n$ and $m$. Now, we can choose $A_{n, m}$ going to zero by Proposition A.1.15: if not, we can extract subsequences such that $A_{n_{i}, m_{i}} \geq \varepsilon>0$ and it contradicts the fact that $R_{1, n_{i}} \wedge R_{2, m_{i}}$ converges in the Hartogs' sense to $R_{1} \wedge R_{2}$. 



\section{BIBLIOGRAPHY}

[1] E. Bedford \& J. Diller - "Energy and invariant measures for birational surface maps", Duke Math. J. 128 (2005), p. 331-368.

[2] E. Bedford, M. Lyubich \& J. Smillie - "Polynomial diffeomorphisms of $\mathbf{C}^{2}$. IV. The measure of maximal entropy and laminar currents", Invent. Math. 112 (1993), p. 77-125.

[3] E. Bedford \& J. Smillie - "Polynomial diffeomorphisms of $\mathbf{C}^{2}$ : currents, equilibrium measure and hyperbolicity", Invent. Math. 103 (1991), p. 69-99.

[4] _ "Polynomial diffeomorphisms of $\mathbf{C}^{2}$. III. Ergodicity, exponents and entropy of the equilibrium measure", Math. Ann. 294 (1992), p. 395-420.

[5] J.-Y. BRIEnd \& J. Duval - "Deux caractérisations de la mesure d'équilibre d'un endomorphisme de $\mathrm{P}^{k}(\mathbf{C})$ ", Publ. Math. Inst. Hautes Études Sci. 93 (2001), p. 145-159.

[6] D. Burguet - "A proof of Yomdin-Gromov's algebraic lemma", Israel J. Math. 168 (2008), p. 291-316.

[7] S. Cantat - "Dynamique des automorphismes des surfaces K3", Acta Math. 187 (2001), p. 1-57.

[8] E. M. ChIRKA - Complex analytic sets, Mathematics and its Applications (Soviet Series), vol. 46, Kluwer Academic Publishers Group, 1989.

[9] D. Coman \& V. Guedj - "Invariant currents and dynamical Lelong numbers", J. Geom. Anal. 14 (2004), p. 199-213.

[10] H. De ThÉLin - "Sur la construction de mesures selles", Ann. Inst. Fourier (Grenoble) 56 (2006), p. 337-372.

[11] _ "Sur les exposants de Lyapounov des applications méromorphes", Invent. Math. 172 (2008), p. 89-116.

[12] _ "Ahlfors' currents in higher dimension", in appear in Ann. Fac. Sci. Toulouse. 
[13] J.-P. Demailly - "Monge-Ampère operators, Lelong numbers and intersection theory", in Complex analysis and geometry, Univ. Ser. Math., Plenum, 1993, p. $115-193$.

[14] _ "Complex analytic and algebraic geometry", http://www-fourier. ujf-grenoble.fr/ demailly/books.html, 1997.

[15] J. Diller - "Dynamics of birational maps of $\mathbf{P}^{2}$ ", Indiana Univ. Math. J. 45 (1996), p. $721-772$.

[16] J. Diller, R. Dujardin \& V. Guedj - "Dynamics of meromorphic maps with small topological degree I: from cohomology to currents", preprint arXiv:0803.0955, to appear in Indiana Univ. Math. J.

[17] _ _ "Dynamics of meromorphic maps with small topological degree II: Energy and invariant measure", preprint arXiv:0805.3842, to appear in Comment. Math. Helvet.

[18] __ , "Dynamics of meromorphic maps with small topological degree III: geometric currents and ergodic theory", preprint arXiv:0806.0146, to appear in Ann. Ecole Norm. Sup.

[19] J. Diller \& V. Guedj - "Regularity of dynamical Green's functions", Trans. Amer. Math. Soc. 361 (2009), p. 4783-4805.

[20] T.-C. Dinh \& C. Dupont - "Dimension de la mesure d'équilibre d'applications méromorphes", J. Geom. Anal. 14 (2004), p. 613-627.

[21] T.-C. Dinh \& N. Sibony - "Dynamique des applications d'allure polynomiale", J. Math. Pures Appl. 82 (2003), p. 367-423.

[22] _ _Regularization of currents and entropy", Ann. Sci. École Norm. Sup. 37 (2004), p. 959-971.

[23] _ _ "Dynamics of regular birational maps in $\mathbb{P}^{k}$ ", J. Funct. Anal. 222 (2005), p. $202-216$.

[24] _ "Green currents for holomorphic automorphisms of compact Kähler manifolds", J. Amer. Math. Soc. 18 (2005), p. 291-312.

[25] _ _ "Une borne supérieure pour l'entropie topologique d'une application rationnelle", Ann. of Math. 161 (2005), p. 1637-1644.

[26] _ "Decay of correlations and the central limit theorem for meromorphic maps", Comm. Pure Appl. Math. 59 (2006), p. 754-768.

[27] _ _ "Distribution des valeurs de transformations méromorphes et applications", Comment. Math. Helv. 81 (2006), p. 221-258. 
[28] _ "Geometry of currents, intersection theory and dynamics of horizontallike maps", Ann. Inst. Fourier (Grenoble) 56 (2006), p. 423-457.

[29] _ "Pull-back currents by holomorphic maps", Manuscripta Math. 123 (2007), p. 357-371.

[30] - "Equidistribution towards the Green current for holomorphic maps", Ann. Sci. Éc. Norm. Supér. 41 (2008), p. 307-336.

[31] _ "Super-potentials of positive closed currents, intersection theory and dynamics", Acta Math. 203 (2009), p. 1-82.

[32] _ _ "Super-potentials for currents on compact Kähler manifolds and dynamics of automorphisms", preprint arXiv:0804.0860.

[33] R. DujARdin - "Laminar currents and birational dynamics", Duke Math. J. 131 (2006), p. 219-247.

[34] C. Favre - "Points périodiques d'applications birationnelles de $\mathbf{P}^{2} "$, Ann. Inst. Fourier (Grenoble) 48 (1998), p. 999-1023.

[35] C. Favre \& M. Jonsson - "Brolin's theorem for curves in two complex dimensions", Ann. Inst. Fourier (Grenoble) 53 (2003), p. 1461-1501.

[36] J. E. Fornæss \& N. Sibony - "Complex dynamics in higher dimension. I", Astérisque 222 (1994), p. 201-231.

[37] _ "Complex dynamics in higher dimensions", in Complex potential theory (Montreal, PQ, 1993), NATO Adv. Sci. Inst. Ser. C Math. Phys. Sci., vol. 439, Kluwer Acad. Publ., 1994, p. 131-186.

[38] J. E. Fornaess \& N. Sibony - "Complex dynamics in higher dimension. II", in Modern methods in complex analysis (Princeton, NJ, 1992), Ann. of Math. Stud., vol. 137, Princeton Univ. Press, 1995, p. 135-182.

[39] M. Gromov - "Entropy, homology and semialgebraic geometry", Astérisque 145-146 (1987), p. 225-240, Séminaire Bourbaki, vol. 1985/86, exposé nº 663.

[40] _ "Convex sets and Kähler manifolds", in Advances in differential geometry and topology, World Sci. Publ., Teaneck, NJ, 1990, p. 1-38.

[41] _ "On the entropy of holomorphic maps", Enseign. Math. 49 (2003), p. $217-235$.

[42] V. Guedj - "Courants extrémaux et dynamique complexe", Ann. Sci. École Norm. Sup. 38 (2005), p. 407-426.

[43] _ "Entropie topologique des applications méromorphes", Ergodic Theory Dynam. Systems 25 (2005), p. 1847-1855. 
[44] V. Guedu \& N. Sibony - "Dynamics of polynomial automorphisms of $\mathbf{C}^{k}$ ", Ark. Mat. 40 (2002), p. 207-243.

[45] A. KATOK \& B. HASSELBlatT - Introduction to the modern theory of dynamical systems, Encyclopedia of Mathematics and its Applications, vol. 54, Cambridge Univ. Press, 1995.

[46] S. ŁoJASIEWICZ - Introduction to complex analytic geometry, Birkhäuser, 1991.

[47] R. MañÉ - "A proof of Pesin's formula", Ergodic Theory Dynam. Systems 1 (1981), p. $95-102$.

[48] A. Russakovskit \& B. Shiffman - "Value distribution for sequences of rational mappings and complex dynamics", Indiana Univ. Math. J. 46 (1997), p. 897-932.

[49] N. Sibony - "Quelques problèmes de prolongement de courants en analyse complexe", Duke Math. J. 52 (1985), p. 157-197.

[50] _ _ "Dynamique des applications rationnelles de $\mathbf{P}$ ", in Dynamique et géométrie complexes (Lyon, 1997), Panoramas \& Synthèses, vol. 8, Soc. Math. France, 1999.

[51] Y. T. SiU - "Analyticity of sets associated to Lelong numbers and the extension of closed positive currents", Invent. Math. 27 (1974), p. 53-156.

[52] H. SKoda - "Prolongement des courants, positifs, fermés de masse finie", Invent. Math. 66 (1982), p. 361-376.

[53] G. VignY - "Dirichlet-like space and capacity in complex analysis in several variables", J. Funct. Anal. 252 (2007), p. 247-277.

[54] P. Walters - An introduction to ergodic theory, Graduate Texts in Math., vol. 79, Springer, 1982.

[55] Y. Yomdin - "Volume growth and entropy", Israel J. Math. 57 (1987), p. 285300 . 